\title{
A Work-Pattern Centric Approach to Building a Personal Knowledge Advantage Machine
}

\author{
Daniel Sloan \\ West Virginia University
}

Follow this and additional works at: https://researchrepository.wvu.edu/etd

\section{Recommended Citation}

Sloan, Daniel, "A Work-Pattern Centric Approach to Building a Personal Knowledge Advantage Machine" (2012). Graduate Theses, Dissertations, and Problem Reports. 4919.

https://researchrepository.wvu.edu/etd/4919

This Thesis is protected by copyright and/or related rights. It has been brought to you by the The Research Repository @ WVU with permission from the rights-holder(s). You are free to use this Thesis in any way that is permitted by the copyright and related rights legislation that applies to your use. For other uses you must obtain permission from the rights-holder(s) directly, unless additional rights are indicated by a Creative Commons license in the record and/ or on the work itself. This Thesis has been accepted for inclusion in WVU Graduate Theses, Dissertations, and Problem Reports collection by an authorized administrator of The Research Repository @ WVU. For more information, please contact researchrepository@mail.wvu.edu. 


\title{
A Work-Pattern Centric Approach to Building a Personal Knowledge Advantage Machine
}

\author{
Daniel Sloan
}

Thesis submitted to the

College of Engineering and Mineral Resources

at West Virginia University

in partial fulfillment of the requirements

for the degree of

Master of Science

in

Computer Science

Yenumula V. Reddy, Ph.D., Chair

Bojan Cukic, Ph.D.

Cynthia D. Tanner, MS.

Lane Department of Computer Science and Electrical Engineering

Morgantown, West Virginia

2012

Keywords: Work-patterns, file usage, semantic desktop, machine learning

Copyright (C) 2012 Daniel Sloan 


\begin{abstract}
A Work-Pattern Centric Approach to Building a Personal Knowledge Advantage Machine Daniel Sloan
\end{abstract}

A work pattern, also known as a usage pattern, can be broadly defined as the methods by which a user typically utilizes a particular system. Data mining has been applied to web usage patterns for a variety of purposes. This thesis presents a framework by which data mining techniques could be used to extract patterns from an individual's work flow data in order facilitate a new type of architecture known as a knowledge advantage machine. This knowledge advantage machine is a type of semantic desktop and semantic web application that would assist people in constructing their own personal knowledge networks, as well as sharing that information in an efficient manner with colleagues using the same system. A knowledge advantage machine would be capable of automatically discovering new knowledge which is relevant to the user's personal ontology.

Through experimentation, we demonstrate that a user's file usage patterns can be utilized by software in order to automatically and seamlessly learn what is "important" as defined by the user. Further research is necessary to apply this principle to a more realized knowledge advantage machine such that decisions can be fueled by work patterns as well as semantic or contextual information. 


\section{Dedication}

I dedicate this work to my grandfather.

Spoczywaj w pokoju. 


\section{Acknowledgments}

I would like to thank my colleagues and friends who have inspired me, motivated me, and overall have been there for me even when things seemed bleak. To those who have inspired my best and weathered my worst: Bryan Lemon, Jonathan Lynch, Brian Cain, Alex Dauphin, Lisa Soros.

I would also like to thank Dr. Reddy, without whom this thesis would not have been possible. He has taught me the importance of lateral thinking, especially when dealing with such complex and pervasive problems as the ones described in this work.

Thanks are given to all of my wonderful volunteers, who gave up their valuable time and hard drive space to help me obtain the empirical data that drives this thesis.

I give thanks to those instructors that I've worked under in the capacity of teaching assistantship: Mrs. Tanner, Mrs. Hayhurst, and, again, Dr. Reddy. I have learned as much in the process of teaching computer science as I did in my time as an undergraduate.

I must thank all of the truly excellent professors I have had the pleasure of learning from throughout my years at WVU. The professionalism, character, and strength of personality within the Lane Department of Computer Science and Electrical Engineering cannot possibly be overstated.

Finally, I thank Dr. Katerina Goseva-Popstojanova, whose Empirical Methods class provided me with the extra kick I needed to get this thing done. 


\section{Contents}

1 Introduction 1

1.1 Problem Statement $\ldots \ldots \ldots \ldots$. . . . . . . . . . . . . . . 3

1.1 .1 Sample Scenario . . . . . . . . . . . . . . . . . . . 3

1.2 Knowledge Advantage Machine . . . . . . . . . . . . . . . . . 5

$1.3 \quad$ KAM Architectural View $\ldots \ldots \ldots \ldots$

1.4 Contributions of Thesis $\ldots \ldots \ldots \ldots \ldots$

1.5 Outline of Thesis $\ldots \ldots \ldots \ldots \ldots \ldots$

\begin{tabular}{|lll}
2 & Research Objectives & 11
\end{tabular}

$2.1 \quad$ Method of Inquiry $\ldots \ldots \ldots \ldots$

2.2 Research Questions $\ldots \ldots \ldots \ldots$

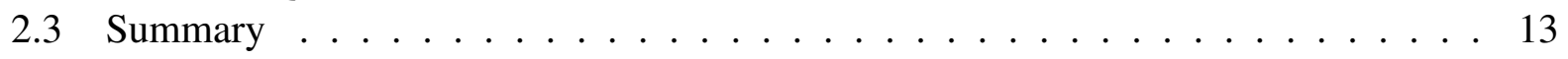

$\begin{array}{llr}3 & \text { Background and Related Work } & 15\end{array}$

3.1 Semantic Web . . . . . . . . . . . . . . . . . . . . . 16

3.2 Semantic Desktop . . . . . . . . . . . . . . . . . . . . . . . . . . 17

3.3 Web Usage Mining . . . . . . . . . . . . . . . . . . . . . . . . . . 17

3.3 .1 Preprocessing . . . . . . . . . . . . . . . . . . . . . 18

$3.3 .2 \quad$ Pattern Discovery.$\ldots \ldots \ldots$

3.3 .3 Pattern Analysis $\ldots \ldots \ldots$. . . . . . . . . . . . . . . . 20

3.4 Graph Based Induction $\ldots \ldots \ldots \ldots$

3.5 Ontological Research . . . . . . . . . . . . . . . . . . . . . . . 22

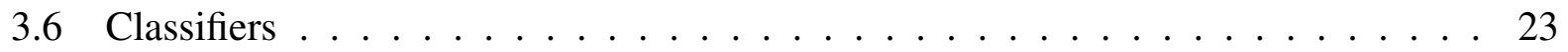

$3.6 .1 \quad$ Naive Bayes . . . . . . . . . . . . . . . . . . . . . 24

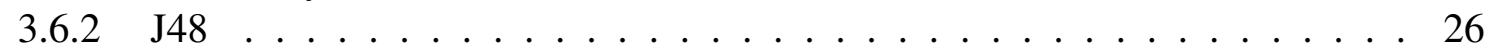

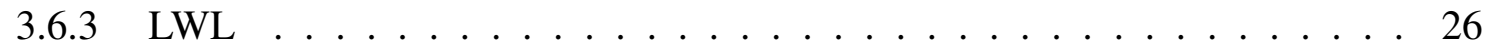

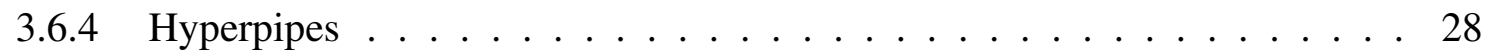

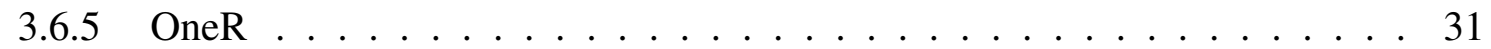

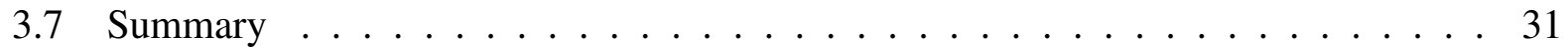

4 Work Pattern Centric Knowledge Advantage Machine 33

4.1 WPCKAM Architectural View $\ldots \ldots \ldots \ldots$. . . . . . . . . . . . . . 34

4.2 File Usage Prediction . . . . . . . . . . . . . . . . . . . . . . . . . . . . 34 


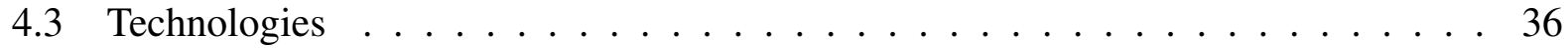

4.3 .1 Windows Auditing $\ldots \ldots \ldots \ldots$

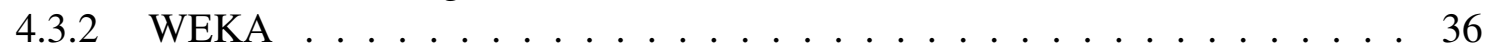

4.4 How the Advantage is Gained $\ldots \ldots \ldots$

4.4 .1 Context Awareness . . . . . . . . . . . . . . . . . . . . . . . 37

$4.4 .2 \quad$ Presenting Predicted Files . . . . . . . . . . . . . . . . . . 38

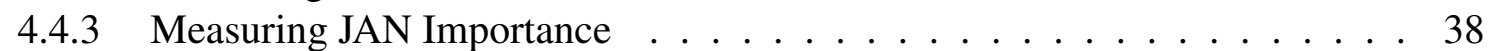

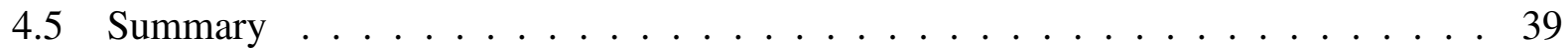

5 Experimental Setup and Analysis of Results 4

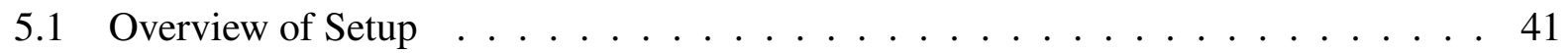

5.2 Preprocessing . . . . . . . . . . . . . . . . . . . 41

5.3 Results . . . . . . . . . . . . . . . . . . . . . . . . 42

5.4 Classification $\ldots \ldots \ldots \ldots \ldots \ldots$. . . . . . . . . . . . . . . . . . . 48

$5.4 .1 \quad$ Hyperpipes $\ldots \ldots \ldots \ldots \ldots$. . . . . . . . . . . . . . . 48

5.4 .2 Naive Bayes Classification . . . . . . . . . . . . . . . . . . . 48

5.4 .3 J48 Classification . . . . . . . . . . . . . . . . . . . . . . . . . . . . . . 48

$5.4 .4 \quad$ LWL Classification . . . . . . . . . . . . . . . . . . . . . . . . . . . . 49

5.4 .5 OneR Classification . . . . . . . . . . . . . . . . . . . . . . . . 49

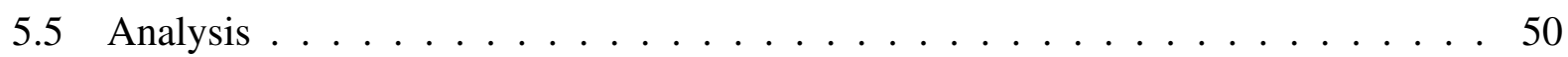

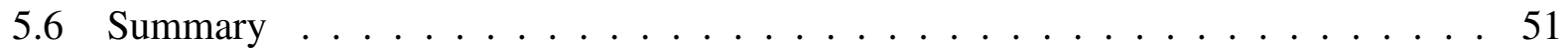

6 Conclusions

6.1 Hypotheses . . . . . . . . . . . . . . . . . . . . . . 52

6.2 How the Advantage is Gained $\ldots \ldots \ldots \ldots$. . . . . . . . . . . 53

6.2 .1 Context Awareness . . . . . . . . . . . . . . . . . . . . . . . 54

6.2 .2 Presenting Predicted Files $\ldots \ldots \ldots \ldots \ldots \ldots$

6.2 .3 Measuring JAN Importance $\ldots \ldots \ldots \ldots$

6.3 Future Work . . . . . . . . . . . . . . . . . . 55

\begin{tabular}{|l|l} 
A Reproducing the Experiments & 56
\end{tabular}

A.1 Acquiring the Software $\ldots \ldots \ldots \ldots \ldots \ldots$

A.2 Obtaining Data $\ldots \ldots \ldots \ldots \ldots \ldots \ldots$

A.3 Running your Experiments $\ldots \ldots \ldots \ldots \ldots \ldots$

B Raw Data and Charts 59

B.1 Various Charts . . . . . . . . . . . . . . . . . . . . . . . . . 59 


\section{List of Figures}

1.1 KAM Objective Map . . . . . . . . . . . . . . . . . . . . . . 6

1.2 Vijjana Architecture $[30] \ldots \ldots \ldots \ldots$

1.3 KAM Architecture $\ldots \ldots \ldots \ldots \ldots \ldots$

$3.1 \quad$ Pairwise Chunking [26] . . . . . . . . . . . . . . . . . . . . 22

3.2 Pseudo code for Naive Bayes $[20]$. . . . . . . . . . . . . . . . . 25

3.3 Symmetry Principle for Probability Ratios [17] . . . . . . . . . . . . . . . . . 25

3.4 Naive Bayes Classifier [44] $\ldots \ldots \ldots \ldots \ldots$

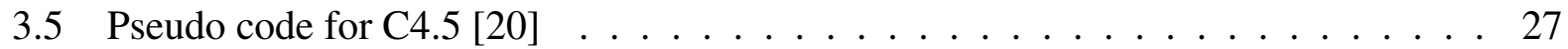

$3.6 \quad$ Pseudo code for K-Means $[18] \ldots \ldots \ldots \ldots$

$3.7 \quad$ Pseudo code for Locally Weighted Naive Bayes [20] . . . . . . . . . . . . . . . . 29

$3.8 \quad$ Pseudo code for Hyperpipes $[11] \ldots \ldots \ldots \ldots \ldots$

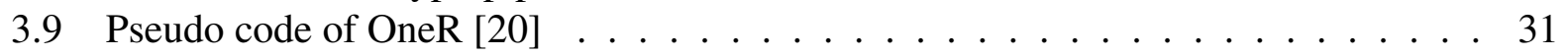

4.1 WPCKAM Architecture $\ldots \ldots \ldots$. . . . . . . . . . . . . . . . . . . . 34

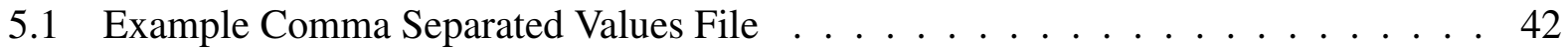




\section{List of Tables}

$5.1 \quad$ Prediction success rates by k-value for all datasets . . . . . . . . . . . . . . . . . 44

5.2 Prediction success rates by classifier for all datasets $\ldots \ldots \ldots$. . . . . . . . . 44

5.3 Prediction success rates by dataset for all datasets $\ldots \ldots \ldots$. . . . . . . . . . 44

5.4 Filtering success rates by $\mathrm{k}$-value for all datasets $\ldots \ldots \ldots$. . . . . . . . . . . 45

5.5 Filtering success rates by classifier for all datasets $\ldots \ldots \ldots$. . . . . . . . . 45

$5.6 \quad$ Filtering success rates by dataset for all datasets $\ldots \ldots \ldots$. . . . . . . . . 45

5.7 Prediction data with number of predictions by k-value for all datasets. . . . . . . . 46

5.8 Prediction data with number of predictions by classifier for all datasets . . . . . . . 46

5.9 Prediction data with number of predictions by dataset for all datasets $\ldots . . . . .446$

5.10 Filtering data with number of predictions by k-value for all datasets . . . . . . . 47

5.11 Filtering data with number of predictions by classifier for all datasets . . . . . . . . 47

5.12 Filtering data with number of predictions by dataset for all datasets . . . . . . . . . 47

B.2 Prediction success rates by all factors for all datasets . . . . . . . . . . . . . . 59

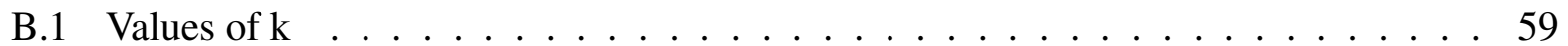

B.3 Filtering success rates by all factors for all datasets $\ldots \ldots \ldots$. . . . . . . . 73

B.4 List of document file extensions allowed through preprocessing whitelist . . . . . . 87

B.5 List of programming source code file extensions allowed through preprocessing

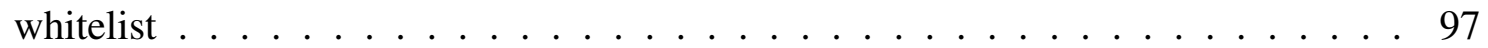

B.6 Record counts across datasets . . . . . . . . . . . . . . . . . . . . 107 


\section{Chapter 1}

\section{Introduction}

Knowledge workers' jobs continue to increase in both their complexity and their plenitude of associated information; this is sometimes referred to as "information overload" [3] , and is a well known problem, especially in technical fields. Therefore, a need for a mechanism capable of semantically organizing that information is apparent. In order to combat this problem we must develop a technology which we will refer to as a Knowledge Advantage Machine.

The "knowledge advantage" of a KAM comes from the similar but better-known idea of a "mechanical advantage," [9] wherein the force one uses to accomplish some physical task is reduced through the clever use of some mechanism or tool. A KAM would utilize a semantic link network [45] in order to better organize and utilize information for a given knowledge worker. Such a system would probably require some initial work on the user's part [40] to set up, but the goal would be that the resulting "knowledge advantage" would counteract this effort.

A KAM would consist of several functional parts, referred to as "agents." Agents are simply modules which act to perform some task; for a KAM, the most important agents are called Selection, Organization, Context Map, Display, and Collaboration. An envisioning of a KAM consisting of these five major agents is detailed in depth in $\$ 1.2$.

The necessary building blocks of a Knowledge Advantage Machine exist in an emerging field; 
for example, the semantic link networking model which works to establish semantic links across a variety of resources and is meant to be used as a semantic representation model for the Semantic Web, Semantic Grid, and the Knowledge Grid [45]. Long before this, however, the concept of an ontology itself was examined, such as in Gruber's 1995 work wherein methods of representing an ontology were explored [13].

A semantic link network, in its semantic organization of information for knowledge workers, would allow knowledge workers to perform their jobs with added ease and efficiency [40]. It follows logically that any properly designed Knowledge Advantage Machine would, in theory, facilitate the semantic link network in a manner which is more comfortable for knowledge workers to use than the traditional methods of information gathering and application.

The idea of monitoring usage patterns has long been used for websites, especially commerce sites, in order to present a more user-tailored experience [38]. File usage has also been recorded and statistically analyzed [39], but little has been done in the vein of examining or exploiting these file usage patterns for the sake of a knowledge advantage.

To this end, the purpose of this thesis is to present a framework for a KAM system which focuses upon desktop usage patterns in order to intuit ontological importance. This framework is constructed in order to demonstrate one possible basis for a knowledge advantage machine's ability to accelerate and enrich the daily tasks of any general knowledge worker, while providing an overview of the current state-of-the-art research into the various "building blocks" of the KAM.

Most specifically, this thesis offers a work pattern recognition tool which is capable of monitoring and recording file usage data, and make predictions about future file usage. I will empirically show the amount of accuracy that these classification learning based predictions offer. 


\subsection{Problem Statement}

The problem addressed by this thesis can be expressed as follows: "How can work-pattern centric agents augment the KAM's ability to provide its eponymous knowledge advantage?" If accurate, these predictions could be used to assist in the identification of context. Because the KAM is predicated upon being able to determine needed JANS $\Omega^{1}$ for a given context, alternative methods of context identification could prove very useful.

The problem that this thesis addresses is not that of creating a generic knowledge advantage machine, though the concept will be explored and a case will be made for its importance. Instead, the problem requires us to specifically explore the data collection and pattern discovery agents of a KAM which is capable of functioning partially or wholly based on work patterns. This thesis therefore addresses the problem of implementing and testing what we refer to as a "Work-Pattern Centric Approach to Building a Personal Knowledge Advantage Machine" with the ultimate main goal of improving context prediction.

\subsubsection{Sample Scenario}

It is perhaps easiest to explan the concept of a knowledge advantage machine by presenting a scenario in which it assists an average knowledge worker. Let us take the example of a professor, "Dr. Doe." Like most professors, Dr. Doe does research, but he also teaches. These categories - teaching and researching - would constitute two high-level "contexts" of his overall knowledge base. Within the context of research, Dr. Doe might be examining several different papers of varying topics such as nanotechnology or microprocessors; these would also constitute contexts, or, alternately, sub-contexts.

A fully idealized knowledge advantage machine would be aware of these contexts at any given time. This awareness could be achieved in a variety of ways; for the purposes of this example, we

\footnotetext{
${ }^{1}$ See $\$ 1.2$
} 
assume it exists and is more or less accurate. Therefore, if Dr. Doe was currently using his personal computer to perform research into nanotechnology, he would be considered to be currently in the "research" context. While these contexts represent states, they are not mutually exclusive; for example, a person can be doing research and writing a syllabus at the same time. Ideally, contexts could be tracked on-the-go, perhaps via mobile-devices, as well; Dr. Doe's personal KAM would be aware of his presence at a nanotechnology research meeting, therefore identifying him as currently residing in both the research and nanotechnology contexts. This idea is called "context aware computing" [5] and while it is true that several context-aware applications have been been built as demonstrations, mass-availability of this sort of technology is currently rare. Some exceptions do exist, such as mobile phone applications which alter phone behavior based on location [23].

Because Dr. Doe's KAM is constantly aware of his context, and the context of documents within his personal ontology, it is able to to perform several useful tasks. When Dr. Doe sits down to write a paper concerning nanotechnology, his KAM is constantly performing semantic analysis on his emerging document and concurrently searching for papers most related to that specific content. When Dr. Doe receives an email from a student requesting a syllabus for a particular class, a fully realized KAM would be "smart" enough to send the appropriate reply. This is because the KAM would, after examining the email, be privy to several facts; most importantly, the sender's email address identifies the sender as a student in a particular class, and the semantic content of the email points to the user requesting a syllabus document.

By combining context aware computing with a semantically understood personal ontology, a KAM would be able to perform many such time-saving knowledge-intensive tasks. However, this is a complicated and vast problem, so the purpose of this thesis is limited to examining one possible way in which context could be determined. Under the system detailed in this research, Dr. Doe's context would be identified by his patterns of file access. With accurate file usage prediction, Dr. Doe might also be offered a pane of files that he is likely to access in the near future. 


\subsection{Knowledge Advantage Machine}

The concept of a Knowledge Advantage Machine is a combination of several other discrete ideas. In 2011 Luyi Wang et al. [40] published a context-centric approach to creating a knowledge advantage machine. This paper provides a basis for the problem; a KAM can be succinctly defined as any device which increases the efficiency at which a knowledge worker performs daily tasks.

Likewise, we aim to reduce the force exerted by a knowledge worker to accomplish tasks in their fields. Applications of a KAM are broad: a chemical engineer's KAM might automatically discover exciting new research papers in the field of chemistry and insert them into his ontology for perusal, while an instructor's KAM might automatically prepare draft email replies to students who have sent requests for syllabi or assignments. A fully realized KAM would allow for increased collaboration; for example, two computer scientists might share their ontological knowledge bases and end up padding their ontologies with extra knowledge in the context of algorithmic analysis.

Figure 1.1 depicts an objective map wherein we might divide a generic idealized KAM into five basic modules, or "agents": selection, organization, context map, display, and collaboration. Here, we also introduce the concept of a "JAN," or atom of knowledge, described by Wang et al. [40] as an "abstract object for all the general resources". The idea of a JAN is not entirely new, and, indeed, has existed under different names in the past; one might liken a JAN to a "knol" from Google's eponymous Knol project [2]. This project was intended to provide user-written scholarly articles, and "knols" were literally defined by Google as "a unit of knowledge." The term was also used to refer simply to an article within the Knol project. However, as of May, 2012, the Knol project has been discontinued [19] in favor of an upcoming project called Annotum.

The Selection Agent would be primarily concerned with how the user selects and views portions of the personal ontology, including JANs. This agent represents the "user interface" by which one directly interacts with the KAM system.

The Organization Agent would be concerned with intelligently organizing these JANs into 


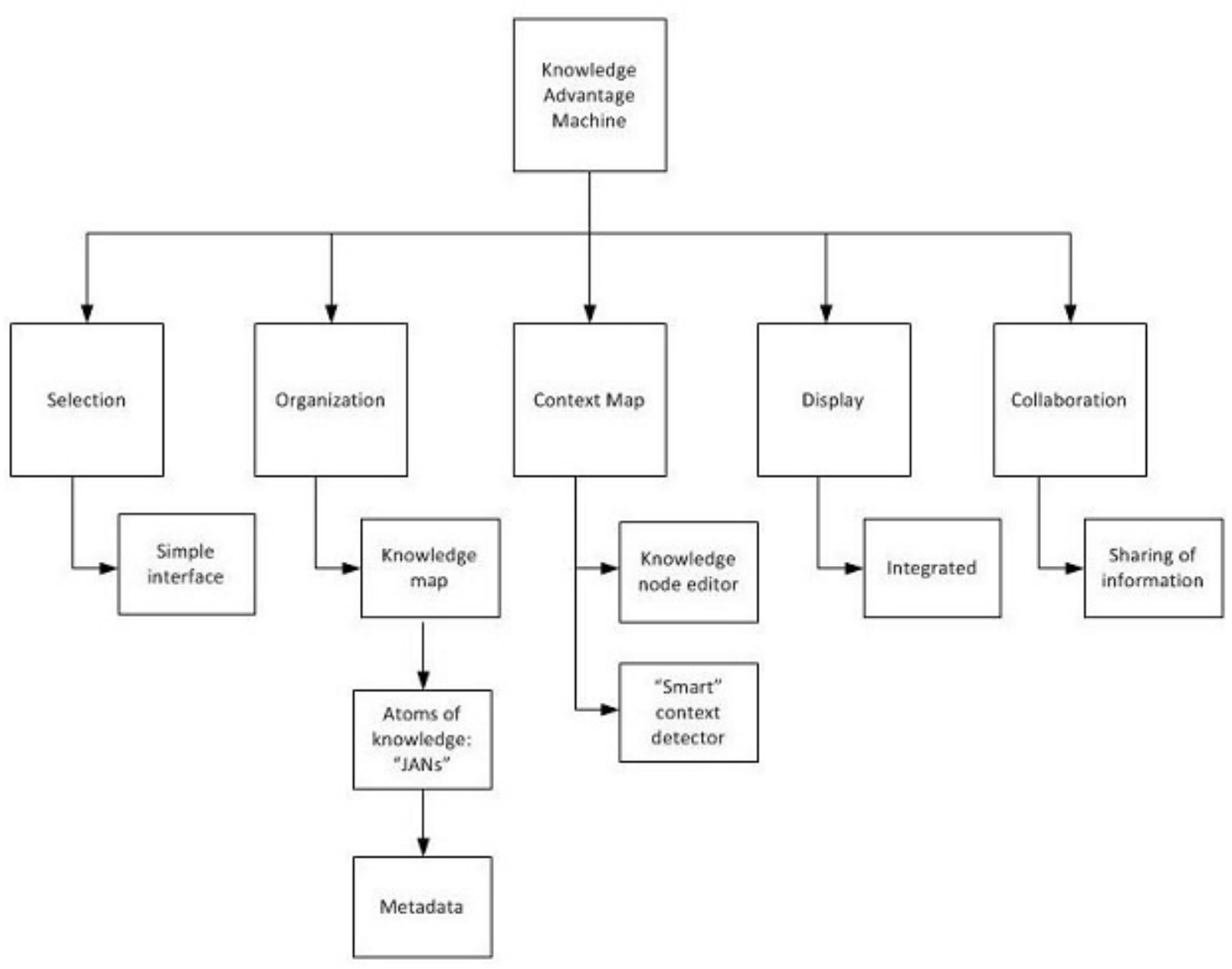

Figure 1.1: KAM Objective Map

semantically meaningful relationships within the personal ontology. This organization, in an idealized KAM, would be entirely (or mostly) automated, with manual organization being possible but generally unnecessary.

The Context Map Agent would be concerned with mapping information so as to assist in the intelligent identification of contexts. In an idealized KAM this would also be fully automated; for example, if a scientist has several research papers concerning nanotechnology within their personal ontology, the KAM should be able to automatically identify that these JANs are related and in the same context. The means of this identification vary; we might compare the semantic meaning of a 
set of documents, or we may (as in the focus of this thesis) examine file usage patterns, or another method entirely. An idealized KAM's Context Map Agent would also be capable of identifying which contexts a user is currently engaged in; for example, a researcher taking part in a meeting about nanotechnology would be identified as being in the "nanotechnology" context.

The Display Agent would be concerned with how the various aspects of the KAM are displayed to the user. This agent represents the other half of the user interface, and is primarily concerned with the method of displaying the user's personal ontology. One KAM prototype called "GKAM" [21] used a radial graph to implement the Display Agent.

Finally, the Collaboration Agent would be concerned with providing ways of sharing personal ontology information between users. This is perhaps the most important portion of a fully realized KAM, as "knowledge" must come from somewhere. A subsection of this agent, called the Discovery Agent, would be concerned with the automated discovery of new JANs which are associated with a user's personal ontology.

This is just one brief overview of how a KAM could operate; countless other "agents" could be envisioned for a functional KAM. Furthermore, each of these agents has complex implementation problems associated with it, and so for the purposes of this work we must focus on very specific aspects. This thesis therefore focuses on developing a portion of a functional Context Map Agent which, instead of examining the semantics of JANs in a user's personal ontology, attempts to identify a user's work-patterns by examining his or her file usage. This data can then be used to identify contexts and predict future file usage. The difference between these two approaches is simple, but crucial: both are attempting to gain a knowledge advantage, but the former concentrates on using domain information [40] to do so, and the second focuses on user-specific work-patterns. 


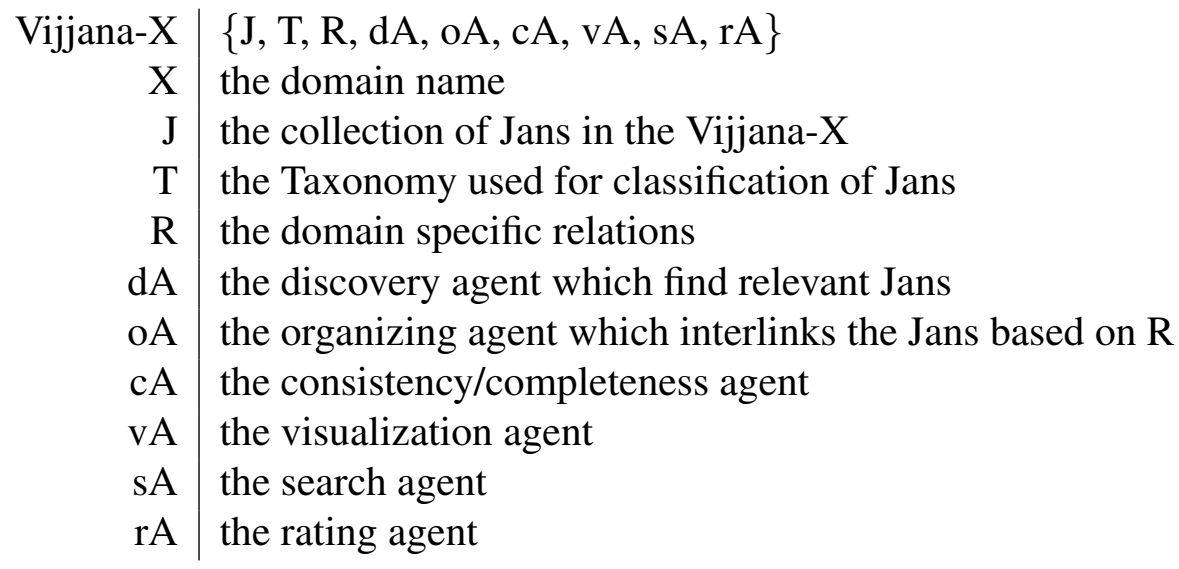

Figure 1.2: Vijjana Architecture [30]

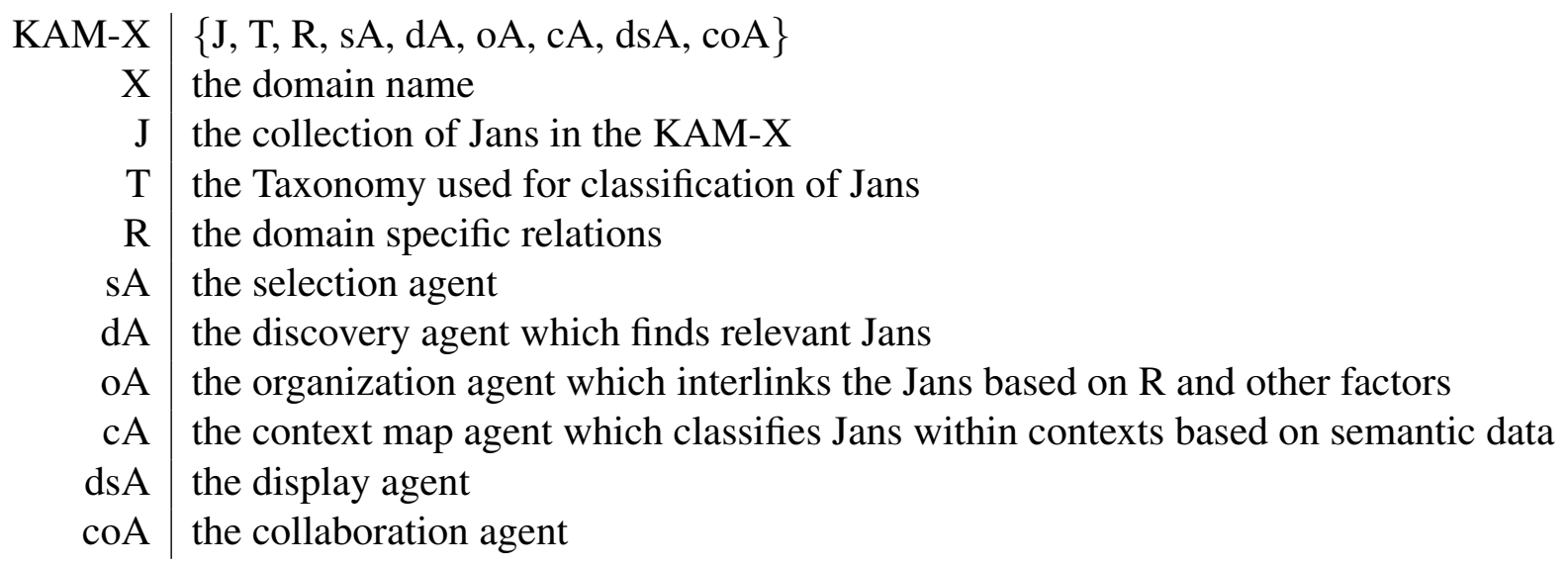

Figure 1.3: KAM Architecture 


\subsection{KAM Architectural View}

The architectural model for the KAM could be described in many ways. A good basis for this model is found within Vijjana [30]. Vijjana, presented by Reddy et. al, is a model which constructs a semantic-based knowledge network out of URLS2 Described in Figure 1.2, the Vijjana model introduces the concept of a JAN, and might be considered a predecessor to the KAM since it seeks to provide a knowledge advantage through a self-organizing knowledge network.

The architectural view of a generic idealized KAM model might similarly be described as seen in Figure 1.3 . This model presents a view of how the agents described in $\$ 1.2$ interact to facilitate the KAM. In $\$ 4.1$ this will be expanded to include work-pattern centric concepts.

\subsection{Contributions of Thesis}

The most salient contributions of this thesis follow:

- An overview of the KAM problem, its previous incarnations (such as the Context-Centric Knowledge Advantage Machine [40]), and its constituent parts

- An alternative model to the KAM's context predictor which instead functions through file access prediction

\subsection{Outline of Thesis}

The organization of the proceeding sections of this thesis is as follows:

- Chapter 2 offers the concrete objectives of this thesis. The method of inquiry and the actual research questions are described.

\footnotetext{
${ }^{2}$ Uniform Resource Locators
} 
- Chapter 3 examines the existing related literature. This includes literature concerning the semantic desktop, semantic web, web usage patterns, and ontological research. A description of a fully realized KAM is given. Finally, the main objectives of this research are revealed.

- Chapter 4 presents a detailed overview of the inner workings of the WPCKAM (Work Pattern Centric Knowledge Advantage Machine) file usage agent. This chapter describes how collected workflow patterns could be used to produce a knowledge advantage. Technologies used to create this agent are also described.

- Chapter 5 presents statistical data collected from running the passive monitoring agent on volunteers' computers for a period of one week. An analysis of the data is given, and an assessment of prediction metrics is performed.

- Chapter 6 details conclusions gathered from the analysis of the data. A commentary on the effectiveness of predicting work patterns is given. Afterwards, there is a discussion of the various avenues that could be pursued in future KAM-related research, as well as descriptions of several technologies which will likely prove useful for continued research.

- Appendix A explains how to replicate the experiments performed in this thesis. The means to acquire the actual program code and scripts are provided.

- Appendix $\mathrm{B}$ consists of various tables and charting of raw data. 


\section{Chapter 2}

\section{Research Objectives}

There are many avenues of research that could be pursued in regards to Knowledge Advantage Machines. This thesis focuses on work-patterns because they might act as an interesting alternative model to the KAM's context predictor [40] and they have yet to be utilized in that fashion. Thus, the purpose of this thesis is to create the basis for the WPCKAM: a type a KAM which is created with respect to work patterns. This thesis also aims to develop a roadmap towards scientifically examining this sort of system's impact on knowledge workers' efficiency in their everyday tasks.

The sections of this chapter are divided as follows:

- $\$ 2.1$ details the method of inquiry used to examine the efficacy of the WPCKAM learning and prediction tasks.

- $\$ 2.2$ explores the actual research questions that this thesis offers. Null and alternative hypotheses are offered which relate to these research questions.

\subsection{Method of Inquiry}

It is paramount to empirically demonstrate the results of our methods of examining and exploiting work patterns. We refer to this as the method of inquiry. Having collected a great deal of file usage 
data, we apply several different learning classifiers 1 to it. We first select the training set, which we define as the previous " $k$ " file accesses. The classifier is then trained on this set, and attempts to learn (i.e. predict) the next member of the set. In this way each file access "n" is trained on a set consisting of n-1, n-2, and so forth, up to n-k.

Next, the number of successful predictions versus failed predictions is tallied, and the process is repeated for various values of " $k$ ". Therefore, put simply, this research's core testing metric is how successful it is at predicting file usage. Our method of inquiry will therefore allow us to determine two important factors: which learning classifier is best at predicting file usage, and which value of "k" is ideal for predicting file usage.

Next, the number of successful filterings versus failed filterings is tallied. The top five predictions for a given classifier are examined, and if the correct prediction is within them, the prediction is considered a success. This secondary method of inquiry allows us to determine if the context can be "narrowed down" even if it cannot be predicted with good accuracy.

More specific information on the experimental design will be given in Chapter 5 .

\subsection{Research Questions}

The questions this research addresses follow:

- How accurate are file usage predictions based on work patterns using various classifiers?

- How accurate is filtering predictions for file usage based on work patterns using various classifiers?

As such, we identify our hypotheses as follows:

- Null Hypothesis 1: There is no difference in accuracy between predictions of file usage among various classifiers.

\footnotetext{
${ }^{1}$ Naive Bayes, J48, LWL, Hyperpipes, and OneR; see $\$ 3.6$
} 
- Alternative Hypothesis 1: There is a difference in accuracy between predictions of file usage among various classifiers.

- Null Hypothesis 2: There is no difference in accuracy between predictions of file usage among various $k$-values.

- Alternative Hypothesis 2: There is a difference in accuracy between predictions of file usage among various $\mathrm{k}$-values.

- Null Hypothesis 3: There is no difference in accuracy between filtering predictions of file usage among various classifiers.

- Alternative Hypothesis 3: There is a difference in accuracy between filtering predictions of file usage among various classifiers.

- Null Hypothesis 4: There is no difference in accuracy between filtering predictions of file usage among various $\mathrm{k}$-values.

- Alternative Hypothesis 4: There is a difference in accuracy between filtering predictions of file usage among various k-values.

\subsection{Summary}

This chapter has examined the objectives of the this thesis. The data mining techniques which serve as the method of inquiry were identified as the following classifiers:

- Naive Bayes

- J48

- LWL 
- Hyperpipes

- OneR

The means by which these classifiers are used to perform file prediction were explained. Finally, the research questions and their associated hypotheses were identified in this chapter. 


\section{Chapter 3}

\section{Background and Related Work}

The background of the "Knowledge Advantage Machine" concept resides mainly in the existing research behind the Semantic Web [4] and the Semantic Desktop [35]. The "jumping-off point" of KAM research is an attempt to create a middle-man between those two existing areas; we are concerned with semantically understanding both the web-content that knowledge workers might encounter, and the data-rich contents of their personal computers.

However, the main goal of this thesis is to be able to identify and exploit a given knowledge worker's "work patterns." We do this in order to facilitate one possible knowledge advantage machine. Other research has gone in different directions [40] [26]; this will be explored fully in the proceeding chapter.

In addition to examining existing Semantic Web and Semantic Desktop research, we must also establish a background for the "work-patterns" which we intend to study and harness for efficiency; in this way, a robust definition of a "work-pattern" can be defined. We will do this by making use of various data mining techniques; we will also attempt to gain insight into work pattern discovery by drawing comparisons to existing research into "web usage patterns" [38].

This chapter is therefore divided as follows:

- \$3.1 explains the background of the Semantic Web and how it relates to the Knowledge 
Advantage Machine.

- $\$ 3.2$ explains the background of the Semantic Desktop and how it relates to the Knowledge Advantage Machine. The "Semantic Desktop 2.0" is also described.

- $\$ 3.3$ offers a description of web usage mining [38] and how its constituent components are analogous to the sort of work pattern mining that the WPCKAM aims to achieve. Its subsections explore the three main steps of web usage mining: preprocessing, pattern discovery, and pattern analysis.

- $\$ 3.4$ offers exposition on Graph Based Induction, a learning model [26] which was used for various learning tasks including file prefetching.

- $\$ 3.5$ gives a background for ontological research, and how the concept of a personal ontology is core to the Knowledge Advantage Machine.

- \$3.6 explains what is meant by "classification learning" and how it pertains to this thesis. Each of the subsections explores an individual classifier used for this research.

\subsection{Semantic Web}

The Semantic Web, which introduces services which allow for machine-understanding of semantics on the Web, can be defined as something which "provides the infrastructure for the semantic interoperability of Web Services." Thus, with a Semantic Web in place, various Semantic Web Services can be developed to aid in automated discovery of information [4]. More recently, in 2008, the idea of using mobile devices in conjunction with the Semantic Web was explored [43]; as we have already shown, this is also a concept that is of interest in KAM research with respect to context aware computing. 


\subsection{Semantic Desktop}

An overview of the idea of the "Semantic Desktop" was published in 2005. This paper defined a semantic desktop as an attempt to "transfer the Semantic Web to desktop computers" - not just its actual technology, but the philosophy as well. The Semantic Desktop, therefore, attempts to accomplish the same goals as the Semantic Web via a desktop computer; specifically, the purpose is to give machines a semantic understanding of the contents of ones computer or files [35].

In 2006, the Semantic Desktop 2.0 was discussed, as was the idea of using metadata to organize data resources. This proposed "second edition" of the Semantic Desktop attempted to improve on the original design by using PIMO, the Personal Information model, as an approach for ontology organization. This model made it possible for tagging services to be created on the desktop. [34]

\subsection{Web Usage Mining}

In 1995, Letizia was implemented as an agent which worked with web browsers, specifically Netscape. It attempted to remember a user's patterns with concern to web browsing, taking note of things that user is interested in and using this information to recommend certain online documents that pertain to previous searches [22].

A great deal of research has been done in the area of applying data mining techniques in order to establish "web-usage patterns" - this is sometimes referred to as "web-usage mining" [38]. Accordingly, within this section I will explore the feasibility of using a similar plan in order to perform what we will refer to as "work-pattern mining."

In an idealized KAM, work patterns could be discovered in a manner based on the web usage mining techniques used by Srivastava et al [38]. In their paper, usage patterns are shown to be "mined" in three phases: preprocessing, pattern discovery, and pattern analysis. 


\subsubsection{Preprocessing}

Preprocessing is described [38] as consisting of taking content, structure, and usage information and abstracting them into metadata which allows for pattern discovery. Therefore, preprocessing happens in three stages, which I will first describe, then explore how these concepts might be used as analogues that are viable in the WPCKAM framework.

In web usage mining, usage preprocessing generally consists of identifying the user and dividing the click-stream into sessions. In an idealized KAM framework, the user might not need to be identified, but information analogous to a "click-stream" could be useful. In a WPCKAM system, the analogue to a clickstream could be described as the timestamps, filenames, and other metadata associated with the files a user is accessing and editing.

Content preprocessing consists of converting the actual content (text, images, multimedia, etc) into other forms which are more useful for web usage mining. Likewise, the various file content which an idealized KAM user interacts with on a regular basis must be converted into forms which are useful for work pattern mining. This content might not just consist of the actual contents of the files which have been altered, but also the differences between the newly modified file and the original. Specifically, [16] made use of vector space model to place the content in a quantifiable format, and one might likewise use a semantic space and Latent Semantic Analysis [7] to examine the actual content of files.

Structure preprocessing consists of examining the structure (i.e. the "hypertext links between page views") of a site, then preprocessing it in a similar manner as content preprocessing above. Since the WPCKAM framework is primarily concerned with filesystem access and content rather than web content, there is no real analogue. Thus, this preprocessing step is omitted in our system. 


\subsubsection{Pattern Discovery}

The second step of web usage mining, "pattern discovery," is described as drawing upon methodologies from many fields, including pattern recognition, data mining, and statistics [38]. We will likewise draw from the same base of methods (especially data mining) in order to perform our "work-pattern mining." This step consists of several sub-steps: statistical analysis, association rule generation, clustering, classification, sequential pattern discovery, and dependency modeling.

In the WPCKAM framework, statistical analysis of the work patterns is performed via classification learning techniques. [38] refers to basic statistical techniques used to find the mean, median, etc for values such as the most frequently accessed page views, viewing time, etc. Likewise, when searching for work patterns we might also record most frequently accessed files, access times, and so forth.

Association rule generation is intended to relate pages which are referenced together in one server session. The analogue here is that we will instead relate files which are accessed together in one "session" or "K-set' "1] Specifically, [38] attempts to identify sets of pages which are commonly accessed together at rates above some specified value. Thus, using statistical analysis or data mining techniques we might also specify some threshold which will allow us to determine whether we can consider two files to be typically accessed "together" by a particular user.

Clustering is an establish data mining technique which simply groups together items which have similar characteristics. For web usage, [38] was primarily concerned with establishing two clusters: usage clusters and page clusters. Usage clusters were clusters of users which apparently had similar browsing habits, and page clusters are clusters of pages with related content. Both clusters are directly analogous to our KAM framework; in an idealized KAM we might find clusters of users with similar work patterns, and clusters of files with related content. However, for the WPCKAM, we are already performing a form of clustering by virtue of using the Locally Weighted

\footnotetext{
${ }^{1}$ The previous "K" file accesses which act as the current training set for the learning classifier.
} 
Learning $2^{2}$ classifier which makes use of the k-means clusterer.

Classification learning is another data mining technique which will be detailed extensively in $\$ 3.6$. For web usage, [38] sought to classify users. In the WPCKAM framework we instead attempt to perform classification on files in order to predict future file usage. An idealized KAM might also classify users into various categories.

Sequential pattern discovery is defined as an attempt to find "inter-session" patterns. In web usage mining, this might consist of some specific set of items which is proceeded by some other specific item within an overall set of sessions. Likewise, we might find sequential patterns in workpattern mining; this is essentially the core concept insofar as we are attempting to predict which files within the user's personal ontology are most likely to be accessed in the near future.

Dependency modeling is the sixth and final step of pattern discovery. This consists of attempting to build models which are "capable of representing significant dependencies among the various variables in the Web domain. [38]" An example given is the stages a potential customer might go through whilst shopping online; these might be used to identify a casual visitor or an actual buyer. Since this is primarily concerned with navigation, there may be no good analogue in the WPCKAM framework. On the other hand, in an idealized KAM (with full knowledge of the user's activities) we might liken this to the actual steps a user takes while working; for example, the user first searches and researches a subject, then a document is accessed, then the same document is edited.

\subsubsection{Pattern Analysis}

The third and final step of web usage mining, "pattern analysis," focuses on the filtering of patterns or rules which are "uninteresting." Anything that was found within the pattern discovery phase is examined and discarded if it is deemed to be useless. This is our exact goal in work-pattern

\footnotetext{
${ }^{2}$ See $\$ 5.4 .4$
} 
mining. [38] describes various methods of accomplishing this, such as the use of data cubes 3 , which allow for On-Line Analytical Processing (OLAP) operations.

OLAP is known to be an effective method of analyzing very large amounts of data [27]. However, [38] ultimately asserts that the analysis method should be governed by the application one is performing web mining on. Thus, in our analysis of work-patterns, we will design our own methodology wherein analysis is performed through data mining classification techniques.

\subsection{Graph Based Induction}

In 1998 Graph Based Induction [26], or GBI, was proposed as a learning model in order to perform various learning tasks, including file prefetching via a user-obscured "Prefetch daemon" tool. File prefetching is a potent technique which works by predicting soon-to-be-used disk blocks so that they can be "prefetched" into memory, increasing efficiency. Shriver et al. [36] showed that application throughput can be improved by up to fifty percent via file prefetching.

GBI is able to "extract regularities" from a graph of user-dependent data using top-down induction. It was originally designed to examine inference patterns and extract any patterns which appear frequently within the inference trace. GBI is, therefore, looking for "regularities" in input traces, allowing the algorithm to hasten tasks such as learning and classification.

The algorithm works finding patterns, then contracting the graph after replacing the found pattern with single new node. However, the graph cannot ever contract so far as to become a single node, because the graph size is defined by both the sizes of extract patterns and the size of the contracted graph. This core "contraction" technique used in GBI is called pairwise chunking, and is shown in Figure 3.1. Pairwise chunking works within the overall GBI algorithm to "chunk" appropriated linked pairs of nodes at each step of any search.

GBI's "extracted regularities" come in the form of subpatterns. The algorithm only returns sub-

\footnotetext{
${ }^{3}$ Sometimes known as OLAP cubes.
} 

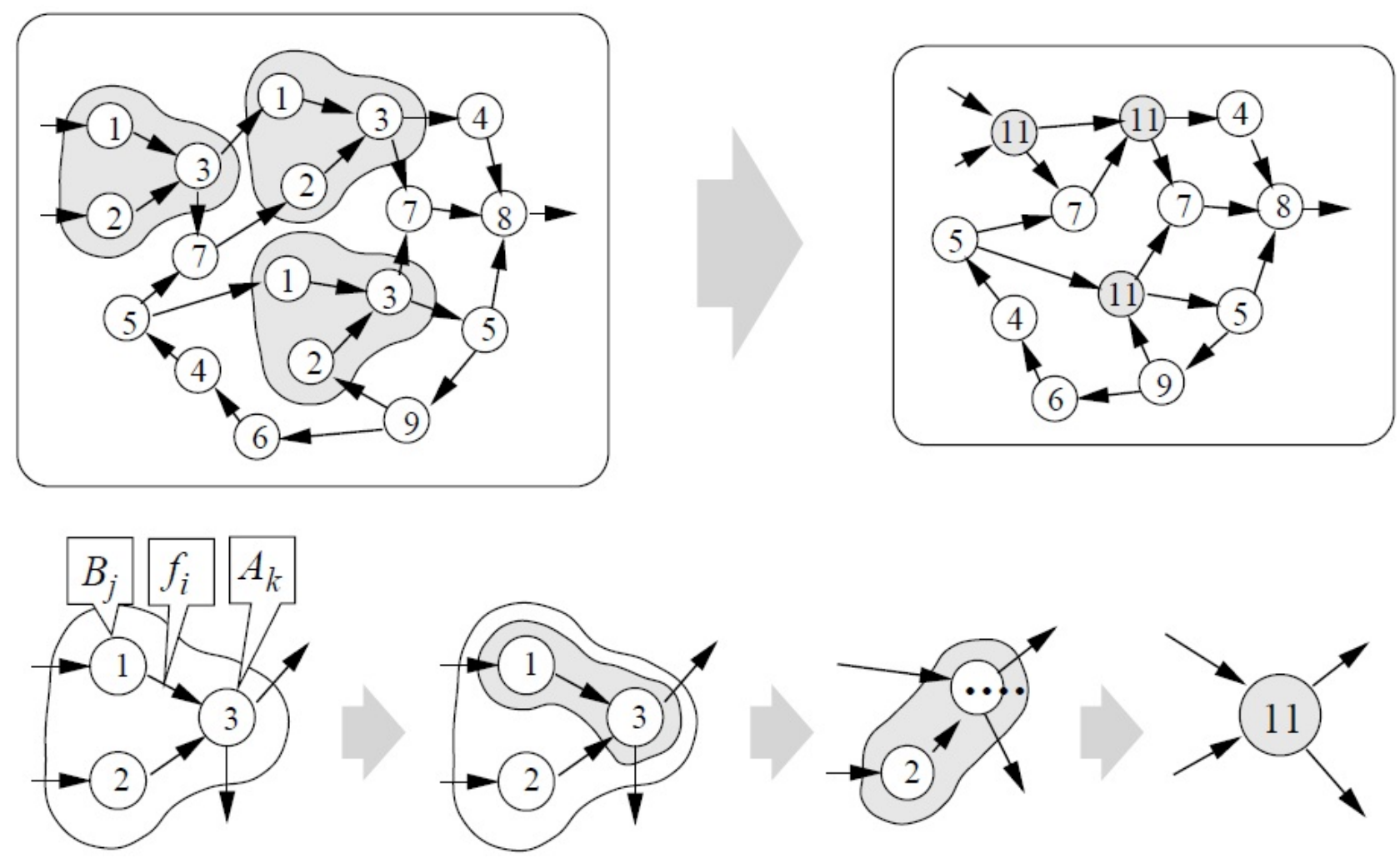

Figure 3.1: Pairwise Chunking [26]

patterns where the graph size was ultimately minimum; these are considered to be the "interesting" patterns.

\subsection{Ontological Research}

Ontology is defined as the study of the nature of existence. A "formal ontology" can be described as a designed structure or graph whose purpose is supporting knowledge sharing activities; this concept could be used in such knowledge-intensive work as engineering and mathematics [13].

Chen et al. [6] described "COBRA-ONT4," a type of ontology specifically designed to support systems which are context-aware. [6] describes the movement of computing towards these context-aware systems and the need for an ontology that supports them. "Intelligent agents" are

\footnotetext{
${ }^{4}$ Context Broker Architecture Ontology
} 
described, such as the ones described in $\$ 1.2$, which are capable of intelligently understanding context information and using this information to support various applications.

When referring to KAM research, it is important to be familiar with the concept of a "personal ontology," which is an overall description of an individual via his or her interests. This thesis uses the phrase "knowledge base" interchangeably with "personal ontology"; put as simply as possible, we view a personal ontology as the sum total of an individual's collected knowledge, which can be discretized into clusters called contexts and tagged with metadata to describe the semantic contents.

\subsection{Classifiers}

Several classifiers were tested in order to perform learning tasks for this research. Statistical classification is defined as the process in which a training set of data is examined, and a new observation is placed into one of a set of several existing "categories." Likewise, classification learning in data mining examines a set of classified examples (generally still referred to as the training set), with the intent of learning how to classify unknown examples. Throughout this thesis we will refer to the " $\mathrm{K}$ " value, which is the number of previous examples in the training set that will be examined to prepare for classification. The experimental design significance surrounding this "K" value will be described in Chapter 5 .

Classification learning in data mining is considered a kind of "supervised" learning [42]; this simply means that, unlike unsupervised learning methods such as clustering, classification training sets always provide the true outcome (or "class") of each training example.

In this research, we are concerned with predicting file usage, and so our categories are defined as the files which are being accessed, and the new observation is/are the file(s) which have yet to be accessed. While "multilabeled instances" do exist for some scenarios - that is, classification examples which belong to multiple classes - for the purposes of this thesis every example must 
exist in only one class because each item may only have one file location.

Classification algorithms are ubiquitous in data mining research. As shown by Giudici [12], classification learning and other data mining techniques have been applied real world scenarios, such as business and industry problems. The "Bayes' Theorem" that Naive Bayes classification employs has been used in myriad fields [25], including codebreaking, DNA de-coding, and Homeland Security tasks. Bayesian approaches have even been used in order to filter through junk mail [33].

The efficiency of these classifiers in extracting relevant file usage patterns will be examined in Chapter 5, here, I provide a brief overview of how each of the studied classifiers functions. The following classifiers were not implemented directly; instead, implementations from WEKA [14:5 were used.

\subsubsection{Naive Bayes}

Naive Bayes is a type of probabilistic classifier which works around Bayes's theorem. Bayes' theorem, also known as "Bayes' rule" [17], specifies a relationship between the probability of some conditional hypothesis on some set of data with the inverse probability of some conditional data on the hypothesis. The theorem can be expressed in several ways, such as with the symmetry principle for probability ratios seen in Figure 3.3

Naive Bayes itself is a simple and efficient [41] classifier which, despite its power, operates on an ostensibly "naive" principle: observed events occur entirely independently of each other. This assumption is considered naive because it is generally false. The Naive Bayes classifier calculates probabilities of examples being in a particular class [44]; the classifier can ultimately be depicted as seen in Figure 3.4 .

Naive Bayes which has been empirically shown by Rish [31] to typically be extremely effective in comparison to other more advanced classifiers, despite its generally flawed assumption of in-

\footnotetext{
${ }^{5}$ Described in $\$ 4.3 .2$.
} 


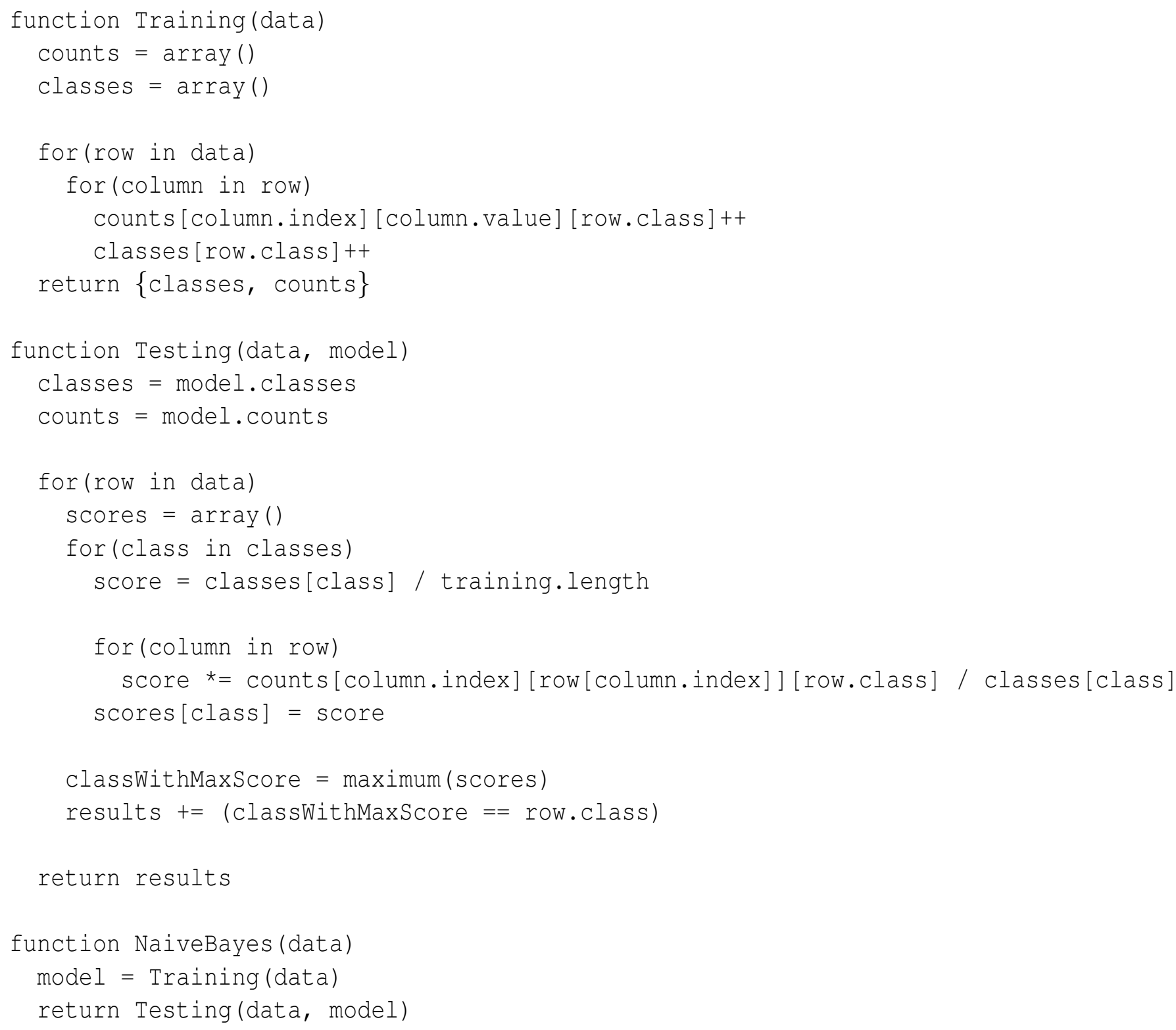

Figure 3.2: Pseudo code for Naive Bayes [20]

$P R(H, E)=P R(E, H)$

Figure 3.3: Symmetry Principle for Probability Ratios [17] 


$$
f_{n b}(E)=\frac{p(C=+)}{p(C=-)} \prod_{i=1}^{n} \frac{p\left(x_{i} \mid C=+\right)}{p\left(x_{i} \mid C=-\right)}
$$

Figure 3.4: Naive Bayes Classifier [44]

dependence. This empirical study showed that, in spite of inaccurate probability estimates, Naive Bayes's classification decisions were often correct.

It was also demonstrated that the best performance of Naive Bayes occurs when features are completely independent (i.e. the "naive" assumption of independence is actually true) and, interestingly, when features are functionally dependent. When features are only partially dependent, Naive Bayes has poorer performance. Therefore, we might expect Naive Bayes to perform well for file usage prediction if each atom of file usage is totally unrelated to the others, or if they are totally dependent on each other.

\subsubsection{J48}

J48 is WEKA's open source Java implementation [14] of Ross Quinlan's decision tree generating algorithm called C4.5 [29]. As with most classifiers, a training set of previously classified samples is provided to the algorithm. C4.5 then builds decision trees based on this training data. One attribute of data is chosen for each node of the tree, based on which attribute best separates the sample set into subsets of classes. Information gain is measured for the purposes of this separation; that is, the attribute that has the highest information gain is used to create the separation, and the algorithm proceeds recursively until it reaches one of three base cases, shown in Figure 3.5 .

\subsubsection{LWL}

LWL stands for Locally Weighted Learning, and functions as a locally weighted version of Naive Bayes [10]. This local weighting serves to "relax" the typically naive assumption of attribute 


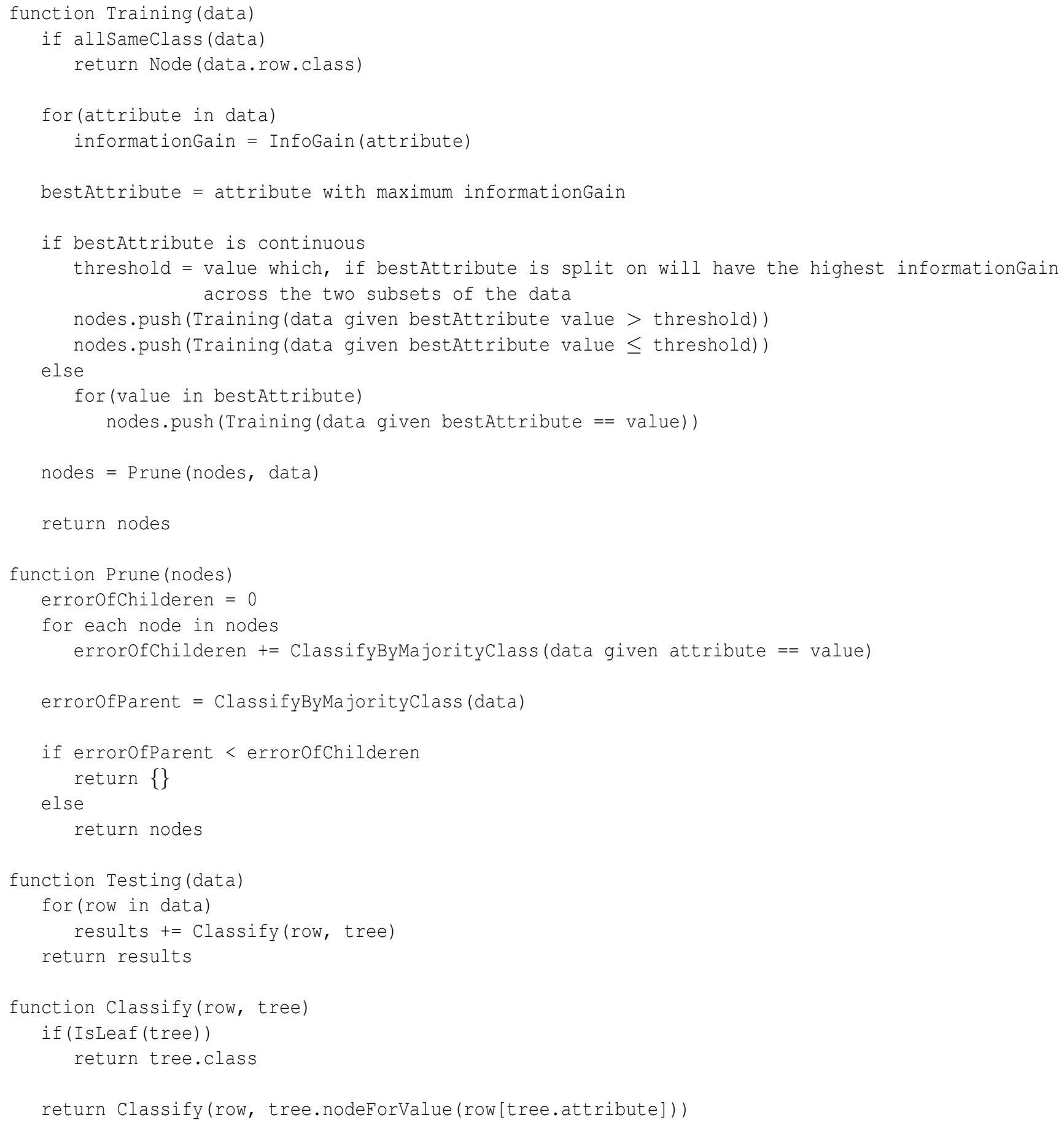

Figure 3.5: Pseudo code for C4.5 [20] 


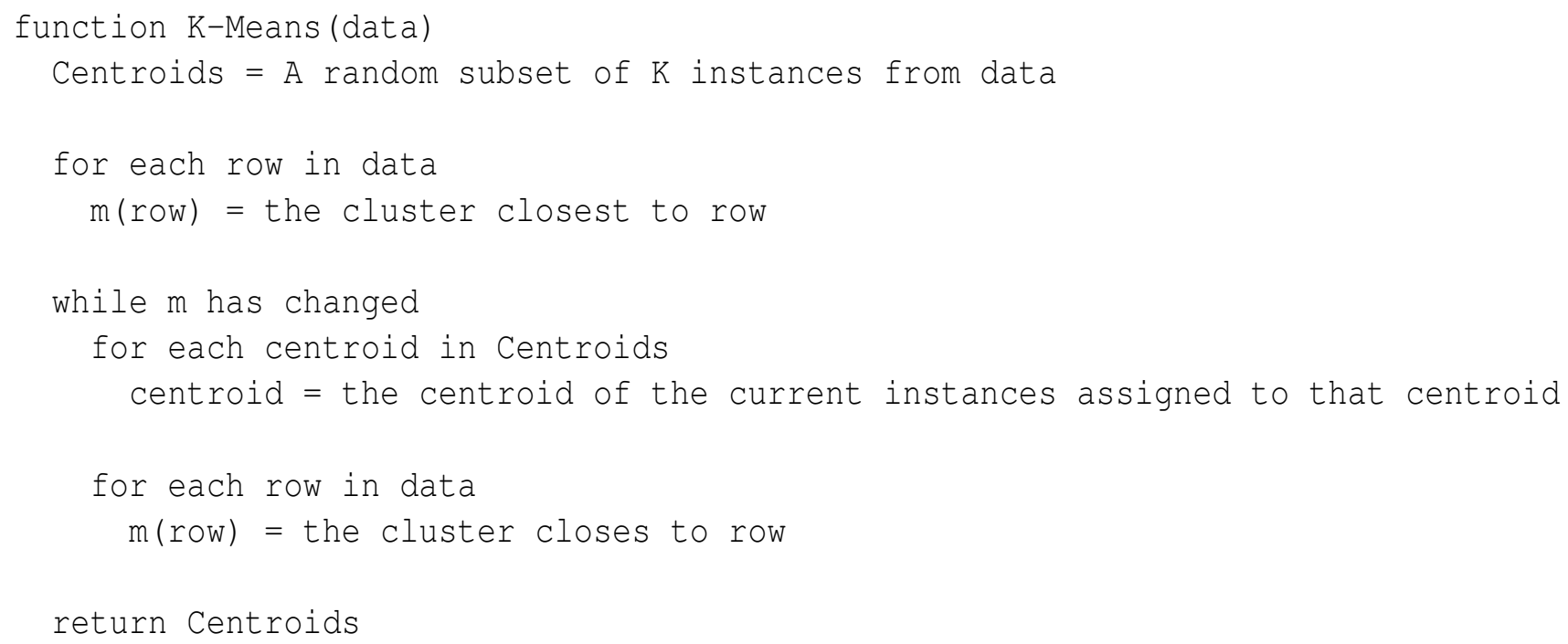

Figure 3.6: Pseudo code for K-Means [18]

independence, as local models are learned at prediction time. This is sometimes called a "lazy" learning approach because of how the learning effort is deferred. LWL's performance is typically not impacted by the $\mathrm{k}^{6}$ value of the inherent $\mathrm{k}$-means clustering algorithm used to perform the local weighting. The "k" in k-means clustering represents the number of clusters that the observations will be divided into. [24].

\subsubsection{Hyperpipes}

Hyperpipes is a learning classifier created by Lucio de Souza Coelho and Len Trigg [42]. This is a rule-based learner, and is based on having only one simple rule per class. For each category attribute in the training data, a range of values is observed. The "rule" is then to decide which ranges have the attribute values of a particular test instance; the category that has the most correct ranges is then chosen.

Because of its simplicity, Hyperpipes performs classification quickly when there are large numbers of attributes [8]. It was shown in [8] that Hyperpipes outperformed several other classifiers.

\footnotetext{
${ }^{6}$ Not to be confused with the K value described at the beginning of this section.
} 


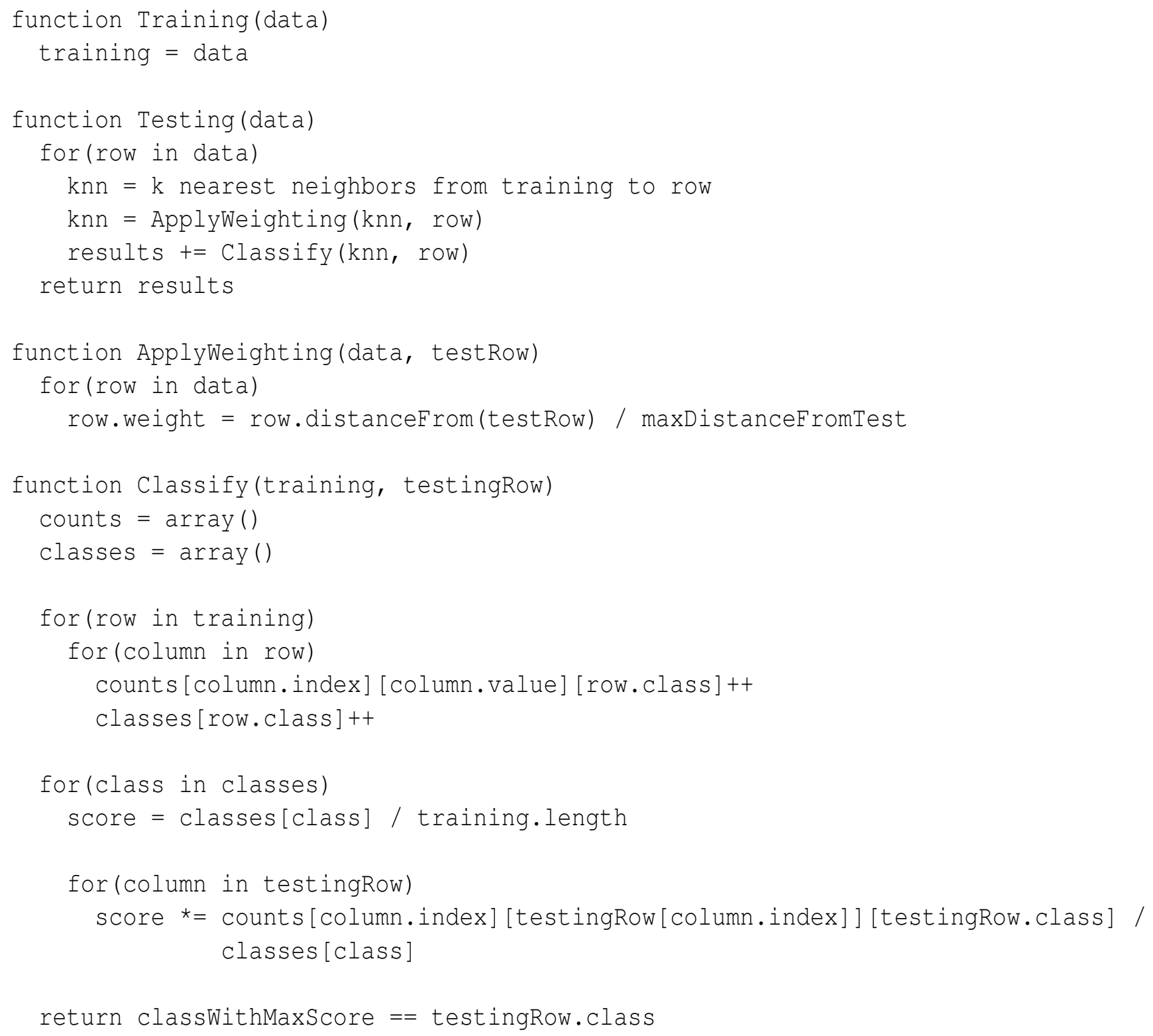

Figure 3.7: Pseudo code for Locally Weighted Naive Bayes [20] 


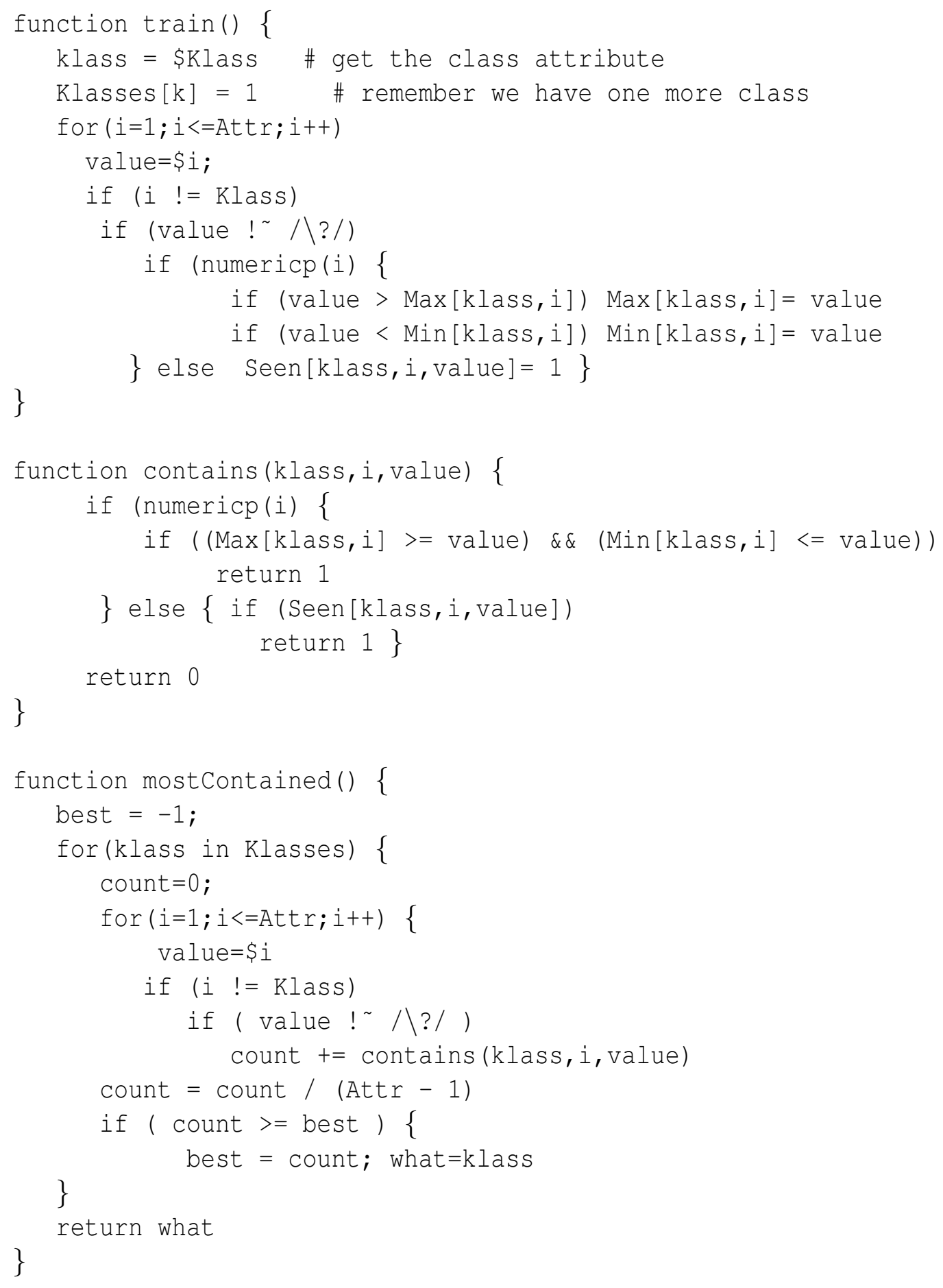

Figure 3.8: Pseudo code for Hyperpipes [11] 


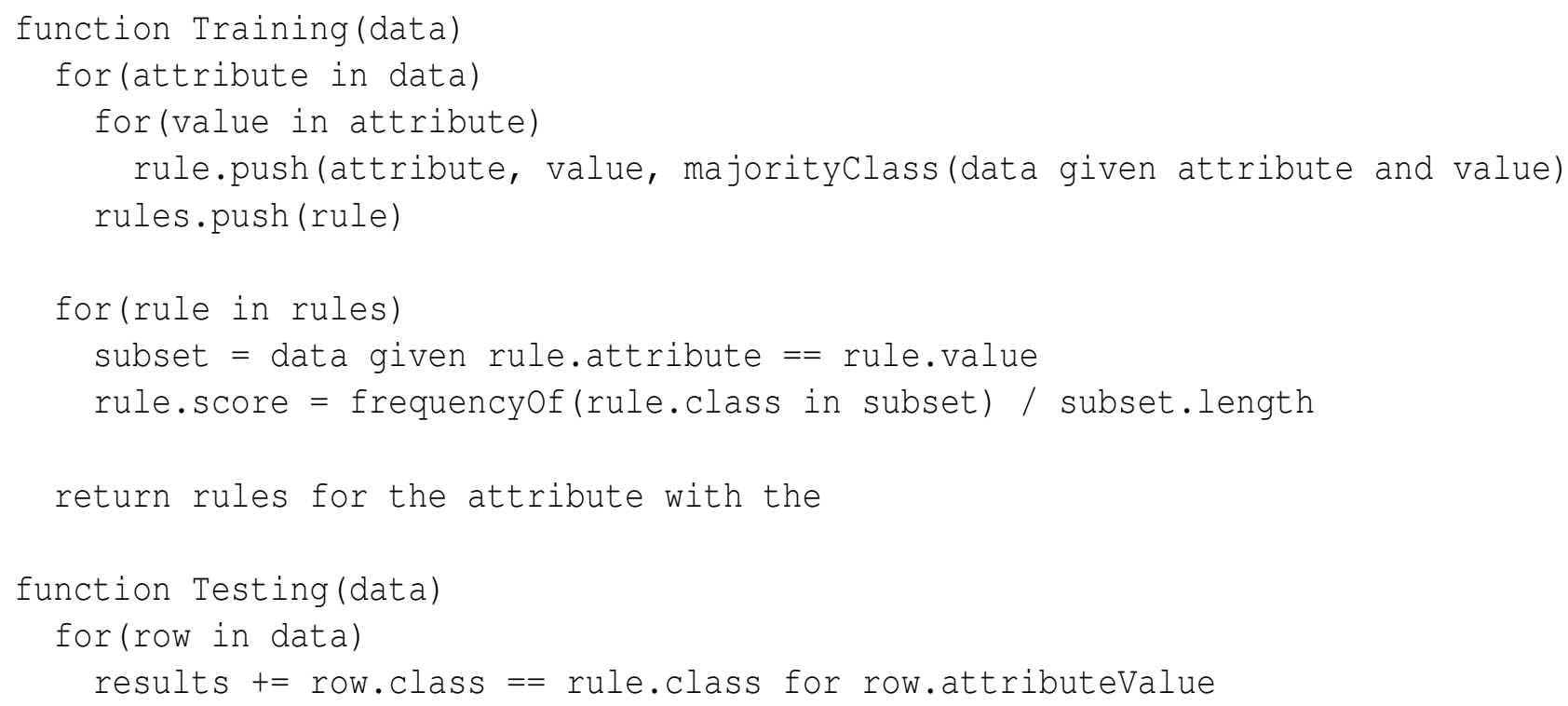

Figure 3.9: Pseudo code of OneR [20]

\subsubsection{OneR}

OneR, or "One Rule" classification has been shown to work well for most commonly used datasets [15]. This classifier works by creating only one rule for each attribute. This is a rule based learner which states that the majority class is $\mathrm{C}$ for any attribute $\mathrm{A}$ and value $\mathrm{V}$. After training, the accuracies associated with the created rules are then examined with respect to hypothesis $\mathrm{H}$. Accuracies below the majority class $\mathrm{C}$ are eliminated.

The algorithm is such that a singular poor choice of rules can drastically reduce classification accuracy. This learner is therefore not expected to perform well; it is included for comparison purposes only. Pseudo code for OneR can be found in Figure 3.9 .

\subsection{Summary}

This chapter has reviewed the literature pertaining to the Knowledge Advantage Machine and the building blocks that have made it possible. The Semantic Web and Semantic Desktop were 
reviewed, as well as the background of ontological research. Web usage mining was examined piece-by-piece and its constituent steps were compared to the KAM framework.

Various learning techniques were explored, including:

- Graph Based Induction

- Naive Bayes

- J48

- LWL

- Hyperpipes

- OneR 


\section{Chapter 4}

\section{Work Pattern Centric Knowledge}

\section{Advantage Machine}

In this chapter, we describe the WPCKAM framework, its goals, and its associated technologies. Thoughts on how the WPCKAM's contributions could be used to help implement an idealized KAM are also offered.

This chapter is therefore divided as follows:

- $\$ 4.1$ presents the architectural model for the WPCKAM.

- $\$ 4.2$ offers a brief description of file usage prediction techniques.

- $\$ 4.3$ and its subsections describe the technologies used to create and analyze various aspects of a prototype WPCKAM, including WEKA [14], the data mining suite.

- $\$ 4.4$ shows examples of how a knowledge advantage can be more easily gained through work-pattern centric elements. This section demonstrates how WPCKAM concepts relate back to the greater problem of creating an idealized KAM. 


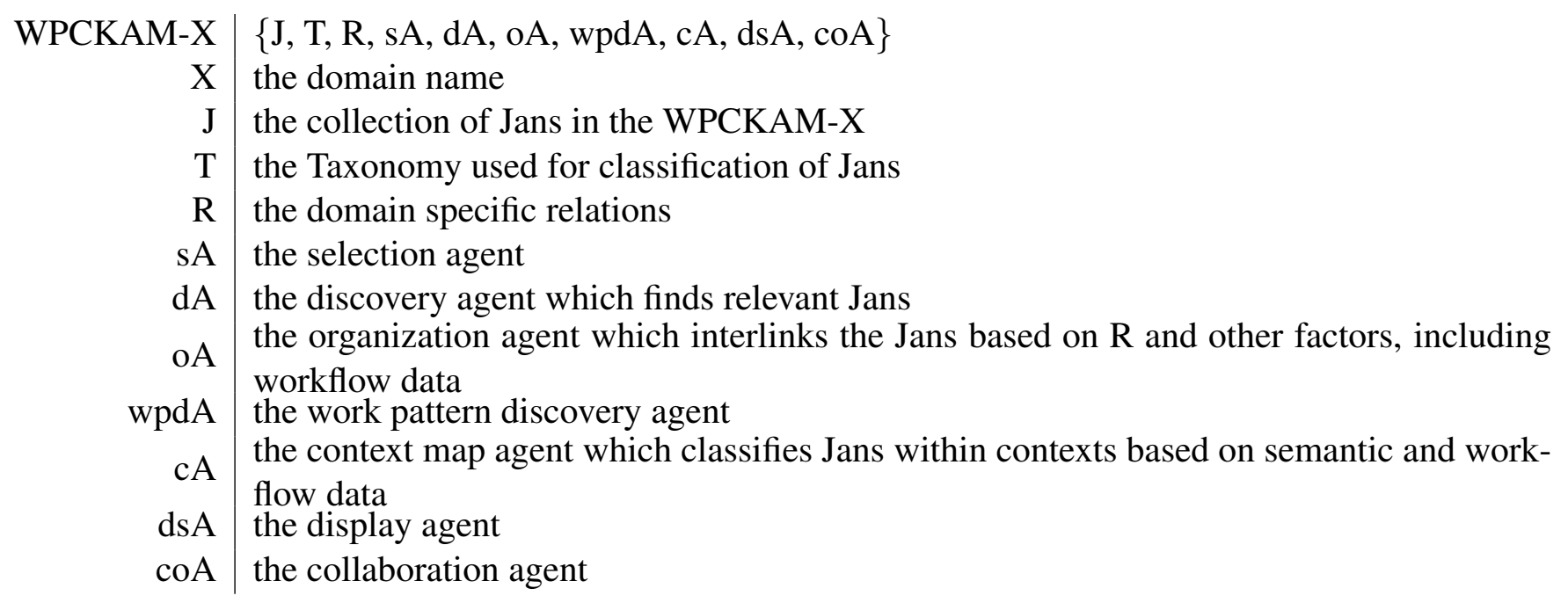

Figure 4.1: WPCKAM Architecture

\subsection{WPCKAM Architectural View}

The architectural view for the WPCKAM necessarily differs from the KAM's model, as we must now account for file prediction. This model therefore demonstrates how workflow pattern prediction could be used within a KAM to produce a knowledge advantage. We see in Figure 4.1 that the model is similar to the one presented in $\S 1.3$, with the utilization of workflow knowledge added.

A new agent, the "Work Pattern Discovery Agent," is introduced; this module would be responsible for passively monitoring the filesystem (or the user's personal ontology) as described throughout this thesis, and storing statistical data or training sets pertaining to the collected data. The context and organization agents would now be responsible for properly husbanding this data in various ways.

\subsection{File Usage Prediction}

In this thesis, "file usage prediction" is centered around the idea that knowing what has been used before can predict what will be needed. As a simple example, we might examine the case of a person getting ready for work: on a daily basis, they wake up, shower, eat breakfast, and brush 
their teeth in that order. While this pattern might exhibit some fluctuation, such as if a person is running late and skips breakfast, the overall pattern will remain the same. We could therefore expect similar patterns to arise from workflow data.

In this thesis, file usage prediction is meant to primarily act as an alternative context predictor. It could therefore be described as an alternative context map agent ${ }^{1}$ Because file usage prediction is one of the main goals of this research, it is imperative to examine appropriate methods of implementing it.

A decision making process such as $\mathrm{MDP}^{2}$ [28] would, at first, seem to be the ideal solution for these sorts of predictions. However, our system fails to obey the "Markov Property" - that is, a property that demands our system be "memoryless." This stochastic process makes decisions based solely on the current state and on how far we intend to look ahead; for the WPCKAM, our goal is to instead base the decision process on past file usage choices.

GBI [26] has also been used, as described in $\$ 3.4$, but was interwoven with interface and command data, and was designed for alternate purposes.

We therefore instead choose to use a variety of classifiers, described in $\$ 3.6$, to perform file usage prediction via classification learning. In Chapter 5 the complete experimental setup of these classifiers is described, as well as an analysis of the resulting data.

The motivation for using a variety of classifiers is simple: each represents a different approach to learning from the data. We survey various classifiers in order to discover which will be "best" for workflow prediction and the WPCKAM overall. As described in $\$ 3.6$, classifiers tend to work differently on different types of sets. Furthermore, some classifiers are known for their speed, such as Hyperpipes [14], and others are shown to be superior for their general prediction accuracy [31]. It is therefore important to take a variety of criteria into account, and using multiple classifiers allows a more robust survey of prediction metrics.

\footnotetext{
${ }^{1}$ See $\$ 1.2$.

${ }^{2}$ Markov Decision Process
} 


\subsection{Technologies}

The proceeding subsections will explore various pertinent technologies that were used to assist in workflow prediction activities. These technologies could also prove useful in an idealized WPCKAM system.

\subsubsection{Windows Auditing}

Various versions of Windows contain an auditing system [37]. This system can be used for a variety of purposes to track various activites on a computer. For this research, file auditing was used to keep track of file usage data, therefore monitoring workflow. This workflow data was saved in XML format. Finally, before analysis, a variety of preprocessing steps are applied to the XML; this process will be described in depth in $\$ 5.2$. The end result of these preprocessing steps, however, is a simple list of file accesses.

\subsubsection{WEKA}

WEKA [14] is a Java-based open source software package designed to provide various data mining utilities. This package offers implementations of various classifiers, clusterers, assocation learning utilities, and other learning tools. It also contains implementations of various experimentation and statistical tools such as paired t-tests. For this thesis, WEKA's implementations of the classifiers J48, Naive Bayes, Hyperpipes, and LWL were used to perform classification learning on the file usage data sets in order to learn work patterns.

\subsection{How the Advantage is Gained}

The core concept of the KAM is producing the aforementioned "knowledge advantage." Therefore, when describing a WPCKAM, we are primarily concerned with identifying how the "work-pattern 
centric" elements are producing that advantage. In a fully realized KAM, work-patterns discovered within the WPCKAM framework could be utilized in several ways. This section will examine several areas where this strategy could be employed to produce a knowledge advantage.

The subsections are therefore divided as follows:

- $\$ 4.4 .1$ shows how workflow prediction could be utilized.

- $\$ 4.4 .2$ examines the possibility of presenting predictions directly to the user as an alternative route to file access.

- $\$ 4.4 .3$ discusses how file usage data can be used to establish a metric for the importance of JANs within a personal ontology.

\subsubsection{Context Awareness}

As demonstrated in the sample scenario in $\$ 1.1 .1$, context awareness is integral for a KAM to produce a knowledge advantage. File usage prediction offers an interesting alternative to a typical KAM's context prediction. Typically a KAM identifies similarity between JANs by semantically analyzing a user's entire knowledge-base and comparing the content in order to establish a metric of comparison; for example, one project called GKAM [21 $]^{3}$ used TFIDF ${ }^{4}$ calculations to accomplish this.

Instead, the WPCKAM could identify a JAN's "contextually similar" JANs as those that are most frequently accessed together. Alternately, these contextually similar JANs could be identified as the "N" JANs that are most likely to be predicted after a particular access.

It would also be possible to use the probablility as a modifier for relatedness detection within the context map or organization agent. Access probability could be coupled with a semantic-centric

\footnotetext{
${ }^{3}$ Graphical Knowledge Advantage Machine

${ }^{4}$ Term Frequency Times Inverse Document Frequency
} 
metric (such as TFIDF as in GKAM [21]) in order to weight probable related documents without eliminating related but under-accessed documents.

These alternative methods for identification of context could prove more effective than context awareness accomplished solely through semantic analysis, and therefore bear further investigation. This thesis establishes a basis for that investigation by providing empirical results from file prediction via classification.

\subsubsection{Presenting Predicted Files}

With an agent capable of predicting file accesses with a high degree of success, some new methods of producing a knowledge advantage become possible for the KAM. One simple way to harness file prediction would be providing the WPCKAM's user with a small application which continuously shows likely-to-be-accessed files within a panel. This potential application offers a centralized location where files can be accessed more conveniently and quickly, providing a small but relevant advantage.

An alternative, less intrusive method of presenting these predictions would be to populate a folder with links to files which are predicted to be accessed in the near future.

\subsubsection{Measuring JAN Importance}

With access to file usage data, we might proceed with the reasonable assumption that the most frequently accessed JANs are more "important" to the user. A KAM with access to this data could therefore automatically know the elements of a user's personal ontology which are most important to them. These "important" JANs could be tagged as such, and semantic analysis agents could later be employed to glean the semantic significance of those particular JANs. Some form of discovery agent ${ }^{5}$ could then be used to discover related JANs from outside the personal ontology, offering

\footnotetext{
${ }^{5}$ See $\$ 1.2$.
} 
them to the user and potentially producing a knowledge advantage.

\subsection{Summary}

This chapter has described the Work Pattern Centric Knowledge Advantage Machine and its architectural model. The motivations behind the choice of file usage prediction metrics were discussed. Technologies used to create this thesis's experimental contribution were also examined. Finally, this chapter explored how work-pattern centric elements could be used to leverage a knowledge advantage within a fully realized KAM.

In the following chapters, the discussion will move to research objectives, the experimental setup, and the findings for the various classifiers when used for the purposes that have been described in the previous chapters. 


\section{Chapter 5}

\section{Experimental Setup and Analysis of Results}

This chapter offers an overview of the experimental methods, some raw results across data sets, and how these results were analyzed.

This chapter is therefore divided as follows:

- $\$ 5.1$ gives a brief overview of the experimental setup,

- $\$ 5.2$ displays an in-depth view of the preprocessing steps that the collected data was subjected to before classification.

- $\$ 5.3$ demonstrates the actual results of the data collection. Several tables of data are provided, as well as box plot charting

- $\$ 5.4$ offers thoughts on the performance of the individual classifiers based on the data across all sets. This section also gives some implementation-specific details for these classifiers.

- $\$ 5.5$ consists of more in-depth statistical analysis performed on the data. 


\subsection{Overview of Setup}

The experimental setup for this research consists of three main parts: data collection and preprocessing, classification/prediction, and data analysis. Data collection is accomplished using Windows object access auditing [37]. The means for this have been described in $\$ 4.3$. The data was collected from a group of volunteers gathered from various science and information fields. These volunteers were all running Windows 7 Professional.

In order to attempt to find interesting results, various values of "k" (\$5.2) are examined in various increments from $\mathrm{k}=1$ to $\mathrm{k}=17$. These values of " $\mathrm{k}$ " were generated using an $x \log (\mathrm{x}) \mathrm{scale}$, starting at $x=2$ and incrementing. The " $k$ " values used in this, extend as seen in Table B.1. This table also offers a view at which data sets have been run for each classifier and value of K. The significance of k-values has been described $\$ 2.1$.

In order to determine the efficacy of these techniques for filtering, we define a number "T" which represents the number of predictions we are narrowing the selection to. As described in $\$ 2.1$, the "T" value used in this thesis is $\mathrm{T}=5$. Therefore, a successful filtering is considered to be a case where the correct prediction was within the top five predictions offered by the classifier.

\subsection{Preprocessing}

Preprocessing of the raw XML data is done in several steps. A series of scripts perform the following tasks:

- Strip out any record which only occurs once in the data set. This step is performed in order to eliminate any chance of predicting access for a JAN which will only ever occur once within the workflow. This is done post-hoc for this data, but could easily be done on-the-fly in an actual implementation of a WPCKAM via manipulation of the training sets.

- Strip out any record which is not part of the whitelist seen in Table B.4. This eliminates any- 


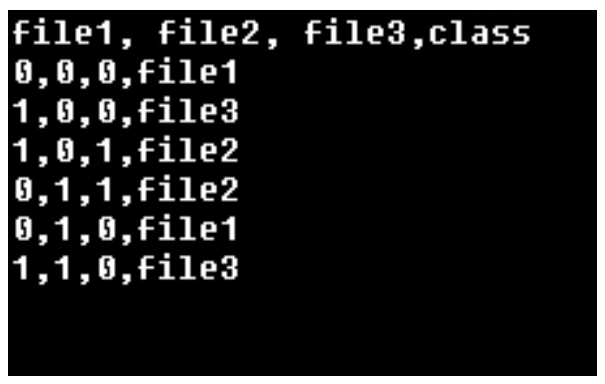

Figure 5.1: Example Comma Separated Values File

thing that is not considered a document or a programming source code file. These extensions were gathered from [1]. This action is performed so that we are specifically searching for knowledge-work-related patterns.

- Convert each record into an MD5 hash. The purpose of this step is simply to avoid any sensitive user data from being displayed in plaintext.

The final step of the preprocessing is to construct a CSV ${ }^{1}$ from the collected data. These CSV files consist of records (lines) and fields (each value, separated by commas). A small sample CSV file is shown in Figure 5.1. The first line of this file is a listing of the attribute names. Every other record is a series of digits, then the attribute value. This attribute value represents the filename. The digits represent whether the various files were seen in the last "k" records of the training set; a "1" indicates that the corresponding attribute value was seen within the last $\mathrm{k}$ units, and a "0" indicates that it was not. Several values of "k" were used for this experimental setup, shown in Table B.1. Note that the example CSV file in Figure 5.1 is constructed using a k value of "2".

\subsection{Results}

This section offers description of charting for global results.

\footnotetext{
${ }^{1}$ Comma-separated values
} 
- Table 5.1 shows various statistical data for predictions across all datasets for values of $\mathrm{k}$, as well as a box plot of this data.

- Table 5.2 shows various statistical data for predictions across all datasets for classifiers, as well as a box plot of this data.

- Table 5.3 shows various statistical data for predictions across all datasets for each dataset, as well as a box plot of this data.

- Table 5.4 shows various statistical data for filtering across all datasets for values of $\mathrm{k}$, as well as a box plot of this data.

- Table 5.5 shows various statistical data for filtering across all datasets for classifiers, as well as a box plot of this data.

- Table 5.6 shows various statistical data for filtering across all datasets for each dataset, as well as a box plot of this data.

- Table 5.7 demonstrates prediction counts across all values of $\mathrm{k}$, as well as prediction means.

- Table 5.8 demonstrates prediction counts across all classifiers, as well as prediction means.

- Table 5.9 demonstrates prediction counts across all datasets, as well as prediction means.

- Table 5.10 demonstrates filter counts across all values of $\mathrm{k}$, as well as filtering means.

- Table 5.11 demonstrates filter counts across all classifiers, as well as filtering means.

- Table 5.12 demonstrates filter counts across all datasets, as well as filtering means. 


\begin{tabular}{|c|c|c|c|c|c|c|}
\hline \multicolumn{7}{|c|}{ Results } \\
\hline k-value & $\min$ & $\mathrm{q} 1$ & median & q3 & $\max$ & Quartile \\
\hline 1 & 0 & 16 & 52 & 67 & 78 & $\bullet$ \\
\hline 3 & 0 & 29 & 44 & 62 & 80 & $\longrightarrow$ \\
\hline 6 & 0 & 25 & 52.5 & 63 & 79 & $\mathbf{0}$ \\
\hline 8 & 0 & 25 & 49.5 & 59 & 79 & $\longrightarrow$ \\
\hline 11 & 0 & 20 & 42.5 & 58 & 77 & $\bullet$ \\
\hline 14 & 0 & 19 & 36.5 & 52 & 69 & $\bullet+$ \\
\hline
\end{tabular}

Table 5.1: Prediction success rates by k-value for all datasets

\begin{tabular}{|c|c|c|c|c|c|c|}
\hline \multicolumn{7}{|c|}{ Results } \\
\hline Classifier & $\min$ & $\mathrm{q} 1$ & median & q3 & $\max$ & Quartile \\
\hline Hyperpipes & 8 & 23 & 33.5 & 56.5 & 70 & $\rightarrow \quad+$ \\
\hline Naive Bayes & 6 & 46 & 58 & 67 & 79 & $+\bullet$ \\
\hline OneR & 0 & 11 & 14.5 & 33 & 63 & $0 \quad 1$ \\
\hline $\mathrm{J} 48$ & 6 & 37 & 56.5 & 69.5 & 80 & -10 \\
\hline LWL & 5 & 25 & 48 & 59.5 & 78 & $a-$ \\
\hline
\end{tabular}

Table 5.2: Prediction success rates by classifier for all datasets

\begin{tabular}{|c|c|c|c|c|c|c|}
\hline \multicolumn{7}{|c|}{ Results } \\
\hline Dataset & $\min$ & $\mathrm{q} 1$ & median & q3 & $\max$ & Quartile \\
\hline Set 1 & 10 & 21 & 33 & 41 & 53 & $\bullet-1$ \\
\hline Set 2 & 38 & 56 & 59 & 63 & 67 & - \\
\hline Set 3 & 27 & 48 & 54 & 63 & 68 & $+\bullet-$ \\
\hline Set 4 & 14 & 30 & 62.5 & 75 & 77 & 10 \\
\hline Set 5 & 1 & 5.5 & 8 & 11.5 & 18 & - \\
\hline Set 6 & 0 & 6 & 20 & 35.5 & 55 & $\bullet \quad \mid$ \\
\hline Set 7 & 12 & 52 & 56.5 & 62 & 68 & $\bullet$ \\
\hline Set 8 & 10 & 24 & 39 & 51 & 60 & $\bullet$ \\
\hline Set 9 & 21 & 33 & 48.5 & 70 & 75 & $\rightarrow$ \\
\hline Set 10 & 14 & 34 & 58.5 & 77 & 80 & $-1 \bullet$ \\
\hline
\end{tabular}

Table 5.3: Prediction success rates by dataset for all datasets 


\begin{tabular}{|l|r|r|r|r|r|r|r|}
\multicolumn{1}{l|}{ k-value } & \multicolumn{1}{c|}{$\min$} & \multicolumn{1}{c|}{$\mathrm{q} 1$} & median & $\mathrm{q} 3$ & $\max$ & Quartile & \\
\hline 1 & 24 & 74.5 & 93 & 99 & 100 & & - \\
\hline 3 & 27 & 73.5 & 94 & 100 & 100 & & $\bullet$ \\
\hline 6 & 23 & 82 & 94 & 99 & 100 & & - \\
\hline 8 & 25 & 85.5 & 94.5 & 99 & 100 & & - \\
\hline 11 & 25 & 83.5 & 94.5 & 99.5 & 100 & & - \\
\hline 14 & 22 & 75.5 & 91 & 99 & 100 & & $-\bullet$ \\
\hline
\end{tabular}

Table 5.4: Filtering success rates by k-value for all datasets

\begin{tabular}{|l|r|r|r|r|r|r|r|} 
& \multicolumn{9}{c|}{ Results } \\
Classifier & min & \multicolumn{1}{c|}{ 1 } & median & \multicolumn{1}{c|}{ 3} & $\max$ & Quartile & \\
\hline Hyperpipes & 24 & 79 & 94 & 98 & 100 & & - \\
\hline Naive Bayes & 27 & 86 & 94 & 98 & 100 & & - \\
\hline J48 & 27 & 86.5 & 99 & 100 & 100 & & - \\
\hline LWL & 22 & 66 & 89 & 95 & 100 & & - \\
\hline
\end{tabular}

Table 5.5: Filtering success rates by classifier for all datasets

\begin{tabular}{|c|c|c|c|c|c|c|c|}
\hline \multicolumn{8}{|c|}{ Results } \\
\hline Dataset & $\min$ & $\mathrm{q} 1$ & median & q3 & $\max$ & Quartile & \\
\hline Set 1 & 64 & 72.5 & 81.5 & 86 & 100 & I & $\rightarrow$ \\
\hline Set 2 & 100 & 100 & 100 & 100 & 100 & I & $\bullet$ \\
\hline Set 3 & 87 & 92 & 94 & 99 & 100 & I & - \\
\hline Set 4 & 66 & 88 & 93.5 & 96 & 100 & I & $\bullet$ \\
\hline Set 5 & 22 & 26.5 & 29.5 & 74 & 96 & $0 \quad \mid$ & \\
\hline Set 6 & 27 & 33 & 63 & 81.5 & 90 & -1 & 5 \\
\hline Set 7 & 98 & 98 & 99 & 100 & 100 & I & $\bullet$ \\
\hline Set 8 & 69 & 86.5 & 91.5 & 94 & 100 & I & $\bullet$ \\
\hline Set 9 & 91 & 93.5 & 98 & 100 & 100 & I & $\bullet$ \\
\hline Set 10 & 70 & 89 & 96 & 97 & 100 & I & $-\bullet$ \\
\hline
\end{tabular}

Table 5.6: Filtering success rates by dataset for all datasets 


\begin{tabular}{|l|r|r|l|} 
k-value & Successes & Failures & Average of Success Rates \\
\hline 1 & 15850 & 23115 & $44.6 \%$ \\
\hline 3 & 15471 & 23494 & $42.74 \%$ \\
\hline 6 & 16352 & 22613 & $44.78 \%$ \\
\hline 8 & 15728 & 23237 & $43.02 \%$ \\
\hline 11 & 14130 & 24835 & $39.46 \%$ \\
\hline 14 & 12558 & 26407 & $35.32 \%$ \\
\hline
\end{tabular}

Table 5.7: Prediction data with number of predictions by k-value for all datasets

\begin{tabular}{|c|c|c|c|}
\hline Classifier & Successes & Failures & Average of Success Rates \\
\hline Hyperpipes & 15028 & 31682 & $36.45 \%$ \\
\hline Naive Bayes & 24105 & 22665 & $54.02 \%$ \\
\hline OneR & 8939 & 37831 & $23.05 \%$ \\
\hline J48 & 23455 & 23315 & $51.78 \%$ \\
\hline LWL & 18562 & 28208 & $42.97 \%$ \\
\hline
\end{tabular}

Table 5.8: Prediction data with number of predictions by classifier for all datasets

\begin{tabular}{|l|r|r|l|} 
& \multicolumn{3}{|l|}{} \\
Dataset & Successes & Failures & Average of Success Rates \\
\hline Set 1 & 5251 & 11759 & $31 \%$ \\
\hline Set 2 & 3208 & 2522 & $55.9 \%$ \\
\hline Set 3 & 1938 & 1782 & $52.1 \%$ \\
\hline Set 4 & 8471 & 7369 & $53.53 \%$ \\
\hline Set 5 & 2747 & 29443 & $8.47 \%$ \\
\hline Set 6 & 7246 & 23984 & $23.17 \%$ \\
\hline Set 7 & 15055 & 14135 & $51.53 \%$ \\
\hline Set 8 & 12493 & 22097 & $36.13 \%$ \\
\hline Set 9 & 15851 & 15529 & $50.47 \%$ \\
\hline Set 10 & 17829 & 15081 & $54.23 \%$ \\
\hline
\end{tabular}

Table 5.9: Prediction data with number of predictions by dataset for all datasets 


\begin{tabular}{|l|r|r|l|} 
k-value & Successes & Failures & Average of Success Rates \\
\hline 1 & 24323 & 6847 & $82.02 \%$ \\
\hline 3 & 26014 & 5156 & $86.37 \%$ \\
\hline 6 & 26108 & 5062 & $86.57 \%$ \\
\hline 8 & 25925 & 5245 & $86.1 \%$ \\
\hline 11 & 26352 & 4818 & $87 \%$ \\
\hline 14 & 24746 & 6424 & $82.52 \%$ \\
\hline
\end{tabular}

Table 5.10: Filtering data with number of predictions by k-value for all datasets

\begin{tabular}{|c|c|c|c|}
\hline Classifier & Successes & Failures & Average of Success Rates \\
\hline Hyperpipes & 37602 & 9108 & $83.95 \%$ \\
\hline Naive Bayes & 40941 & 5829 & $88.85 \%$ \\
\hline $\mathrm{J} 48$ & 41839 & 4931 & $91.82 \%$ \\
\hline LWL & 33086 & 13684 & $75.78 \%$ \\
\hline
\end{tabular}

Table 5.11: Filtering data with number of predictions by classifier for all datasets

\begin{tabular}{|c|c|c|c|}
\hline Dataset & Successes & Failures & Average of Success Rates \\
\hline Set 1 & 10965 & 2643 & $80.62 \%$ \\
\hline Set 2 & 4572 & 0 & $100 \%$ \\
\hline Set 3 & 2820 & 156 & $94.75 \%$ \\
\hline Set 4 & 11595 & 1077 & $91.46 \%$ \\
\hline Set 5 & 11860 & 13892 & $46.04 \%$ \\
\hline Set 6 & 14897 & 10087 & $59.63 \%$ \\
\hline Set 7 & 23174 & 178 & $99.04 \%$ \\
\hline Set 8 & 24601 & 3071 & $88.96 \%$ \\
\hline Set 9 & 24379 & 725 & $97 \%$ \\
\hline Set 10 & 24605 & 1723 & $93.5 \%$ \\
\hline
\end{tabular}

Table 5.12: Filtering data with number of predictions by dataset for all datasets 


\subsection{Classification}

For all of the tested classifiers, training sets are required. We begin by acquiring a basis of "N" records which will be used as the initial training set. The first " $\mathrm{N}$ " records will therefore not be a part of the predictive algorithm. The algorithm then attempts to classify record "R", the first record after the basis "N". The classifier's prediction is then compared to "R", and the number of correct and incorrect classifications is tallied up. At this point, the algorithm also tallies up the number of "correctly filtered" predictions; we consider a record "correctly filtered" if the actual record "R" was within the classifier's top five predictions.

After the results are recorded, the algorithm adds $\mathrm{R}$ to the training set, and moves on to classify $\mathrm{R}+1$. This process continues until the entire data set has been classified.

\subsubsection{Hyperpipes}

Hyperpipes's performance as a predictor was poor, outperforming only OneR with respect to the other classifiers across all data sets. While its performance as a filterer could be considered adequate, it was still outperformed by all of the other classifiers across all data sets.

\subsubsection{Naive Bayes Classification}

Naive Bayes's performance as a predictor was superb, outperforming all over the other tested classifiers. Its performance as a filterer was adequate, as it was outperformed only by J48.

\subsubsection{J48 Classification}

This research's implementation of J48 had the following attributes:

- Confidence threshold for pruning: 0.25

- Minimum number of instances per leaf: 2 
- Number of folds for reduced error pruning: 3

J48's performance as a predictor was adequate, outperforming OneR, Hyperpipes, and LWL. Its performance as a filterer was superb, as it outperformed all other tested classifiers.

\subsubsection{LWL Classification}

This research's implementation of LWL had the following attributes:

- Inputs are normalized.

- All neighbors are used to set kernel bandwidth.

- Weighting kernel is linear.

LWL's performance as a predictor was adequate, outperforming OneR and Hyperpipes, but being outperformed by J48 and Naive Bayes. As a filterer, LWL performed well, but was outperformed by every other tested classifier except Hyperpipes.

\subsubsection{OneR Classification}

This research's implementation of OneR had the following attributes:

- Minimum bucket size is 6 .

As stated in $\$ 3.6 .5$, OneR is a classifier that is not typically expected to perform well. It is therefore no surprise that OneR was outperformed by all of the other tested classifiers when used as a predictor. Due to the nature of OneR's implementation, data could not be collected for its efficacy as a predictor. 


\subsection{Analysis}

Statistical analysis and graphing was done using ezANOVA [32]'s implementation of ANOVA. ANOVA stands for Analysis of Variance, and, in this case, consists of a "repeated measures" design in that it uses the same subjects repeatedly. ANOVA's F-Test seeks to compare the ratio of explained variance to unexplained variance.

For prediction, the following p-values were calculated:

- k-value: $\mathrm{p}=0.159987$

- Classifier: $\mathrm{p}=0.000001$

For filtering, the following p-values were calculated:

- k-value: $\mathrm{p}=0.079020$

- Classifier: $\mathrm{p}=0.001818$

In terms of the hypotheses ${ }^{2}$, these results indicate:

- We fail to reject null hypothesis 1 at the 0.05 level of significance. The k-value is not a significant factor for predictions.

- We reject null hypothesis 2 at the 0.05 level of significance. The classifier is a significant factor for predictions.

- We fail to reject null hypothesis 3 at the 0.05 level of significance. The k-value is not a significant factor for filtering.

- We reject null hypothesis 4 at the 0.05 level of significance. The classifier is a significant factor for filtering.

\footnotetext{
${ }^{2}$ See $\$ 2.2$
} 


\subsection{Summary}

This chapter has consisted of an in-depth look at the experimental portion of this thesis. Information was given about how data was collected, as well as how the data was preprocessed and analyzed. The importance of values of "k" was explained, as well as how these k-values were generated.

This chapter has also directly presented the most interesting and pertinent data for this research. A statistical analysis of this data has been given. 


\section{Chapter 6}

\section{Conclusions}

This chapter provides conclusions garnered from the resulting data found in Chapter 5 .

This chapter is therefore divided as follows:

- $\$ 6.1$ reviews the hypotheses laid out in $\$ 2.2$ and offers conclusions based on the ANOVA tests performed on $\$ 5.3$ and shown in $\$ 5.5$

- $\$ 6.2$ explores how the specific results of this research could be applied to producing a knowledge advantage, with respect to the concepts detailed in $\$ 4.4$.

- A future work section is provided in $\$ 6.3$, which offers an overview of the possible "next steps" that this research could take in the pursuit of the implementation of a fully realized KAM.

\subsection{Hypotheses}

As seen in $\$ 5.5$, the following conclusions were reached through ANOVA testing:

- The $\mathrm{k}$-value is not a significant factor for predictions.

- The classifier is a significant factor for predictions. 
- The k-value is not a significant factor for filtering.

- The classifier is a significant factor for filtering.

One interesting result is that the choice of k-value does not appear to be a factor in both prediction and filtering. However, upon examining the charting in $\$ 5.3$, it becomes obvious that this is for two separate reasons. In predictions, the data indicates that the $\mathrm{k}$-value is not a factor because, across classifiers, they universally perform poorly. However, in filtering, the data indicates that the $\mathrm{k}$-value is not a factor because they universally perform very well.

The statistical analysis indicates that classifiers are a significant factor for both prediction and filtering. We see in Table 5.8 that, on average, Naive Bayes and J48 were the only classifiers to perform with higher than 50 percent accuracy. Likewise in Table 5.11, we find that Naive Bayes and $\mathrm{J} 48$ are the highest performing classifiers, though all classifiers (aside from OneR) performed with above 75 percent accuracy. Regardless, if this workflow prediction concept were to be used for a KAM, it follows that more testing would be useful to determine if there are classifiers which prove superior to the ones tested in this research.

Finally, we see in Table 5.12 that a few sets of data appear significantly less efficient for filtering. This suggests that certain patterns of file access exist which are less copacetic with the type of prediction-filtering-via-classification-learning which is presented within this research. Put simply, some workflow patterns do not lend themselves to workflow analysis. For sets with such patterns, it might become more prudent to place more importance on alternate forms of context identification, such as semantic analysis.

\subsection{How the Advantage is Gained}

As seen in $\$ 4.4$ and its subsections, we have explored many areas in which an advantage might be gained through the use of this technology. Now that we have empirically shown the efficacy of 
filtering over prediction, each of these concepts can be reexamined with the promising results of filtering in mind.

\subsubsection{Context Awareness}

As discussed in $\$ 4.4 .1$, context awareness is necessary for any KAM and workflow prediction’s main goal is to assist in this awareness of context. With filtering, a WPCKAM may be able to identify contextually similar JANs. Since this thesis has empirically shown that filtering works, one might start by tallying up occurences of JANs appearing with other JANs in the sets of "top T" predictions.

Contextually similar JANs might be defined as JANs who appear concurrently within the "top T" predictions beyond a certain threshold of times. Once an awareness of conextual similarity is established, we might then proceed to use this information for any task which requires context awareness. Furthermore, we might semantically analyze the JANs which have been identified as contextually similar through workflow prediction; this combination of methods might lead us to a more robust and accurate context prediction mechanic.

\subsubsection{Presenting Predicted Files}

Because filtering has been shown to work, prediction presentation could be accomplished without any further research. This thesis has provided an agent capable of filtering file accesses with a high degree of success; therefore, a tool could be created which simply pushes these "top T" predictions to a pane or folder as described in $\$ 4.4 .2$. This is a simple example of a knowledge advantage which could be quickly and easily be created using only the methods described in this research. 


\subsubsection{Measuring JAN Importance}

Using filtering, JAN importance could be assessed by tallying up how frequently various JANs appear in the "top T" predicted results. Various thresholds of importance could be arbitrarily set, and once a JAN crosses one of these thresholds, it might be selected for semantic analysis. As described in $\$ 4.4 .3$, we might then have the discovery agent search for similar JANs and place them into the user's personal ontology.

\subsection{Future Work}

A great abundance of future work waits to be explored in the area of KAM research. For future work pertaining specifically to work-patterns, however, there are some interesting research avenues that the results of this thesis point towards. These include:

- Test more learning techniques. Five classifiers were tested in this thesis; many other classification techniques exist. Furthermore, learning techniques such as clustering could be employed and examined for efficacy.

- Examine other types of workflow patterns aside from file usage data, using techniques similar to those used in this thesis. One example would be applying classification techniques to web usage data.

- Combine the work-pattern centric approach to context prediction with other context identification techniques. Algorithms which perform semantic analysis, for example, could be augmented by examining the filtered predictions provided by workflow prediction.

- Examine these concepts as they pertain to predicting application use in addition to file usage, such as seen in [26]. 


\section{Appendix A}

\section{Reproducing the Experiments}

This appendix provides information on how to replicate the described experiments via the same methods used in this thesis. Various scripts and instructions are provided. Sample data is not provided, due to privacy concerns; however, specific instructions on how to collect data are shown.

\section{A.1 Acquiring the Software}

The scripts used to preprocess the data are available at the WVU'S CERC server. It is suggested that these tools be used on a Unix system, as some of the preprocessing is done using Unix command line utilities. A zip file of all the software is available at cerc.wvu.edu/kam/wpckam.zip. This contains:

- Two pdf files, "auditingbegin.pdf" and "auditingend.pdf." These are instructions on how to start and stop file usage auditing via Windows 7 Pro.

- One PHP script, "auditing.php". This will take in an XML file and print out filenames in sequence. Output should be sent to a text file.

- One extensionless script, "preprocess". This performs some preliminary whitelisting on the 
output file generated with auditing.php.

- Two Java sourcecode files, FileUsageDataAnalyzer.java and Main.java. These should be jarred up and run. Using the preprocessed file, they apply more preprocessing, generate MD5 hashed CSV files, and perform classifications in sequence.

- Two jar files, "commons-lang3-3.1jar" and "weka.jar". These are included for convenience, and are necessary for running the Java files listed above.

\section{A.2 Obtaining Data}

After running the provided Java code, a file will be generated based on the name of the dataset file, the value of $\mathrm{k}$ used, and the classifier used. The first line of this file will consist of the number of successful predictions using that classifier, then a comma, then the number of unsuccessful predictions using that classifier. The second line of this file will consist of the number of successful filterings using a "T" value of 5, then a comma, then the number of unsuccessful filterings using that classifier.

From the resultant data, whatever statistical analysis you deem appropriate can be performed.

\section{A.3 Running your Experiments}

After XML datasets have been acquired using the instructions found in the two hosted pdf files described in \$A.1, classification can begin. The provided suite of tools can be used on a Unix system as follows:

- Run "auditing.php" from the command line, using the syntax "php auditing.php filename.XML CSV". Send the output of this command to an extensionless text file, "output". 
- Run "preprocess" from the command line. This will generate a preprocessed file, outputFinal.txt.

- After jarring the sourcecode files, run the jar with the outputFinal file in the same folder.

- The first command line argument is the name of the dataset file.

- The second command line argument is the number of records to use as the basis training set.

- The third command line argument is the " $\mathrm{x}$ " in the xlogx scale used to generate the $\mathrm{k}$-value.

- The fourth command line argument is the classifier: options are "PIPES", "BAYES", "LWL", "J48", and "ONER". It is also important to provide the Java virtual machine with enough memory. Therefore, a sample command line run would be: "java -Xms256m -Xmx1500m -jar Analyzer.jar outputFinal 1002 PIPES”. 


\section{Appendix B}

\section{Raw Data and Charts}

\section{B.1 Various Charts}

Table B.2: Prediction success rates by all factors for all datasets

\begin{tabular}{|c|c|c|c|} 
& Successes & Failures & Average of Success Rates \\
\hline Set 1 k1 Hyperpipes & 173 & 394 & $31 \%$ \\
\hline Set 1 k1 Naive Bayes & 272 & 295 & $48 \%$ \\
\hline Set 1 k1 OneR & 62 & 505 & $11 \%$ \\
\hline Set 1 k1 J48 & 210 & 357 & $37 \%$ \\
Continuing next page & & &
\end{tabular}

\begin{tabular}{|l|r|r|r|r|r|r|l|}
\multicolumn{8}{|c|}{-values } \\
Classifiers & 1 & 3 & 6 & 8 & 11 & 14 & 17 \\
\hline Hyperpipes & $1-5$ & $1-5$ & $1-5$ & $1-5$ & $1-5$ & $1-5$ & $1-5$ \\
Naive Bayes & $1-5$ & $1-5$ & $1-5$ & $1-5$ & $1-5$ & $1-5$ & $1-5$ \\
OneR & $1-5$ & $1-5$ & $1-5$ & $1-5$ & $1-5$ & $1-5$ & $1-5$ \\
J48 & $1-5$ & $1-5$ & $1-5$ & $1-5$ & $1-5$ & $1-5$ & $1-5$ \\
LWL & $1-5$ & $1-5$ & $1-5$ & $1-5$ & $1-5$ & $1-5$ & $1-5$
\end{tabular}

Table B.1: Values of $\mathrm{k}$ 


\begin{tabular}{|c|c|c|c|} 
& Successes & Failures & Average of Success Rates \\
\hline Set 1 k1 LWL & 231 & 336 & $41 \%$ \\
\hline Set 1 k3 Hyperpipes & 175 & 392 & $31 \%$ \\
\hline Set 1 k3 Naive Bayes & 236 & 331 & $42 \%$ \\
\hline Set 1 k3 OneR & 61 & 506 & $11 \%$ \\
\hline Set 1 k3 J48 & 210 & 357 & $37 \%$ \\
\hline Set 1 k3 LWL & 168 & 399 & $30 \%$ \\
\hline Set 1 k6 Hyperpipes & 219 & 348 & $39 \%$ \\
\hline Set 1 k6 Naive Bayes & 300 & 267 & $53 \%$ \\
\hline Set 1 k6 OneR & 62 & 505 & $11 \%$ \\
\hline Set 1 k6 J48 & 241 & 326 & $43 \%$ \\
\hline Set 1 k6 LWL & 196 & 371 & $35 \%$ \\
\hline Set 1 k8 Hyperpipes & 202 & 365 & $36 \%$ \\
\hline Set 1 k8 Naive Bayes & 290 & 277 & $51 \%$ \\
\hline Set 1 k8 OneR & 60 & 507 & $11 \%$ \\
\hline Set 1 k8 J48 & 233 & 334 & $41 \%$ \\
\hline Set 1 k8 LWL & 186 & 381 & $33 \%$ \\
\hline Set 1 k11 Hyperpipes & 151 & 416 & $27 \%$ \\
\hline Set 1 k11 Naive Bayes & 185 & 382 & $33 \%$ \\
\hline Set 1 k11 OneR & 61 & 506 & $23 \%$ \\
\hline Set 1 k11 J48 & 166 & 401 & \\
\hline Set 1 k11 LWL & 154 & 413 & 434 \\
\hline Set 1 k14 Hyperpipes & 133 & 434 & \\
\hline Continuing next page & & & $39 \%$ \\
\hline
\end{tabular}




\begin{tabular}{|c|c|c|c|}
\hline & Successes & Failures & Average of Success Rates \\
\hline Set 1 k14 Naive Bayes & 251 & 316 & $44 \%$ \\
\hline Set 1 k14 OneR & 59 & 508 & $10 \%$ \\
\hline Set $1 \mathrm{k} 14 \mathrm{~J} 48$ & 197 & 370 & $35 \%$ \\
\hline Set $1 \mathrm{k} 14 \mathrm{LWL}$ & 107 & 460 & $19 \%$ \\
\hline Set 2 k1 Hyperpipes & 70 & 113 & $38 \%$ \\
\hline Set 2 k1 Naive Bayes & 129 & 64 & $67 \%$ \\
\hline Set 2 k1 OneR & 121 & 72 & $63 \%$ \\
\hline Set $2 \mathrm{k} 1 \mathrm{~J} 48$ & 126 & 67 & $65 \%$ \\
\hline Set $2 \mathrm{k} 1 \mathrm{LWL}$ & 129 & 64 & $67 \%$ \\
\hline Set 2 k3 Hyperpipes & 69 & 114 & $38 \%$ \\
\hline Set 2 k3 Naive Bayes & 115 & 78 & $60 \%$ \\
\hline Set 2 k3 OneR & 112 & 81 & $58 \%$ \\
\hline Set $2 \mathrm{k} 3 \mathrm{~J} 48$ & 110 & 83 & $57 \%$ \\
\hline Set $2 \mathrm{k} 3 \mathrm{LWL}$ & 116 & 77 & $60 \%$ \\
\hline Set 2 k6 Hyperpipes & 69 & 114 & $38 \%$ \\
\hline Set 2 k6 Naive Bayes & 127 & 66 & $66 \%$ \\
\hline Set 2 k6 OneR & 118 & 75 & $61 \%$ \\
\hline Set 2 k6 J48 & 122 & 71 & $63 \%$ \\
\hline Set 2 k6 LWL & 122 & 71 & $63 \%$ \\
\hline Set 2 k8 Hyperpipes & 69 & 114 & $38 \%$ \\
\hline Set 2 k8 Naive Bayes & 115 & 78 & $60 \%$ \\
\hline Set $2 \mathrm{k} 8$ OneR & 112 & 81 & $58 \%$ \\
\hline
\end{tabular}




\begin{tabular}{|c|c|c|c|} 
& Successes & Failures & Average of Success Rates \\
\hline Set 2 k8 J48 & 114 & 79 & $59 \%$ \\
\hline Set 2 k8 LWL & 113 & 80 & $59 \%$ \\
\hline Set 2 k11 Hyperpipes & 69 & 114 & $38 \%$ \\
\hline Set 2 k11 Naive Bayes & 113 & 80 & $59 \%$ \\
\hline Set 2 k11 OneR & 109 & 84 & $56 \%$ \\
\hline Set 2 k11 J48 & 112 & 81 & $58 \%$ \\
\hline Set 2 k11 LWL & 113 & 80 & $59 \%$ \\
\hline Set 2 k14 Hyperpipes & 70 & 113 & $38 \%$ \\
\hline Set 2 k14 Naive Bayes & 111 & 82 & $58 \%$ \\
\hline Set 2 k14 OneR & 109 & 84 & $56 \%$ \\
\hline Set 2 k14 J48 & 111 & 82 & $58 \%$ \\
\hline Set 2 k14 LWL & 113 & 80 & $59 \%$ \\
\hline Set 3 k1 Hyperpipes & 75 & 49 & $60 \%$ \\
\hline Set 3 k1 Naive Bayes & 84 & 40 & $68 \%$ \\
\hline Set 3 k1 OneR & 37 & 87 & $30 \%$ \\
\hline Set 3 k1 J48 & 78 & 46 & $63 \%$ \\
\hline Set 3 k1 LWL & 82 & 42 & $66 \%$ \\
\hline Set 3 k3 Hyperpipes & 78 & 46 & $63 \%$ \\
\hline Set 3 k3 Naive Bayes & 82 & 42 & $53 \%$ \\
\hline Set 3 k3 OneR & 38 & 86 & $58 \%$ \\
\hline Set 3 k3 J48 & 66 & 58 & 59 \\
\hline Set 3 k3 LWL & 65 & 59 & \\
\hline Continuing next page & & & $59 \%$ \\
\hline
\end{tabular}




\begin{tabular}{|c|c|c|c|} 
& Successes & Failures & Average of Success Rates \\
\hline Set 3 k6 Hyperpipes & 77 & 47 & $62 \%$ \\
\hline Set 3 k6 Naive Bayes & 80 & 44 & $65 \%$ \\
\hline Set 3 k6 OneR & 39 & 85 & $31 \%$ \\
\hline Set 3 k6 J48 & 66 & 58 & $53 \%$ \\
\hline Set 3 k6 LWL & 60 & 64 & $48 \%$ \\
\hline Set 3 k8 Hyperpipes & 76 & 48 & $61 \%$ \\
\hline Set 3 k8 Naive Bayes & 78 & 46 & $63 \%$ \\
\hline Set 3 k8 OneR & 37 & 87 & $30 \%$ \\
\hline Set 3 k8 J48 & 68 & 56 & $55 \%$ \\
\hline Set 3 k8 LWL & 59 & 65 & $48 \%$ \\
\hline Set 3 k11 Hyperpipes & 81 & 43 & $65 \%$ \\
\hline Set 3 k11 Naive Bayes & 74 & 50 & $60 \%$ \\
\hline Set 3 k11 OneR & 35 & 89 & $28 \%$ \\
\hline Set 3 k11 J48 & 63 & 61 & $51 \%$ \\
\hline Set 3 k11 LWL & 59 & 65 & $48 \%$ \\
\hline Set 3 k14 Hyperpipes & 78 & 46 & $63 \%$ \\
\hline Set 3 k14 Naive Bayes & 68 & 56 & $55 \%$ \\
\hline Set 3 k14 OneR & 34 & 90 & $27 \%$ \\
\hline Set 3 k14 J48 & 64 & 60 & $52 \%$ \\
\hline Set 3 k14 LWL & 57 & 67 & $75 \%$ \\
\hline Set 4 k1 Hyperpipes & 367 & 161 & 134 \\
\hline Set 4 k1 Naive Bayes & 394 & & \\
\hline Continuing next page & & & $50 \%$ \\
\hline
\end{tabular}




\begin{tabular}{|c|c|c|c|} 
& Successes & Failures & Average of Success Rates \\
\hline Set 4 k1 OneR & 83 & 445 & $16 \%$ \\
\hline Set 4 k1 J48 & 398 & 130 & $75 \%$ \\
\hline Set 4 k1 LWL & 404 & 124 & $77 \%$ \\
\hline Set 4 k3 Hyperpipes & 344 & 184 & $65 \%$ \\
\hline Set 4 k3 Naive Bayes & 404 & 124 & $77 \%$ \\
\hline Set 4 k3 OneR & 78 & 450 & $15 \%$ \\
\hline Set 4 k3 J48 & 398 & 130 & $75 \%$ \\
\hline Set 4 k3 LWL & 333 & 195 & $63 \%$ \\
\hline Set 4 k6 Hyperpipes & 331 & 197 & $63 \%$ \\
\hline Set 4 k6 Naive Bayes & 399 & 129 & $76 \%$ \\
\hline Set 4 k6 OneR & 77 & 451 & $15 \%$ \\
\hline Set 4 k6 J48 & 396 & 132 & $75 \%$ \\
\hline Set 4 k6 LWL & 301 & 227 & $57 \%$ \\
\hline Set 4 k8 Hyperpipes & 330 & 198 & $62 \%$ \\
\hline Set 4 k8 Naive Bayes & 385 & 143 & $73 \%$ \\
\hline Set 4 k8 OneR & 76 & 452 & $14 \%$ \\
\hline Set 4 k8 J48 & 394 & 134 & $75 \%$ \\
\hline Set 4 k8 LWL & 292 & 236 & $55 \%$ \\
\hline Set 4 k11 Hyperpipes & 326 & 202 & $62 \%$ \\
\hline Set 4 k11 Naive Bayes & 374 & 154 & $73 \%$ \\
\hline Set 4 k11 OneR & 74 & 454 & $14 \%$ \\
\hline Set 4 k11 J48 & 385 & 143 & \\
\hline Continuing next page & & & \\
\hline
\end{tabular}




\begin{tabular}{|c|c|c|c|}
\hline & Successes & Failures & Average of Success Rates \\
\hline Set $4 \mathrm{k} 11 \mathrm{LWL}$ & 263 & 265 & $50 \%$ \\
\hline Set 4 k14 Hyperpipes & 160 & 368 & $30 \%$ \\
\hline Set 4 k14 Naive Bayes & 219 & 309 & $41 \%$ \\
\hline Set 4 k14 OneR & 78 & 450 & $15 \%$ \\
\hline Set $4 \mathrm{k} 14 \mathrm{~J} 48$ & 274 & 254 & $52 \%$ \\
\hline Set 4 k14 LWL & 134 & 394 & $25 \%$ \\
\hline Set 5 k1 Hyperpipes & 86 & 987 & $8 \%$ \\
\hline Set 5 k1 Naive Bayes & 159 & 914 & $15 \%$ \\
\hline Set $5 \mathrm{k} 1$ OneR & 15 & 1058 & $1 \%$ \\
\hline Set $5 \mathrm{k} 1 \mathrm{~J} 48$ & 66 & 1007 & $6 \%$ \\
\hline Set 5 k1 LWL & 72 & 1001 & $7 \%$ \\
\hline Set 5 k3 Hyperpipes & 106 & 967 & $10 \%$ \\
\hline Set 5 k 3 Naive Bayes & 115 & 958 & $11 \%$ \\
\hline Set $5 \mathrm{k} 3$ OneR & 15 & 1058 & $1 \%$ \\
\hline Set $5 \mathrm{k} 3 \mathrm{~J} 48$ & 82 & 991 & $8 \%$ \\
\hline Set 5 k3 LWL & 57 & 1016 & $5 \%$ \\
\hline Set 5 k6 Hyperpipes & 175 & 898 & $16 \%$ \\
\hline Set 5 k6 Naive Bayes & 192 & 881 & $18 \%$ \\
\hline Set 5 k6 OneR & 15 & 1058 & $1 \%$ \\
\hline Set $5 \mathrm{k} 6 \mathrm{~J} 48$ & 111 & 962 & $10 \%$ \\
\hline Set $5 \mathrm{k} 6 \mathrm{LWL}$ & 65 & 1008 & $6 \%$ \\
\hline Set 5 k8 Hyperpipes & 165 & 908 & $15 \%$ \\
\hline
\end{tabular}




\begin{tabular}{|c|c|c|c|}
\hline & Successes & Failures & Average of Success Rates \\
\hline Set 5 k8 Naive Bayes & 186 & 887 & $17 \%$ \\
\hline Set $5 \mathrm{k} 8$ OneR & 16 & 1057 & $1 \%$ \\
\hline Set $5 \mathrm{k} 8 \mathrm{~J} 48$ & 120 & 953 & $11 \%$ \\
\hline Set $5 \mathrm{k} 8 \mathrm{LWL}$ & 68 & 1005 & $6 \%$ \\
\hline Set 5 k11 Hyperpipes & 103 & 970 & $10 \%$ \\
\hline Set 5 k11 Naive Bayes & 117 & 956 & $11 \%$ \\
\hline Set $5 \mathrm{k} 11$ OneR & 18 & 1055 & $2 \%$ \\
\hline Set $5 \mathrm{k} 11 \mathrm{~J} 48$ & 76 & 997 & $7 \%$ \\
\hline Set $5 \mathrm{k} 11 \mathrm{LWL}$ & 74 & 999 & $7 \%$ \\
\hline Set 5 k14 Hyperpipes & 130 & 943 & $12 \%$ \\
\hline Set 5 k14 Naive Bayes & 160 & 913 & $15 \%$ \\
\hline Set 5 k14 OneR & 17 & 1056 & $2 \%$ \\
\hline Set $5 \mathrm{k} 14 \mathrm{~J} 48$ & 101 & 972 & $9 \%$ \\
\hline Set $5 \mathrm{k} 14 \mathrm{LWL}$ & 65 & 1008 & $6 \%$ \\
\hline Set 6 k1 Hyperpipes & 181 & 860 & $17 \%$ \\
\hline Set 6 k1 Naive Bayes & 66 & 975 & $6 \%$ \\
\hline Set $6 \mathrm{k} 1$ OneR & 0 & 1041 & $0 \%$ \\
\hline Set $6 \mathrm{k} 1 \mathrm{~J} 48$ & 62 & 979 & $6 \%$ \\
\hline Set 6 k1 LWL & 68 & 973 & $7 \%$ \\
\hline Set 6 k3 Hyperpipes & 316 & 725 & $30 \%$ \\
\hline Set 6 k3 Naive Bayes & 294 & 747 & $28 \%$ \\
\hline Set $6 \mathrm{k} 3$ OneR & 0 & 1041 & $0 \%$ \\
\hline
\end{tabular}




\begin{tabular}{|c|c|c|c|}
\hline & Successes & Failures & Average of Success Rates \\
\hline Set $6 \mathrm{k} 3 \mathrm{~J} 48$ & 322 & 719 & $31 \%$ \\
\hline Set $6 \mathrm{k} 3 \mathrm{LWL}$ & 198 & 843 & $19 \%$ \\
\hline Set 6 k6 Hyperpipes & 577 & 464 & $55 \%$ \\
\hline Set 6 k6 Naive Bayes & 539 & 502 & $52 \%$ \\
\hline Set 6 k6 OneR & 0 & 1041 & $0 \%$ \\
\hline Set 6 k6 J48 & 312 & 729 & $30 \%$ \\
\hline Set 6 k6 LWL & 203 & 838 & $20 \%$ \\
\hline Set 6 k8 Hyperpipes & 514 & 527 & $49 \%$ \\
\hline Set 6 k8 Naive Bayes & 525 & 516 & $50 \%$ \\
\hline Set $6 \mathrm{k} 8$ OneR & 0 & 1041 & $0 \%$ \\
\hline Set $6 \mathrm{k} 8 \mathrm{~J} 48$ & 317 & 724 & $30 \%$ \\
\hline Set 6 k8 LWL & 196 & 845 & $19 \%$ \\
\hline Set 6 k11 Hyperpipes & 206 & 835 & $20 \%$ \\
\hline Set 6 k11 Naive Bayes & 504 & 537 & $48 \%$ \\
\hline Set 6 k11 OneR & 0 & 1041 & $0 \%$ \\
\hline Set $6 \mathrm{k} 11 \mathrm{~J} 48$ & 321 & 720 & $31 \%$ \\
\hline Set 6 k11 LWL & 174 & 867 & $17 \%$ \\
\hline Set 6 k14 Hyperpipes & 415 & 626 & $40 \%$ \\
\hline Set 6 k14 Naive Bayes & 475 & 566 & $46 \%$ \\
\hline Set 6 k14 OneR & 0 & 1041 & $0 \%$ \\
\hline Set 6 k14 J48 & 315 & 726 & $30 \%$ \\
\hline Set 6 k14 LWL & 146 & 895 & $14 \%$ \\
\hline
\end{tabular}




\begin{tabular}{|c|c|c|c|} 
& Successes & Failures & Average of Success Rates \\
\hline Set 7 k1 Hyperpipes & 362 & 611 & $37 \%$ \\
\hline Set 7 k1 Naive Bayes & 635 & 338 & $65 \%$ \\
\hline Set 7 k1 OneR & 596 & 377 & $61 \%$ \\
\hline Set 7 k1 J48 & 637 & 336 & $65 \%$ \\
\hline Set 7 k1 LWL & 644 & 329 & $66 \%$ \\
\hline Set 7 k3 Hyperpipes & 313 & 660 & $32 \%$ \\
\hline Set 7 k3 Naive Bayes & 601 & 372 & $62 \%$ \\
\hline Set 7 k3 OneR & 546 & 427 & $56 \%$ \\
\hline Set 7 k3 J48 & 624 & 349 & $64 \%$ \\
\hline Set 7 k3 LWL & 580 & 393 & $60 \%$ \\
\hline Set 7 k6 Hyperpipes & 128 & 845 & $13 \%$ \\
\hline Set 7 k6 Naive Bayes & 649 & 324 & $67 \%$ \\
\hline Set 7 k6 OneR & 589 & 384 & $61 \%$ \\
\hline Set 7 k6 J48 & 663 & 310 & $68 \%$ \\
\hline Set 7 k6 LWL & 605 & 368 & $62 \%$ \\
\hline Set 7 k8 Hyperpipes & 128 & 845 & $13 \%$ \\
\hline Set 7 k8 Naive Bayes & 557 & 416 & $57 \%$ \\
\hline Set 7 k8 OneR & 548 & 425 & $56 \%$ \\
\hline Set 7 k8 J48 & 601 & 372 & $62 \%$ \\
\hline Set 7 k8 LWL & 565 & 408 & $58 \%$ \\
\hline Set 7 k11 Hyperpipes & 120 & 853 & \\
\hline Set 7 k11 Naive Bayes & 502 & 471 & \\
\hline Continuing next page & & & $62 \%$ \\
\hline
\end{tabular}




\begin{tabular}{|c|c|c|c|} 
& Successes & Failures & Average of Success Rates \\
\hline Set 7 k11 OneR & 531 & 442 & $55 \%$ \\
\hline Set 7 k11 J48 & 566 & 407 & $58 \%$ \\
\hline Set 7 k11 LWL & 548 & 425 & $56 \%$ \\
\hline Set 7 k14 Hyperpipes & 137 & 836 & $14 \%$ \\
\hline Set 7 k14 Naive Bayes & 485 & 488 & $50 \%$ \\
\hline Set 7 k14 OneR & 516 & 457 & $53 \%$ \\
\hline Set 7 k14 J48 & 542 & 431 & $56 \%$ \\
\hline Set 7 k14 LWL & 537 & 436 & $55 \%$ \\
\hline Set 8 k1 Hyperpipes & 322 & 831 & $28 \%$ \\
\hline Set 8 k1 Naive Bayes & 666 & 487 & $58 \%$ \\
\hline Set 8 k1 OneR & 126 & 1027 & $11 \%$ \\
\hline Set 8 k1 J48 & 593 & 560 & $51 \%$ \\
\hline Set 8 k1 LWL & 612 & 541 & $53 \%$ \\
\hline Set 8 k3 Hyperpipes & 356 & 797 & $31 \%$ \\
\hline Set 8 k3 Naive Bayes & 534 & 619 & $46 \%$ \\
\hline Set 8 k3 OneR & 126 & 1027 & $11 \%$ \\
\hline Set 8 k3 J48 & 601 & 552 & $52 \%$ \\
\hline Set 8 k3 LWL & 375 & 778 & $33 \%$ \\
\hline Set 8 k6 Hyperpipes & 434 & 719 & $38 \%$ \\
\hline Set 8 k6 Naive Bayes & 659 & 494 & $11 \%$ \\
\hline Set 8 k6 OneR & 126 & 1027 & $60 \%$ \\
\hline Set 8 k6 J48 & 689 & 464 & \\
\hline Continuing next page & & & $57 \%$ \\
\hline
\end{tabular}




\begin{tabular}{|c|c|c|c|} 
& Successes & Failures & Average of Success Rates \\
\hline Set 8 k6 LWL & 475 & 678 & $41 \%$ \\
\hline Set 8 k8 Hyperpipes & 402 & 751 & $35 \%$ \\
\hline Set 8 k8 Naive Bayes & 643 & 510 & $56 \%$ \\
\hline Set 8 k8 OneR & 125 & 1028 & $11 \%$ \\
\hline Set 8 k8 J48 & 657 & 496 & $57 \%$ \\
\hline Set 8 k8 LWL & 457 & 696 & $40 \%$ \\
\hline Set 8 k11 Hyperpipes & 280 & 873 & $24 \%$ \\
\hline Set 8 k11 Naive Bayes & 458 & 695 & $40 \%$ \\
\hline Set 8 k11 OneR & 124 & 1029 & $11 \%$ \\
\hline Set 8 k11 J48 & 522 & 631 & $45 \%$ \\
\hline Set 8 k11 LWL & 337 & 816 & $29 \%$ \\
\hline Set 8 k14 Hyperpipes & 238 & 915 & $21 \%$ \\
\hline Set 8 k14 Naive Bayes & 558 & 595 & $48 \%$ \\
\hline Set 8 k14 OneR & 121 & 1032 & $10 \%$ \\
\hline Set 8 k14 J48 & 584 & 569 & $51 \%$ \\
\hline Set 8 k14 LWL & 293 & 860 & $25 \%$ \\
\hline Set 9 k1 Hyperpipes & 280 & 766 & $27 \%$ \\
\hline Set 9 k1 Naive Bayes & 776 & 270 & $74 \%$ \\
\hline Set 9 k1 OneR & 354 & 692 & $29 \%$ \\
\hline Set 9 k1 J48 & 772 & 274 & \\
\hline Set 9 k1 LWL & 786 & 260 & $73 \%$ \\
\hline Set 9 k3 Hyperpipes & 307 & 739 & \\
\hline Continuing next page & & & $54 \%$ \\
\hline
\end{tabular}




\begin{tabular}{|c|c|c|c|} 
& Successes & Failures & Average of Success Rates \\
\hline Set 9 k3 Naive Bayes & 750 & 296 & $72 \%$ \\
\hline Set 9 k3 OneR & 349 & 697 & $33 \%$ \\
\hline Set 9 k3 J48 & 742 & 304 & $71 \%$ \\
\hline Set 9 k3 LWL & 632 & 414 & $60 \%$ \\
\hline Set 9 k6 Hyperpipes & 264 & 782 & $25 \%$ \\
\hline Set 9 k6 Naive Bayes & 721 & 325 & $69 \%$ \\
\hline Set 9 k6 OneR & 350 & 696 & $33 \%$ \\
\hline Set 9 k6 J48 & 728 & 318 & $70 \%$ \\
\hline Set 9 k6 LWL & 520 & 526 & $50 \%$ \\
\hline Set 9 k8 Hyperpipes & 263 & 783 & $25 \%$ \\
\hline Set 9 k8 Naive Bayes & 700 & 346 & $67 \%$ \\
\hline Set 9 k8 OneR & 350 & 696 & $33 \%$ \\
\hline Set 9 k8 J48 & 746 & 300 & $71 \%$ \\
\hline Set 9 k8 LWL & 490 & 556 & $47 \%$ \\
\hline Set 9 k11 Hyperpipes & 266 & 780 & $25 \%$ \\
\hline Set 9 k11 Naive Bayes & 692 & 354 & $66 \%$ \\
\hline Set 9 k11 OneR & 349 & 697 & $33 \%$ \\
\hline Set 9 k11 J48 & 763 & 283 & $73 \%$ \\
\hline Set 9 k11 LWL & 480 & 566 & $83 \%$ \\
\hline Set 9 k14 Hyperpipes & 216 & 830 & \\
\hline Set 9 k14 Naive Bayes & 645 & 401 & 697 \\
\hline Set 9 k14 OneR & 349 & 697 & \\
\hline Continuing next page & & & $63 \%$ \\
\hline
\end{tabular}




\begin{tabular}{|c|c|c|c|} 
& Successes & Failures & Average of Success Rates \\
\hline Set 9 k14 J48 & 718 & 328 & $69 \%$ \\
\hline Set 9 k14 LWL & 493 & 553 & $47 \%$ \\
\hline Set 10 k1 Hyperpipes & 687 & 410 & $63 \%$ \\
\hline Set 10 k1 Naive Bayes & 841 & 256 & $77 \%$ \\
\hline Set 10 k1 OneR & 161 & 936 & $15 \%$ \\
\hline Set 10 k1 J48 & 849 & 248 & $77 \%$ \\
\hline Set 10 k1 LWL & 851 & 246 & $78 \%$ \\
\hline Set 10 k3 Hyperpipes & 636 & 461 & $58 \%$ \\
\hline Set 10 k3 Naive Bayes & 867 & 230 & $79 \%$ \\
\hline Set 10 k3 OneR & 157 & 940 & $14 \%$ \\
\hline Set 10 k3 J48 & 873 & 224 & $80 \%$ \\
\hline Set 10 k3 LWL & 739 & 358 & $67 \%$ \\
\hline Set 10 k6 Hyperpipes & 623 & 474 & $57 \%$ \\
\hline Set 10 k6 Naive Bayes & 851 & 246 & $78 \%$ \\
\hline Set 10 k6 OneR & 153 & 944 & $14 \%$ \\
\hline Set 10 k6 J48 & 869 & 228 & $79 \%$ \\
\hline Set 10 k6 LWL & 665 & 432 & $61 \%$ \\
\hline Set 10 k8 Hyperpipes & 626 & 471 & $57 \%$ \\
\hline Set 10 k8 Naive Bayes & 814 & 283 & $79 \%$ \\
\hline Set 10 k8 OneR & 151 & 946 & $59 \%$ \\
\hline Set 10 k8 J48 & 862 & 235 & $49 \%$ \\
\hline Set 10 k8 LWL & 647 & 450 & \\
\hline Continuing next page & & & \\
\hline
\end{tabular}




\begin{tabular}{|c|c|c|c|} 
& Successes & Failures & Average of Success Rates \\
\hline Set 10 k11 Hyperpipes & 619 & 478 & $56 \%$ \\
\hline Set 10 k11 Naive Bayes & 802 & 295 & $73 \%$ \\
\hline Set 10 k11 OneR & 151 & 946 & $14 \%$ \\
\hline Set 10 k11 J48 & 842 & 255 & $77 \%$ \\
\hline Set 10 k11 LWL & 618 & 479 & $56 \%$ \\
\hline Set 10 k14 Hyperpipes & 255 & 842 & $23 \%$ \\
\hline Set 10 k14 Naive Bayes & 482 & 615 & $44 \%$ \\
\hline Set 10 k14 OneR & 163 & 934 & $15 \%$ \\
\hline Set 10 k14 J48 & 605 & 492 & $55 \%$ \\
\hline Set 10 k14 LWL & 370 & 727 & $34 \%$ \\
\hline
\end{tabular}

Table B.3: Filtering success rates by all factors for all datasets

\begin{tabular}{|c|c|c|c|} 
& Successes & Failures & Average of Success Rates \\
\hline Set 1 k1 Hyperpipes & 453 & 114 & $80 \%$ \\
\hline Set 1 k1 Naive Bayes & 393 & 174 & $69 \%$ \\
\hline Set 1 k1 OneR & N/A & N/A & N/A \\
\hline Set 1 k1 J48 & 479 & 88 & $84 \%$ \\
\hline Set 1 k1 LWL & 463 & 104 & $82 \%$ \\
\hline Set 1 k3 Hyperpipes & 473 & 94 & $83 \%$ \\
\hline Set 1 k3 Naive Bayes & 487 & 80 & $86 \%$ \\
\hline Set 1 k3 OneR & N/A & N/A & N/A \\
\hline Set 1 k3 J48 & 491 & 76 & $87 \%$ \\
\hline $\begin{array}{c}\text { Set 1 k3 LWL } \\
\text { Continuing next page }\end{array}$ & 392 & 175 & $69 \%$ \\
\hline
\end{tabular}




\begin{tabular}{|c|c|c|c|} 
& Successes & Failures & Average of Success Rates \\
\hline Set 1 k6 Hyperpipes & 464 & 103 & $82 \%$ \\
\hline Set 1 k6 Naive Bayes & 490 & 77 & $86 \%$ \\
\hline Set 1 k6 OneR & N/A & N/A & N/A \\
\hline Set 1 k6 J48 & 530 & 37 & $93 \%$ \\
\hline Set 1 k6 LWL & 385 & 182 & $68 \%$ \\
\hline Set 1 k8 Hyperpipes & 459 & 108 & $81 \%$ \\
\hline Set 1 k8 Naive Bayes & 475 & 92 & $84 \%$ \\
\hline Set 1 k8 OneR & N/A & N/A & N/A \\
\hline Set 1 k8 J48 & 537 & 30 & $95 \%$ \\
\hline Set 1 k8 LWL & 374 & 193 & $66 \%$ \\
\hline Set 1 k11 Hyperpipes & 448 & 119 & $79 \%$ \\
\hline Set 1 k11 Naive Bayes & 451 & 116 & $80 \%$ \\
\hline Set 1 k11 OneR & N/A & N/A & N/A \\
\hline Set 1 k11 J48 & 567 & 0 & $100 \%$ \\
\hline Set 1 k11 LWL & 361 & 206 & $64 \%$ \\
\hline Set 1 k14 Hyperpipes & 435 & 132 & $77 \%$ \\
\hline Set 1 k14 Naive Bayes & 432 & 135 & $76 \%$ \\
\hline Set 1 k14 OneR & N/A & N/A & $100 \%$ \\
\hline Set 1 k14 J48 & 565 & 2 & $206 \%$ \\
\hline Set 1 k14 LWL & 361 & 206 & \\
\hline Set 2 k1 Hyperpipes & 183 & 0 & $00 \%$ \\
\hline Set 2 k1 Naive Bayes & 193 & 0 & \\
\hline Continuing next page & & & \\
\hline
\end{tabular}




\begin{tabular}{|c|c|c|c|} 
& Successes & Failures & Average of Success Rates \\
\hline Set 2 k1 OneR & N/A & N/A & N/A \\
\hline Set 2 k1 J48 & 193 & 0 & $100 \%$ \\
\hline Set 2 k1 LWL & 193 & 0 & $100 \%$ \\
\hline Set 2 k3 Hyperpipes & 183 & 0 & $100 \%$ \\
\hline Set 2 k3 Naive Bayes & 193 & 0 & $100 \%$ \\
\hline Set 2 k3 OneR & N/A & N/A & N/A \\
\hline Set 2 k3 J48 & 193 & 0 & $100 \%$ \\
\hline Set 2 k3 LWL & 193 & 0 & $100 \%$ \\
\hline Set 2 k6 Hyperpipes & 183 & 0 & $100 \%$ \\
\hline Set 2 k6 Naive Bayes & 193 & 0 & $100 \%$ \\
\hline Set 2 k6 OneR & N/A & N/A & N/A \\
\hline Set 2 k6 J48 & 193 & 0 & $100 \%$ \\
\hline Set 2 k6 LWL & 193 & 0 & $100 \%$ \\
\hline Set 2 k8 Hyperpipes & 183 & 0 & $100 \%$ \\
\hline Set 2 k8 Naive Bayes & 193 & 0 & $100 \%$ \\
\hline Set 2 k8 OneR & N/A & N/A & N/A \\
\hline Set 2 k8 J48 & 193 & 0 & $100 \%$ \\
\hline Set 2 k8 LWL & 193 & 0 & $100 \%$ \\
\hline Set 2 k11 Hyperpipes & 183 & 0 & $100 \%$ \\
\hline Set 2 k11 Naive Bayes & 193 & 0 & N/A \\
\hline Set 2 k11 OneR & N/A & N/A & $100 \%$ \\
\hline Set 2 k11 J48 & 193 & 0 & $00 \%$ \\
Continuing next page & & & 0 \\
\hline
\end{tabular}




\begin{tabular}{|c|c|c|c|}
\hline & Successes & Failures & Average of Success Rates \\
\hline Set $2 \mathrm{k} 11 \mathrm{LWL}$ & 193 & 0 & $100 \%$ \\
\hline Set 2 k14 Hyperpipes & 183 & 0 & $100 \%$ \\
\hline Set 2 k14 Naive Bayes & 193 & 0 & $100 \%$ \\
\hline Set 2 k14 OneR & N/A & N/A & N/A \\
\hline Set $2 \mathrm{k} 14 \mathrm{~J} 48$ & 193 & 0 & $100 \%$ \\
\hline Set 2 k14 LWL & 193 & 0 & $100 \%$ \\
\hline Set 3 k1 Hyperpipes & 115 & 9 & $93 \%$ \\
\hline Set 3 k1 Naive Bayes & 108 & 16 & $87 \%$ \\
\hline Set $3 \mathrm{k} 1$ OneR & N/A & N/A & N/A \\
\hline Set $3 \mathrm{k} 1 \mathrm{~J} 48$ & 124 & 0 & $100 \%$ \\
\hline Set $3 \mathrm{k} 1 \mathrm{LWL}$ & 116 & 8 & $94 \%$ \\
\hline Set 3 k3 Hyperpipes & 118 & 6 & $95 \%$ \\
\hline Set 3 k3 Naive Bayes & 116 & 8 & $94 \%$ \\
\hline Set $3 \mathrm{k} 3$ OneR & N/A & N/A & N/A \\
\hline Set $3 \mathrm{k} 3 \mathrm{~J} 48$ & 124 & 0 & $100 \%$ \\
\hline Set 3 k3 LWL & 116 & 8 & $94 \%$ \\
\hline Set 3 k6 Hyperpipes & 118 & 6 & $95 \%$ \\
\hline Set 3 k6 Naive Bayes & 117 & 7 & $94 \%$ \\
\hline Set 3 k6 OneR & N/A & N/A & N/A \\
\hline Set $3 \mathrm{k} 6 \mathrm{~J} 48$ & 123 & 1 & $99 \%$ \\
\hline Set $3 \mathrm{k} 6 \mathrm{LWL}$ & 114 & 10 & $92 \%$ \\
\hline Set 3 k8 Hyperpipes & 119 & 5 & $96 \%$ \\
\hline
\end{tabular}




\begin{tabular}{|c|c|c|c|} 
& Successes & Failures & Average of Success Rates \\
\hline Set 3 k8 Naive Bayes & 117 & 7 & $94 \%$ \\
\hline Set 3 k8 OneR & N/A & N/A & N/A \\
\hline Set 3 k8 J48 & 124 & 0 & $100 \%$ \\
\hline Set 3 k8 LWL & 112 & 12 & $90 \%$ \\
\hline Set 3 k11 Hyperpipes & 117 & 7 & $94 \%$ \\
\hline Set 3 k11 Naive Bayes & 115 & 9 & $93 \%$ \\
\hline Set 3 k11 OneR & N/A & N/A & N/A \\
\hline Set 3 k11 J48 & 124 & 0 & $100 \%$ \\
\hline Set 3 k11 LWL & 113 & 11 & $91 \%$ \\
\hline Set 3 k14 Hyperpipes & 118 & 6 & $95 \%$ \\
\hline Set 3 k14 Naive Bayes & 115 & 9 & $93 \%$ \\
\hline Set 3 k14 OneR & N/A & N/A & N/A \\
\hline Set 3 k14 J48 & 123 & 1 & $99 \%$ \\
\hline Set 3 k14 LWL & 114 & 10 & $92 \%$ \\
\hline Set 4 k1 Hyperpipes & 490 & 38 & $93 \%$ \\
\hline Set 4 k1 Naive Bayes & 479 & 49 & $91 \%$ \\
\hline Set 4 k1 OneR & N/A & N/A & $96 \%$ \\
\hline Set 4 k1 J48 & 507 & 21 & $39 \%$ \\
\hline Set 4 k1 LWL & 489 & 39 & \\
\hline Set 4 k3 Hyperpipes & 497 & 31 & 30 \\
\hline Set 4 k3 Naive Bayes & 498 & 30 & N/A \\
\hline Set 4 k3 OneR & N/A & & $94 \%$ \\
Continuing next page & & & $96 \%$ \\
\hline
\end{tabular}




\begin{tabular}{|c|c|c|c|} 
& Successes & Failures & Average of Success Rates \\
\hline Set 4 k3 J48 & 528 & 0 & $100 \%$ \\
\hline Set 4 k3 LWL & 469 & 59 & $89 \%$ \\
\hline Set 4 k6 Hyperpipes & 498 & 30 & $94 \%$ \\
\hline Set 4 k6 Naive Bayes & 506 & 22 & $96 \%$ \\
\hline Set 4 k6 OneR & N/A & N/A & N/A \\
\hline Set 4 k6 J48 & 528 & 0 & $100 \%$ \\
\hline Set 4 k6 LWL & 458 & 70 & $87 \%$ \\
\hline Set 4 k8 Hyperpipes & 498 & 30 & $94 \%$ \\
\hline Set 4 k8 Naive Bayes & 509 & 19 & $96 \%$ \\
\hline Set 4 k8 OneR & N/A & N/A & N/A \\
\hline Set 4 k8 J48 & 528 & 0 & $100 \%$ \\
\hline Set 4 k8 LWL & 459 & 69 & $87 \%$ \\
\hline Set 4 k11 Hyperpipes & 493 & 35 & $93 \%$ \\
\hline Set 4 k11 Naive Bayes & 499 & 29 & $95 \%$ \\
\hline Set 4 k11 OneR & N/A & N/A & N/A \\
\hline Set 4 k11 J48 & 528 & 0 & $100 \%$ \\
\hline Set 4 k11 LWL & 464 & 64 & $88 \%$ \\
\hline Set 4 k14 Hyperpipes & 467 & 61 & $88 \%$ \\
\hline Set 4 k14 Naive Bayes & 398 & 130 & $75 \%$ \\
\hline Set 4 k14 OneR & N/A & N/A & 74 \\
\hline Set 4 k14 J48 & 454 & 74 & 177 \\
\hline Set 4 k14 LWL & 351 & & \\
\hline Continuing next page & & & No \\
\hline
\end{tabular}




\begin{tabular}{|c|c|c|c|} 
& Successes & Failures & Average of Success Rates \\
\hline Set 5 k1 Hyperpipes & 255 & 818 & $24 \%$ \\
\hline Set 5 k1 Naive Bayes & 337 & 736 & $31 \%$ \\
\hline Set 5 k1 OneR & N/A & N/A & N/A \\
\hline Set 5 k1 J48 & 287 & 786 & $27 \%$ \\
\hline Set 5 k1 LWL & 293 & 780 & $27 \%$ \\
\hline Set 5 k3 Hyperpipes & 321 & 752 & $30 \%$ \\
\hline Set 5 k3 Naive Bayes & 992 & 81 & $92 \%$ \\
\hline Set 5 k3 OneR & N/A & N/A & N/A \\
\hline Set 5 k3 J48 & 716 & 357 & $67 \%$ \\
\hline Set 5 k3 LWL & 291 & 782 & $27 \%$ \\
\hline Set 5 k6 Hyperpipes & 319 & 754 & $30 \%$ \\
\hline Set 5 k6 Naive Bayes & 987 & 86 & $92 \%$ \\
\hline Set 5 k6 OneR & N/A & N/A & N/A \\
\hline Set 5 k6 J48 & 558 & 515 & $52 \%$ \\
\hline Set 5 k6 LWL & 244 & 829 & $23 \%$ \\
\hline Set 5 k8 Hyperpipes & 314 & 759 & $29 \%$ \\
\hline Set 5 k8 Naive Bayes & 967 & 106 & $90 \%$ \\
\hline Set 5 k8 OneR & N/A & N/A & $39 \%$ \\
\hline Set 5 k8 J48 & 417 & 656 & 803 \\
\hline Set 5 k8 LWL & 270 & 772 & $28 \%$ \\
\hline Set 5 k11 Hyperpipes & 301 & 201 & \\
\hline Set 5 k11 Naive Bayes & 872 & & \\
\hline Continuing next page & & & $29 \%$ \\
\hline
\end{tabular}




\begin{tabular}{|c|c|c|c|} 
& Successes & Failures & Average of Success Rates \\
\hline Set 5 k11 OneR & N/A & N/A & N/A \\
\hline Set 5 k11 J48 & 1023 & 50 & $95 \%$ \\
\hline Set 5 k11 LWL & 264 & 809 & $25 \%$ \\
\hline Set 5 k14 Hyperpipes & 279 & 794 & $26 \%$ \\
\hline Set 5 k14 Naive Bayes & 289 & 784 & $27 \%$ \\
\hline Set 5 k14 OneR & N/A & N/A & N/A \\
\hline Set 5 k14 J48 & 1032 & 41 & $96 \%$ \\
\hline Set 5 k14 LWL & 232 & 841 & $22 \%$ \\
\hline Set 6 k1 Hyperpipes & 400 & 641 & $38 \%$ \\
\hline Set 6 k1 Naive Bayes & 382 & 659 & $37 \%$ \\
\hline Set 6 k1 OneR & N/A & N/A & N/A \\
\hline Set 6 k1 J48 & 539 & 502 & $52 \%$ \\
\hline Set 6 k1 LWL & 507 & 534 & $49 \%$ \\
\hline Set 6 k3 Hyperpipes & 661 & 380 & $63 \%$ \\
\hline Set 6 k3 Naive Bayes & 706 & 335 & $68 \%$ \\
\hline Set 6 k3 OneR & N/A & N/A & N/A \\
\hline Set 6 k3 J48 & 713 & 328 & $68 \%$ \\
\hline Set 6 k3 LWL & 289 & 752 & $28 \%$ \\
\hline Set 6 k6 Hyperpipes & 658 & 383 & 102 \\
\hline Set 6 k6 Naive Bayes & 939 & 102 & \\
\hline Set 6 k6 OneR & N/A & N/A & \\
\hline Set 6 k6 J48 & 744 & 297 & \\
\hline Continuing next page & & & N/A \\
\hline
\end{tabular}




\begin{tabular}{|c|c|c|c|} 
& Successes & Failures & Average of Success Rates \\
\hline Set 6 k6 LWL & 300 & 741 & $29 \%$ \\
\hline Set 6 k8 Hyperpipes & 657 & 384 & $63 \%$ \\
\hline Set 6 k8 Naive Bayes & 922 & 119 & $89 \%$ \\
\hline Set 6 k8 OneR & N/A & N/A & N/A \\
\hline Set 6 k8 J48 & 789 & 252 & $76 \%$ \\
\hline Set 6 k8 LWL & 304 & 737 & $29 \%$ \\
\hline Set 6 k11 Hyperpipes & 655 & 386 & $63 \%$ \\
\hline Set 6 k11 Naive Bayes & 893 & 148 & $86 \%$ \\
\hline Set 6 k11 OneR & N/A & N/A & N/A \\
\hline Set 6 k11 J48 & 832 & 209 & $80 \%$ \\
\hline Set 6 k11 LWL & 296 & 745 & $28 \%$ \\
\hline Set 6 k14 Hyperpipes & 645 & 396 & $62 \%$ \\
\hline Set 6 k14 Naive Bayes & 862 & 179 & $83 \%$ \\
\hline Set 6 k14 OneR & N/A & N/A & N/A \\
\hline Set 6 k14 J48 & 926 & 115 & $89 \%$ \\
\hline Set 6 k14 LWL & 278 & 763 & $27 \%$ \\
\hline Set 7 k1 Hyperpipes & 966 & 7 & $99 \%$ \\
\hline Set 7 k1 Naive Bayes & 965 & 8 & $99 \%$ \\
\hline Set 7 k1 OneR & N/A & N/A & $99 \%$ \\
\hline Set 7 k1 J48 & 970 & 3 & $600 \%$ \\
\hline Set 7 k1 LWL & 967 & 6 & 6 \\
\hline Set 7 k3 Hyperpipes & 967 & 6 & \\
\hline Continuing next page & & & N/A \\
\hline
\end{tabular}




\begin{tabular}{|c|c|c|c|} 
& Successes & Failures & Average of Success Rates \\
\hline Set 7 k3 Naive Bayes & 969 & 4 & $100 \%$ \\
\hline Set 7 k3 OneR & N/A & N/A & N/A \\
\hline Set 7 k3 J48 & 967 & 6 & $99 \%$ \\
\hline Set 7 k3 LWL & 958 & 15 & $98 \%$ \\
\hline Set 7 k6 Hyperpipes & 967 & 6 & $99 \%$ \\
\hline Set 7 k6 Naive Bayes & 968 & 5 & $99 \%$ \\
\hline Set 7 k6 OneR & N/A & N/A & N/A \\
\hline Set 7 k6 J48 & 971 & 2 & $100 \%$ \\
\hline Set 7 k6 LWL & 966 & 7 & $99 \%$ \\
\hline Set 7 k8 Hyperpipes & 967 & 6 & $99 \%$ \\
\hline Set 7 k8 Naive Bayes & 967 & 6 & $99 \%$ \\
\hline Set 7 k8 OneR & N/A & N/A & N/A \\
\hline Set 7 k8 J48 & 966 & 7 & $99 \%$ \\
\hline Set 7 k8 LWL & 956 & 17 & $98 \%$ \\
\hline Set 7 k11 Hyperpipes & 967 & 6 & $99 \%$ \\
\hline Set 7 k11 Naive Bayes & 968 & 5 & $99 \%$ \\
\hline Set 7 k11 OneR & N/A & N/A & $99 \%$ \\
\hline Set 7 k11 J48 & 965 & 8 & 12 \\
\hline Set 7 k11 LWL & 961 & 6 & N/A \\
\hline Set 7 k14 Hyperpipes & 967 & 5 & N/A \\
\hline Set 7 k14 Naive Bayes & 968 & & $99 \%$ \\
\hline Set 7 k14 OneR & N/A & $69 \%$ \\
Continuing next page & & $69 \%$ \\
\hline
\end{tabular}




\begin{tabular}{|c|c|c|c|} 
& Successes & Failures & Average of Success Rates \\
\hline Set 7 k14 J48 & 962 & 11 & $99 \%$ \\
\hline Set 7 k14 LWL & 959 & 14 & $99 \%$ \\
\hline Set 8 k1 Hyperpipes & 1046 & 107 & $91 \%$ \\
\hline Set 8 k1 Naive Bayes & 992 & 161 & $86 \%$ \\
\hline Set 8 k1 OneR & N/A & N/A & N/A \\
\hline Set 8 k1 J48 & 1065 & 88 & $92 \%$ \\
\hline Set 8 k1 LWL & 1053 & 100 & $91 \%$ \\
\hline Set 8 k3 Hyperpipes & 1083 & 70 & $94 \%$ \\
\hline Set 8 k3 Naive Bayes & 1083 & 70 & $94 \%$ \\
\hline Set 8 k3 OneR & N/A & N/A & N/A \\
\hline Set 8 k3 J48 & 1079 & 74 & $94 \%$ \\
\hline Set 8 k3 LWL & 895 & 258 & $78 \%$ \\
\hline Set 8 k6 Hyperpipes & 1079 & 74 & $94 \%$ \\
\hline Set 8 k6 Naive Bayes & 1083 & 70 & $94 \%$ \\
\hline Set 8 k6 OneR & N/A & N/A & N/A \\
\hline Set 8 k6 J48 & 1105 & 48 & $96 \%$ \\
\hline Set 8 k6 LWL & 864 & 289 & $75 \%$ \\
\hline Set 8 k8 Hyperpipes & 1067 & 86 & $93 \%$ \\
\hline Set 8 k8 Naive Bayes & 1065 & 88 & $97 \%$ \\
\hline Set 8 k8 OneR & N/A & N/A & \\
\hline Set 8 k8 J48 & 1117 & 36 & $34 \%$ \\
\hline Set 8 k8 LWL & 853 & 300 & \\
\hline Continuing next page & & & \\
\hline
\end{tabular}




\begin{tabular}{|c|c|c|c|}
\hline & Successes & Failures & Average of Success Rates \\
\hline Set 8 k11 Hyperpipes & 1052 & 101 & $91 \%$ \\
\hline Set 8 k11 Naive Bayes & 1046 & 107 & $91 \%$ \\
\hline Set 8 k11 OneR & N/A & N/A & N/A \\
\hline Set 8 k11 J48 & 1153 & 0 & $100 \%$ \\
\hline Set 8 k11 LWL & 833 & 320 & $72 \%$ \\
\hline Set 8 k14 Hyperpipes & 1035 & 118 & $90 \%$ \\
\hline Set 8 k14 Naive Bayes & 1008 & 145 & $87 \%$ \\
\hline Set 8 k14 OneR & N/A & N/A & N/A \\
\hline Set 8 k14 J48 & 1152 & 1 & $100 \%$ \\
\hline Set 8 k14 LWL & 793 & 360 & $69 \%$ \\
\hline Set 9 k1 Hyperpipes & 1029 & 17 & $98 \%$ \\
\hline Set 9 k1 Naive Bayes & 1017 & 29 & $97 \%$ \\
\hline Set $9 \mathrm{k} 1$ OneR & N/A & N/A & N/A \\
\hline Set $9 \mathrm{k} 1 \mathrm{~J} 48$ & 1036 & 10 & $99 \%$ \\
\hline Set $9 \mathrm{k} 1 \mathrm{LWL}$ & 1026 & 20 & $98 \%$ \\
\hline Set 9 k3 Hyperpipes & 1029 & 17 & $98 \%$ \\
\hline Set 9 k3 Naive Bayes & 1022 & 24 & $98 \%$ \\
\hline Set $9 \mathrm{k} 3$ OneR & N/A & N/A & N/A \\
\hline Set $9 \mathrm{k} 3 \mathrm{~J} 48$ & 1042 & 4 & $100 \%$ \\
\hline Set 9 k3 LWL & 957 & 89 & $91 \%$ \\
\hline Set 9 k6 Hyperpipes & 1029 & 17 & $98 \%$ \\
\hline Set 9 k6 Naive Bayes & 1028 & 18 & $98 \%$ \\
\hline Continuing next page & & & \\
\hline
\end{tabular}




\begin{tabular}{|c|c|c|c|} 
& Successes & Failures & Average of Success Rates \\
\hline Set 9 k6 OneR & N/A & N/A & N/A \\
\hline Set 9 k6 J48 & 1041 & 5 & $100 \%$ \\
\hline Set 9 k6 LWL & 971 & 75 & $93 \%$ \\
\hline Set 9 k8 Hyperpipes & 1029 & 17 & $98 \%$ \\
\hline Set 9 k8 Naive Bayes & 1021 & 25 & $98 \%$ \\
\hline Set 9 k8 OneR & N/A & N/A & N/A \\
\hline Set 9 k8 J48 & 1046 & 0 & $100 \%$ \\
\hline Set 9 k8 LWL & 956 & 90 & $91 \%$ \\
\hline Set 9 k11 Hyperpipes & 1028 & 18 & $98 \%$ \\
\hline Set 9 k11 Naive Bayes & 1009 & 37 & $96 \%$ \\
\hline Set 9 k11 OneR & N/A & N/A & N/A \\
\hline Set 9 k11 J48 & 1045 & 1 & $100 \%$ \\
\hline Set 9 k11 LWL & 957 & 89 & $91 \%$ \\
\hline Set 9 k14 Hyperpipes & 1027 & 19 & $98 \%$ \\
\hline Set 9 k14 Naive Bayes & 1002 & 44 & $96 \%$ \\
\hline Set 9 k14 OneR & N/A & N/A & N/A \\
\hline Set 9 k14 J48 & 1044 & 2 & $100 \%$ \\
\hline Set 9 k14 LWL & 988 & 58 & $94 \%$ \\
\hline Set 10 k1 Hyperpipes & 1053 & 44 & $96 \%$ \\
\hline Set 10 k1 Naive Bayes & 1037 & 60 & N/A \\
\hline Set 10 k1 OneR & N/A & 25 & \\
\hline Set 10 k1 J48 & 1072 & & $95 \%$ \\
\hline Continuing next page & & & \\
\hline
\end{tabular}




\begin{tabular}{|c|c|c|c|} 
& Successes & Failures & Average of Success Rates \\
\hline Set 10 k1 LWL & 1051 & 46 & $96 \%$ \\
\hline Set 10 k3 Hyperpipes & 1062 & 35 & $97 \%$ \\
\hline Set 10 k3 Naive Bayes & 1056 & 41 & $96 \%$ \\
\hline Set 10 k3 OneR & N/A & N/A & N/A \\
\hline Set 10 k3 J48 & 1092 & 5 & $100 \%$ \\
\hline Set 10 k3 LWL & 993 & 104 & $91 \%$ \\
\hline Set 10 k6 Hyperpipes & 1064 & 33 & $97 \%$ \\
\hline Set 10 k6 Naive Bayes & 1066 & 31 & $97 \%$ \\
\hline Set 10 k6 OneR & N/A & N/A & N/A \\
\hline Set 10 k6 J48 & 1097 & 0 & $100 \%$ \\
\hline Set 10 k6 LWL & 967 & 130 & $88 \%$ \\
\hline Set 10 k8 Hyperpipes & 1064 & 33 & $97 \%$ \\
\hline Set 10 k8 Naive Bayes & 1067 & 30 & $97 \%$ \\
\hline Set 10 k8 OneR & N/A & N/A & N/A \\
\hline Set 10 k8 J48 & 1096 & 1 & $100 \%$ \\
\hline Set 10 k8 LWL & 975 & 122 & $89 \%$ \\
\hline Set 10 k11 Hyperpipes & 1059 & 38 & $97 \%$ \\
\hline Set 10 k11 Naive Bayes & 1055 & 42 & $96 \%$ \\
\hline Set 10 k11 OneR & N/A & N/A & $100 \%$ \\
\hline Set 10 k11 J48 & 1097 & 0 & 118 \\
\hline Set 10 k11 LWL & 979 & 74 & \\
\hline Set 10 k14 Hyperpipes & 1023 & & No \\
\hline Continuing next page & & & \\
\hline
\end{tabular}




\begin{tabular}{|c|c|c|c|} 
& Successes & Failures & Average of Success Rates \\
\hline Set 10 k14 Naive Bayes & 868 & 229 & $79 \%$ \\
\hline Set 10 k14 OneR & N/A & N/A & N/A \\
\hline Set 10 k14 J48 & 941 & 156 & $86 \%$ \\
\hline Set 10 k14 LWL & 771 & 326 & $70 \%$ \\
\hline
\end{tabular}

Table B.4: List of document file extensions allowed through preprocessing whitelist

\begin{tabular}{|c|c|c|c|c|c|c|}
\hline \$p & 0 & 0 & 1 & 1 sp & 2 & 2 \\
\hline 212 & 3 & 3 & 301 & $3 \mathrm{~d}$ & $3 \mathrm{~d}$ & $3 \mathrm{~d} 6$ \\
\hline $3 \mathrm{df}$ & $3 \mathrm{dg}$ & $3 \mathrm{dz}$ & 4 & $4 \mathrm{w} 7$ & $4 \mathrm{wt}$ & 602 \\
\hline a5r & a5w & a7p & a7r & aa & aad & ab65 \\
\hline abicollab & abs & abw & aca & acc & accdp & acp \\
\hline acp & acp & acrypt & ada & adb & adc & ade \\
\hline adn & ados & adp & adt & adv & adx & aep \\
\hline aepx & af2 & af3 & afd & aff & afp & aft \\
\hline agldei & aglsl & agp & agr & ahf & alb3 & alb4 \\
\hline alb5 & ald & ald5 & alg & ali & ali & all \\
\hline alt3 & alt5 & alt6 & amsm & amst & amx & and \\
\hline anl & ans & ans & ansr & anx & apa & apf \\
\hline apf & apo & applocalize & apr & apr & apt & apw \\
\hline asd & asn & asp & asp & asp & ast & asv \\
\hline at2 & at65 & ath & aup & av & aw & aw \\
\hline awp & aws & awt & aww & awwp & axg & axr \\
\hline awg & & & &
\end{tabular}

Continuing next page 


\begin{tabular}{|c|c|c|c|c|c|c|}
\hline b26 & b27 & $\mathrm{b} 4 \mathrm{~s}$ & $\mathrm{~b} 4 \mathrm{u}$ & bbl & bbprojectd & bc5 \\
\hline bcp & bdsproj & bdt2 & bdt3 & bean & bfx & bibtex \\
\hline bil & bina & biz & biz & bizdocument & bk & bkg \\
\hline $\mathrm{bkr}$ & bks & bld & blg & blg & blg & blt \\
\hline bmm & bobo & boc & bok & boo & book & book \\
\hline bookexport & ?booktemplate & brh & bro & bsb & btd & btf \\
\hline btw & btx & burn & burntheme & bwp & bxx & bzabw \\
\hline $\mathrm{c} 00$ & $\mathrm{c} 2 \mathrm{e}$ & cap & cap & cap & cap & cap \\
\hline cap & cap & cap & cap & cap & $\operatorname{cbf}$ & cbl \\
\hline $\operatorname{cbs}$ & $\mathrm{cbt}$ & $\mathrm{ccc}$ & $\operatorname{cch}$ & $\operatorname{cd} 2$ & $\mathrm{cdc}$ & $\mathrm{cdc}$ \\
\hline $\mathrm{cdc}$ & cdd & cdd & cdf & $\mathrm{cdk}$ & cdl & $\operatorname{cdmz}$ \\
\hline cdp & cds & $\mathrm{cdt}$ & cdt6 & $\operatorname{cdx}$ & $\mathrm{cdz}$ & cer \\
\hline cer & $\operatorname{cfd}$ & cfl & cfl & $\mathrm{cfm}$ & $\mathrm{cfr}$ & $\operatorname{cgdc}$ \\
\hline $\operatorname{ch} 4$ & che & chi & $\mathrm{chm}$ & cho & $\operatorname{chp}$ & $\operatorname{chp}$ \\
\hline chs & cht & cht & cht & cht & cht & cht \\
\hline cht & cht & cif & cipo & cit & cl4 & $\mathrm{clb}$ \\
\hline clbx & cld & $\operatorname{clg}$ & $\mathrm{cml}$ & $\mathrm{cmp}$ & $\mathrm{cmp}$ & $\mathrm{cmr}$ \\
\hline $\mathrm{cms}$ & $\mathrm{cmx}$ & $\mathrm{cnq}$ & cns & cnt & $\operatorname{cod}$ & comicdoc \\
\hline converterx & $\operatorname{cov}$ & $\mathrm{cp}$ & $\mathrm{cpf}$ & $\mathrm{cpf}$ & cpi & cpi \\
\hline cpl & cpp & cpr & $\mathrm{cpt}$ & $\mathrm{cpt}$ & cptx & cpy \\
\hline crf & crp & crwl & $\mathrm{cs}$ & csa & csd & $\operatorname{csd}$ \\
\hline cse & $\operatorname{csf}$ & csk & $\operatorname{csp}$ & cst & $\operatorname{ctd}$ & ctk \\
\hline
\end{tabular}

Continuing next page 


\begin{tabular}{|c|c|c|c|c|c|c|}
\hline ctp & $\operatorname{ctx}$ & cty & $\mathrm{cvj}$ & $\mathrm{cvl}$ & $\mathrm{cvr}$ & $\mathrm{cvt}$ \\
\hline $\mathrm{cw} 3$ & cwk & cwks & cwwp & cxl & $\operatorname{cxp}$ & da \\
\hline da11 & daf & dal & $\mathrm{dbc}$ & $\mathrm{dbi}$ & dbi & $\mathrm{dbm}$ \\
\hline $\mathrm{dbp}$ & dcf & dcf & des & dd & ddc & ddif \\
\hline $\mathrm{ddt}$ & dfl & $\mathrm{dft}$ & $\mathrm{dfv}$ & dgpd & dgr & dgr \\
\hline dgrh & dgs & dhe & dia & dic & dict & disco \\
\hline $\mathrm{dj}$ & $\mathrm{dk} @ \mathrm{p}$ & $\mathrm{dl}$ & dnt & do & $\mathrm{d} ? 4 \mathrm{~d}$ & doc \\
\hline doc & doc & doc & doc & doc & doc & dochtm \\
\hline docm & docmhtml & docx & docxml & dor & dot & dothtml \\
\hline dotm & dotx & dox & dox & dox & dox & $\mathrm{dp}$ \\
\hline $\mathrm{dpd}$ & dpe & dpg & dpgraph & dpo & dproj & dps \\
\hline $\mathrm{dpt}$ & drd & $\mathrm{drf}$ & $\mathrm{drg}$ & $\mathrm{drm}$ & drmx & drt \\
\hline dsf & dsn & dtf & dtp & dtp & dtp & dtr \\
\hline $\mathrm{dtr}$ & $\mathrm{dvb}$ & dvi & $\mathrm{dwz}$ & $\mathrm{dx}$ & $\mathrm{dxd}$ & $\mathrm{dxn}$ \\
\hline dxstudio & dzm & easmx & $\mathrm{eb}$ & ebh & ebkproj & ebs \\
\hline ec4 & ecg & edd & edm & edml & edn & edn \\
\hline edoc & edrwx & edt & efp & efx & egt & ehp \\
\hline emd & eml & emlx & emr & enex & enm & env \\
\hline enx & enyd & epdf & epp & eprtx & ept & epub \\
\hline es & esd & esp & ess & et & ete & eth \\
\hline evo & evt & evt & evy & ewb & ewl & exc \\
\hline exc & $\exp$ & $\mathrm{ez}$ & f96 & fan & faq & far \\
\hline
\end{tabular}

Continuing next page 


\begin{tabular}{|c|c|c|c|c|c|c|}
\hline fax & fbd & $\mathrm{fbl}$ & fbok & fcs & $\mathrm{fd} 2$ & $\mathrm{fdb}$ \\
\hline fdf & $\mathrm{fdm}$ & $\mathrm{fdr}$ & fds & fdt & $\mathrm{fdx}$ & fee \\
\hline ffdata & $\mathrm{fff}$ & $\mathrm{ffs}$ & $\mathrm{fft}$ & $\mathrm{fft}$ & fhz & fig \\
\hline fil & fin & fire & $\mathrm{flb}$ & flg & $\mathrm{flm}$ & flo \\
\hline flo & flp & flp & fls & flw & flw & fly \\
\hline fly & $\mathrm{fm}$ & fmap & fmd & $\mathrm{fmp}$ & fmp3 & $\mathrm{fmt}$ \\
\hline $\mathrm{fmt}$ & $\mathrm{fmt}$ & $\mathrm{fmt}$ & fodp & fodt & $\mathrm{fp}$ & fpage \\
\hline $\mathrm{fpc}$ & fpj & frg & frm & fsd & fsif & ftil \\
\hline $\mathrm{ftl}$ & $\mathrm{ftp}$ & $\mathrm{ftpl}$ & $\mathrm{ftr}$ & $\mathrm{ftr}$ & $\mathrm{fts}$ & fts \\
\hline fwk & fwrt & $f x$ & $f \times 2$ & fxd & fxr & gam \\
\hline gca3 & gca4 & gca4base & gca4party & $\operatorname{gcf}$ & $\mathrm{gcx}$ & gda \\
\hline gdc & gdf & gdoc & gen & gexf & gfc & gform \\
\hline gif2 & gks & gmk & gmp & $\mathrm{gmx}$ & gna & gnd \\
\hline gno & gp1 & gp3 & gp4 & gp5 & gph & gpn \\
\hline gpx & gra & grade & grf & grf & grf & grk \\
\hline grv & grx & gs & gsa & gsc & gsp & gsp \\
\hline $\mathrm{gsw}$ & gtable & gtd & gtp & gui & gwb & gwb \\
\hline h2o & hcr & hcx & hda & hdc & hdt & hed \\
\hline help & hfd & hft & hhp & hhp & hht & his \\
\hline his & hlf & hlp & hlp & hlp & hlp & $\mathrm{hm} 2$ \\
\hline $\mathrm{hm} 3$ & $\mathrm{hmk}$ & $\mathrm{hmp}$ & $h m x$ & hmxp & hmxz & hnc \\
\hline hnd & hot & hpd & hpd & hpj & hpo & hpt \\
\hline
\end{tabular}

Continuing next page 


\begin{tabular}{|c|c|c|c|c|c|c|}
\hline hqz & hsp & hst & hw3 & hw3 & hwp & hxc \\
\hline hxs & hxv & hyp & hype & $i 3 d$ & $\mathrm{i} 3 \mathrm{f}$ & iaf \\
\hline ibatemplate & ibcd & ic & icalevent & icaltodo & icodeproj & icst \\
\hline idc & idml & idx & if & iff & ifo & igx \\
\hline ila & ildoc & $\operatorname{imf}$ & imm & imp & imp & $\mathrm{imr}$ \\
\hline ims & imsp & imv & inct & ind & ind & indb \\
\hline indn & indt & infopathxml & ini & ink & inp & inrs \\
\hline insx & inter & inx & iof & ipf & ipr & ipr \\
\hline iqp & ish1 & it & itp & its & iv-vrml & ivt \\
\hline ivt & iw & iwp & iwprj & iwzip & ix & ix2 \\
\hline ixf & ixi & ixv & jnl & jnt & jpx & jrf \\
\hline jsd & jsd & jtd & jtp & $\mathrm{jtt}$ & jtx & jw \\
\hline jw & jwl & jwrp & kbd & $\mathrm{kcl}$ & $\mathrm{kdc}$ & kdd \\
\hline key & keynote & $\mathrm{kfl}$ & $\mathrm{kfm}$ & kht & kid & $\mathrm{kjv}$ \\
\hline $\mathrm{kmp}$ & knt & $\mathrm{kpr}$ & $\mathrm{kpt}$ & kwd & la & lab \\
\hline latex & lax & $1 \mathrm{~b}$ & lbl & lch & ldf & ldf \\
\hline let & $\operatorname{lgc}$ & $\operatorname{lgf}$ & $\operatorname{lgf}$ & $\operatorname{lgpl}$ & lic & lic \\
\hline lix & 11 & 113 & $\operatorname{lma}$ & $\operatorname{lnt}$ & loc & loc \\
\hline lof & lof & $\operatorname{logonxp}$ & lp2 & lpc & lpd & $\operatorname{lrp}$ \\
\hline 1sd & lsl & lsp & lst & 1st & lst & lth \\
\hline ltx & lwp & lwp & lyr & lyx & $\mathrm{m} ! 93$ & $\mathrm{~m} 11$ \\
\hline $\mathrm{m} 13$ & maca & mag & manu & map & markdn & mars \\
\hline
\end{tabular}

Continuing next page 


\begin{tabular}{|c|c|c|c|c|c|c|}
\hline maw & $\max$ & mbbk & mbd & $\operatorname{mbox}$ & $\mathrm{mbp}$ & $\operatorname{mbx}$ \\
\hline $\mathrm{mc}$ & mcbn & mcc & med & $\mathrm{mcr}$ & mcs & $\operatorname{mcsp}$ \\
\hline $\mathrm{mcw}$ & $\mathrm{mdb}$ & mdbhtml & mdf & mdhtml & $\mathrm{mdk}$ & $\mathrm{mdr}$ \\
\hline me & me & meb & med & mell & mellel & met \\
\hline $\mathrm{mfa}$ & $\mathrm{mfg}$ & mfo & $\mathrm{mfp}$ & $\mathrm{mfp}$ & $\mathrm{mft}$ & mhe \\
\hline mhp & mht & mif & mindnode & mio & mjdoc & mla \\
\hline $\mathrm{mlj}$ & $\mathrm{mlp}$ & $\mathrm{mls}$ & $\mathrm{mm}$ & $\mathrm{mm}$ & mmap & mmas \\
\hline mmat & mmd & $\mathrm{mmf}$ & $\mathrm{mml}$ & mmo & $\mathrm{mmp}$ & $\mathrm{mmpr}$ \\
\hline mmsw & mol & mon & $\mathrm{mp}$ & $\mathrm{mp} 2$ & $\mathrm{mpc}$ & mpj \\
\hline mpls & $\mathrm{mpp}$ & $\mathrm{mpp}$ & $\mathrm{mpp}_{-}$ & $\mathrm{mpr}$ & $\mathrm{mpt}$ & $\mathrm{mpv}$ \\
\hline mpw & mpwd & mpwr & $\operatorname{mpx}$ & mrf & $\mathrm{ms}$ & $\mathrm{ms}$ \\
\hline msd & msdvd & mse & $\mathrm{msf}$ & $\mathrm{msg}$ & mso & $\mathrm{mst}$ \\
\hline $\mathrm{mst}$ & msw & mswd & mswmm & mtp & $\mathrm{mtp}$ & $\mathrm{mtx}$ \\
\hline mtx & $\mathrm{mtx}$ & mug & mvb & $\mathrm{mvt}$ & mvw & $\mathrm{mw}$ \\
\hline $\mathrm{mw}$ & mwd & mwpd & mwpp & mwpr & mwt & $\mathrm{mx} 2$ \\
\hline $\mathrm{mx} 3$ & $\mathrm{nb}$ & $\mathrm{nb}$ & $\mathrm{nb}$ & $\mathrm{nbp}$ & ncb & ncd \\
\hline ncf & nct & ncw & ne3 & nfo & ng & $n j x$ \\
\hline ?nmbtemplate & $\mathrm{nml}$ & not & not & not & note & note \\
\hline $\mathrm{np}$ & npd & $\mathrm{npf}$ & npi & $\mathrm{npl}$ & $\mathrm{npl}$ & npp \\
\hline nrp & ns & nst & nte & nvd & nxd & $n x^{\wedge} \mathrm{d}$ \\
\hline $\mathrm{nx}$ & oa2 & oa3 & oas & obd & obd & obr \\
\hline obx & ocdc & oda & odc & odccubefile & odf & odif \\
\hline
\end{tabular}

Continuing next page 


\begin{tabular}{|c|c|c|c|c|c|c|}
\hline odm & odo & odp & ods & odt & odt\# & ofl \\
\hline ofm & ofm & ofn & oft & ogc & ohw & ole \\
\hline ole2 & olv & oml & omp & omp & one & oos \\
\hline op2 & opd & opd & opj & opj & opn & opt \\
\hline opw & opx & opx & or 3 & osc & otc & otf \\
\hline otg & oth & otl & otp & otp & ott & out \\
\hline ova & ovd & ovs & owm & ows & oxps & oxt \\
\hline $\mathrm{p} 2 \mathrm{bp}$ & $\mathrm{p} 2 \mathrm{~s}$ & p3 & p65 & pac & pad & pag \\
\hline pag & pages & pat & $\mathrm{pb} 1$ & pbd & pbk & pbproj \\
\hline $\mathrm{pc}$ & $\mathrm{pc}$ & pcb & pcr & pcr & $\mathrm{pd}$ & pdf \\
\hline pdfxml & pdf__ & pdf_tsid & pdi & pdl & $\mathrm{pdp}$ & pdp \\
\hline pdt & pez & pfd & pfd & $\mathrm{pfl}$ & $\mathrm{pfp}$ & pgs \\
\hline $\mathrm{ph}$ & phb & $\mathrm{pj} 4$ & pj5 & pjt & pkg & pkp \\
\hline $\mathrm{pl}$ & plb & plf & plg & plp & pls & plx \\
\hline ply & $\mathrm{pm}$ & $\mathrm{pm} 3$ & $\mathrm{pm} 4$ & $\mathrm{pm} 5$ & pm6 & $\mathrm{pm} ?$ \\
\hline pmd & $\mathrm{pml}$ & pmp & $\mathrm{pmt}$ & pmw & $\mathrm{pmx}$ & pod \\
\hline pol & pot & pothtml & potx & ppd & $\mathrm{ppf}$ & ppg \\
\hline ppj & ppl & ppnt & ppot & pps & ppsm & ppsx \\
\hline ppt & ppt3 & ppthtml & pptm & pptmhtml & pptv & pptx \\
\hline pptxml & ppv & $\mathrm{ppv}$ & pr2 & pr3 & pr4 & prc \\
\hline prd & pre & pre & prel & prf & prn & prn \\
\hline prnx & pro4 & project & prproj & prs & prs & prs1 \\
\hline
\end{tabular}

Continuing next page 


\begin{tabular}{|c|c|c|c|c|c|c|}
\hline prs2 & prt & prt1 & prt2 & prv & prx & ps \\
\hline ps 2 & psf & psf & psg & psmd & psn & psr \\
\hline pss & psw & $\mathrm{pt}$ & $\mathrm{pt} 3$ & $\mathrm{pt} 4$ & $\mathrm{pt5}$ & pt6 \\
\hline ptg & ptm & pto & ptx & pub & pub & pub \\
\hline pubf & pubhtml & pubmhtml & pve & pw & pwd & pwd \\
\hline pwi & pwp & pwt & pwt & pwt & $\mathrm{px}$ & pxp \\
\hline $\mathrm{pxt}$ & pxt & pzfx & pzt & $\mathrm{qbl}$ & $\mathrm{gcd}$ & qct \\
\hline qdf & qhep & qhp & qht & qhtm & qprj & qpt \\
\hline grc & qrf & qrt & qu2 & qw & qwd & qwt \\
\hline $\mathrm{qxb}$ & qxt & $\mathrm{rOc}$ & rOf & $\mathrm{rOh}$ & $\mathrm{rOz}$ & $r 3 t$ \\
\hline ra & rap & rav & $\mathrm{rcl}$ & $\mathrm{rcp}$ & rdf & rdf \\
\hline rdf & rdl & rdlx & rec & ref & rels & rep \\
\hline rep & rep & rep & rep & ret & rev & $\mathrm{rf}$ \\
\hline $\mathrm{rft}$ & rgn & rit & rmd & rmd & $\mathrm{rmr}$ & ro \\
\hline roff & rosa & $\mathrm{rpc}$ & rpl & rpmsg & rpn & $\mathrm{rpt}$ \\
\hline rptr & rrd & rrpa & rs & rs & rsf & $\mathrm{rt}$ \\
\hline $\mathrm{rtf}$ & $\mathrm{rtfd}$ & rvc & rvf & rw3 & rxf & $\mathrm{rzb}$ \\
\hline s6bn & s85 & s8bn & sa5 & sam & sam & sbk \\
\hline $\mathrm{sbp}$ & $\mathrm{sbz}$ & sc & sc & scb & scd & scr \\
\hline scriv & scrivx & sct & scw & scw & sd & sdbn \\
\hline sdbz & sdc & sdd & sdd & sdf & $\mathrm{sdg}$ & sdi \\
\hline sdl & $\mathrm{sdm}$ & sdp & sdp & $\mathrm{sdv}$ & sdw & se \\
\hline
\end{tabular}

Continuing next page 


\begin{tabular}{|c|c|c|c|c|c|c|}
\hline se & seek & sem & seo & sff & sff & sff \\
\hline sfs & $\operatorname{sgf}$ & sgl & sgm & sgm & sgm & sgml \\
\hline sgml & sgp & $\operatorname{shb}$ & shb & $\operatorname{shf}$ & shr & shs \\
\hline shw & si & sid & sig & sig & sil & $\operatorname{sim}$ \\
\hline sla & sld3 & sld8 & slds & sle & slf & slf \\
\hline slf & slp & slt & sm & smf & $\mathrm{smf}$ & $\mathrm{smf}$ \\
\hline smh & $\mathrm{smm}$ & smm & smp & smp & sms & snf \\
\hline snf & snp & sod & soi & sox & sp4 & spam \\
\hline spd & spdf & spf & spf & spj & spk & spl \\
\hline spo & spp & spp & spr & sps & sps & sql \\
\hline ss4 & ssc & ssiw & sskd & SSX & st & sta \\
\hline stc & stc & std & std & stg & sti & sti \\
\hline stl & stl & $\mathrm{stm}$ & stm & stp & stp & stw \\
\hline stw & stx & stx & stx & stx & sty & sty \\
\hline sty & $\mathrm{su}$ & sub & sum & SVS & swd & swe \\
\hline swe & swp & sws & sxg & sxg & sxi & sxi \\
\hline sxm & sxm & sxml & SXW & sy3 & $\mathrm{t}$ & $\mathrm{t} 2 \mathrm{k}$ \\
\hline t3001 & t65 & tab & tabula-doc & tah & tal & tbf \\
\hline tcd & tch & tdoc & tds & tef & tex & texi \\
\hline $\operatorname{tg} 1$ & thr & tip & tip & tk & tld & tlt \\
\hline tlx & $\mathrm{tmb}$ & $\mathrm{tmb}$ & tmd & tmd & tmd & $\mathrm{tml}$ \\
\hline tmv & tns & top & topc & tp & tp & tp \\
\hline
\end{tabular}

Continuing next page 


\begin{tabular}{|c|c|c|c|c|c|c|}
\hline tp3 & tpl & tpl & tpl & tpl & tpl & tpl \\
\hline tpo & $\mathrm{tpt}$ & $\operatorname{tpx}$ & $\operatorname{tr} 5$ & tre & tsm & tst \\
\hline tst & tud & tun & tut & tv 4 & tve & twbx \\
\hline tww & txk & txm & txn & txt & txt & txt \\
\hline txt & u98 & udf & udt & ulys & uml & uof \\
\hline uop & uot & updf & uxf & vac & vai & vap \\
\hline $\mathrm{vbd}$ & vbp & vbproj & vcal & vcard & vce & $\operatorname{vcg}$ \\
\hline $\mathrm{vcp}$ & vcproj & vcxproj & vdi & vdoc & vdproj & $\mathrm{vdx}$ \\
\hline vfc & $\mathrm{vh}$ & vhd & vip & $\mathrm{vm}$ & $\mathrm{vmc}$ & vmm \\
\hline $\mathrm{vmr}$ & vmx & vor & vsd & vsp & vst & vsw \\
\hline vsx & vthought & vts & vtx & vup & vxml & $\mathrm{w}$ \\
\hline $\mathrm{w}$ & w51 & w60 & w61 & w6bn & w6w & w8bn \\
\hline w8tn & $w b$ & wbk & wbk & wcl & wcl & wcm \\
\hline wcp & wd0 & wd1 & wd2 & wdbn & wdcd & wdf \\
\hline wdl & wdm & wdoc & ?webtemplate & wgm & wht & whtt \\
\hline wid & wis & wizhtml & wkb & wlf & wlp & wls \\
\hline wmc & wor & word & word & wp & wp4 & wp42 \\
\hline wp5 & wp50 & wp6 & wp7 & wp? & wpa & wpc2 \\
\hline wpd & wpd0 & wpd1 & wpd2 & wpd3 & wpf & wpf \\
\hline wpf & wph & wpl & wpm & wpost & wpostx & wpr \\
\hline wps & wps & wpt & wpw & wpw & wrd & wrf \\
\hline wrg & wri & wrlk & wrt & WS & Ws & ws \\
\hline
\end{tabular}

Continuing next page 


\begin{tabular}{|c|c|c|c|c|c|c|}
\hline ws & ws1 & ws2 & ws3 & ws4 & ws5 & ws6 \\
\hline ws7 & wsa & wsd & wsm & wsq & wsr & wss \\
\hline wt0 & wtbn & wtp & wts & wwcx & wwh & wwk \\
\hline wws & wxmx & wxp & wzn & x40 & x50 & xa0 \\
\hline xap & xav & xbk & XBRL & xdoc & xdp & xdw \\
\hline xe0 & xej & xel & xfdf & xfdl & xft & xgmml \\
\hline xhp & xlc & xlc3 & xlc4 & xlc_ & xlr & xls \\
\hline xlshtml & xlsmhtml & xlthtml & xlw & xmind & xmls & xmmas \\
\hline xmmat & xms & xmt & xpf & xpf & xpr & xpr3 \\
\hline xprj & xps & xsc & xsf & xsn & xtg & xy4 \\
\hline xy4v & xy? & xzfx & yar & ybhtm & ymg & ywp \\
\hline zif & zn & zoi & zpt & zrn & & \\
\hline
\end{tabular}

Table B.5: List of programming source code file extensions allowed through preprocessing whitelist

\begin{tabular}{|c|c|c|c|c|c|c|}
\hline 11 & 19 & $2 c l k$ & $3 r f$ & $4 g e$ & $4 g l$ & 4 th \\
\hline 8 & $8 \times k$ & a & a & a2w & a2x & a51 \\
\hline a66 & a80 & a86 & aas & abap & abc & abl \\
\hline abs & abt & acgi & acm & acr & act & act \\
\hline action & actionscript & actproj & actx & acu & ad & ad \\
\hline ad2 & ada & ?adiumscripts & ads & adt & adx & aep \\
\hline aex & agc & agi & agls & ago & ags & ahk \\
\hline ahtml & aidl & akp & akt & alb & alg & alw \\
\hline alx & aml & amos & amw & anm & ap & ap? \\
\hline
\end{tabular}

Continuing next page 


\begin{tabular}{|c|c|c|c|c|c|c|}
\hline apg & apl & aplt & app & applescript & aps & $\operatorname{armx}$ \\
\hline aro & $\operatorname{arq}$ & art & artproj & ary & as & as3 \\
\hline as? & asax & asbx & asc & asc & asc & ascx \\
\hline asf & ash & asi & asic & asm & asm & $\operatorname{asm} x$ \\
\hline aso & aso & asp & asp & asp+ & asproj & aspx \\
\hline asr & ass & as $\mathrm{x}$ & asz & atl & atomsve & atp \\
\hline atp & au3 & $\mathrm{au} ?$ & aut & avs & awk & awl \\
\hline $\mathrm{axb}$ & axd & axe & axs & $\mathrm{b}$ & $\mathrm{b}$ & b24 \\
\hline $\mathrm{b} 2 \mathrm{~d}$ & bas & bas & bat & $\mathrm{bb}$ & $\mathrm{bbc}$ & bbf \\
\hline bcc & bcf & bcp & bdt & beam & bet & bgm \\
\hline bhs & bi & bil & bin & bks & bli & $\mathrm{bml}$ \\
\hline bml & $\mathrm{bml}$ & bmo & bms & boo & borland & box \\
\hline $\mathrm{bp}$ & bpk & bpo & bpr & bps & bpt & brk \\
\hline brml & brs & brt & brx & bs & bs 2 & bsc \\
\hline bsc & bsh & bsh & bsm & bsv & ?bufferedimage & $\mathrm{bxb}$ \\
\hline$b x 1$ & bxp & bzs & $\mathrm{c}$ & $\mathrm{c}$ & $\mathrm{c}$ & $\mathrm{c}$ \\
\hline c\# & $\mathrm{c}++$ & $\mathrm{c}-$ & $\mathrm{c}-$ & c86 & cal & cap \\
\hline cap & car & cas & $\mathrm{cb}$ & cba & $\mathrm{cbl}$ & $\mathrm{cbp}$ \\
\hline $\mathrm{cbq}$ & cbs & $\mathrm{cc}$ & $\mathrm{cc}$ & $\operatorname{ccs}$ & $\mathrm{cd}$ & cel \\
\hline cfi & cfo & $\operatorname{cfs}$ & $\mathrm{cg}$ & cgi & cgi & cgvp \\
\hline $\operatorname{cg} x$ & $\mathrm{ch}$ & chd & $\mathrm{cl}$ & cla & cla & class \\
\hline $\operatorname{clm}$ & $\operatorname{clp}$ & cls & cls & clss & clu & clw \\
\hline
\end{tabular}

Continuing next page 


\begin{tabular}{|c|c|c|c|c|c|c|}
\hline clw & $\mathrm{cma}$ & cmake & cmd & $\mathrm{cml}$ & $\mathrm{cmm}$ & cmp \\
\hline $\mathrm{cms}$ & cob & $\operatorname{cod}$ & $\operatorname{cod}$ & $\operatorname{cod}$ & $\operatorname{cod}$ & coffee \\
\hline cola & common & con & config & configure & $\cos$ & coverage \\
\hline ?coveragexml & $\mathrm{cp}$ & $\mathrm{cp}$ & $\mathrm{cp}$ & cp? & $\mathrm{cpb}$ & cpp \\
\hline cpr & сpy & cpy & $\mathrm{cpz}$ & $\mathrm{cr}$ & crd & cs \\
\hline $\mathrm{cs}$ & $\mathrm{cs}$ & csattr & $\mathrm{csb}$ & $\csc$ & $\csc$ & $\operatorname{csf}$ \\
\hline csgrad & $\mathrm{csh}$ & $\operatorname{csh}$ & $\operatorname{csh}$ & $\operatorname{csh}$ & cshtml & $\mathrm{csm}$ \\
\hline $\mathrm{csm}$ & $\operatorname{csm}$ & $\operatorname{csp}$ & $\operatorname{csp}$ & csproj & $\operatorname{css}$ & csview \\
\hline $\operatorname{cs} x$ & ctl & ctp & $\mathrm{cx}$ & cxs & cxt & $\operatorname{cxx}$ \\
\hline $\mathrm{c}_{-}$ & d & d & $\mathrm{d} 2 \mathrm{j}$ & $\mathrm{d} 4$ & datasource & $\mathrm{db} 2$ \\
\hline $\mathrm{db} 2 \mathrm{tbl}$ & $\mathrm{db} 2 \mathrm{tr}$ & $\mathrm{db} 2 \mathrm{vw}$ & dba & $\mathrm{dbg}$ & dbheader & $\mathrm{dbml}$ \\
\hline dbo & $\mathrm{dbp}$ & dbpro & dbproj & $\mathrm{dc}$ & dcd & dcf \\
\hline dcp & dct & dd & $\mathrm{ddb}$ & ddp & deb & def \\
\hline def & def & def & def & def & def & defi \\
\hline dep & depend & des & des & des & dev & devpak \\
\hline $\mathrm{dfb}$ & dfd & $\mathrm{dfm}$ & $\mathrm{dfm}$ & dfn & $\mathrm{dg}$ & dgml \\
\hline dht & dhtml & dia & dic & dif & dil & $\mathrm{dkc}$ \\
\hline dlg & dlg & $\mathrm{dmc}$ & $\mathrm{dml}$ & $\mathrm{dml}$ & $\mathrm{dml}$ & $\mathrm{dms}$ \\
\hline do & do & dob & docstates & dor & dot & $\mathrm{dpd}$ \\
\hline dpj & dpk & dpk & dplt & $\mathrm{dpq}$ & $\mathrm{dpr}$ & $\mathrm{dpr}$ \\
\hline dpr & dqy & drc & dro & ds & ds & dsa \\
\hline $\mathrm{dsb}$ & $\mathrm{dsd}$ & dsl & dso & $\mathrm{dsp}$ & dsr & dsym \\
\hline
\end{tabular}

Continuing next page 


\begin{tabular}{|c|c|c|c|c|c|c|}
\hline dsym & $\mathrm{dt}$ & $\mathrm{dtd}$ & dto & dts & dtx & $\mathrm{dvb}$ \\
\hline dwarf & dwp & dws & dwt & dwt & $\mathrm{dxl}$ & $\mathrm{e}$ \\
\hline $\mathrm{e}$ & $\mathrm{e}$ & $\mathrm{e}$ & ebc & ebs & ebs & ebs \\
\hline ebs2 & ebuild & ebx & $\mathrm{ec}$ & ecore & ecorediag & edge \\
\hline edml & egg & el & elc & enml & ens & epj \\
\hline epl & epp & eps2 & epsf & epsi & ept & eql \\
\hline eqn & es & es & esp & ex & exc & exe \\
\hline $\exp$ & $\exp$ & exu & exw & $\mathrm{f}$ & $\mathrm{f}$ & $\mathrm{f} 40$ \\
\hline f77 & f90 & f95 & fasl & fcgi & fdml & fdt \\
\hline $\mathrm{ff}$ & fgl & fil & flm & fmb & fmt & for \\
\hline for & for & $\mathrm{fpc}$ & fpi & fpp & frbd & frj \\
\hline frs & frt & fs & fsi & fsproj & fsproj & fsscript \\
\hline fsx & ftn & fus & fwx & fxl & galaxy & gas \\
\hline gbap & gbl & gc1 & gc3 & gch & generictest & gfe \\
\hline gg & gitignore & gl & glade & gld & glf & glf \\
\hline gls & gml & gml & gnt & goh & gp & $\mathrm{gq}$ \\
\hline gs & gs & gsb & gss & gst & gsym & gus \\
\hline $\mathrm{gv}$ & gyp & $\mathrm{h}$ & $\mathrm{h}++$ & $\mathrm{h}-$ & h16 & h2o \\
\hline h6h & h86 & hal & has & hbx & hbz & hc \\
\hline hew & hh & hic & hkp & hks & hlsl & hms \\
\hline hom & hp? & hpf & hpp & hrh & hs & hsc \\
\hline hsdl & hsm & ht 4 & htc & htd & htm & htr \\
\hline
\end{tabular}

Continuing next page 


\begin{tabular}{|c|c|c|c|c|c|c|}
\hline hxa & hxml & hxp & hxx & hydra & $h_{-}$ & $\mathrm{i}$ \\
\hline $\mathrm{i}$ & iap & iba & ic & ice & icl & icn \\
\hline $\mathrm{idb}$ & $\mathrm{idb}$ & idc & ide & idl & idl & ifp \\
\hline ig & ii & ijs & $\mathrm{ik}$ & il & il & ilk \\
\hline image & $\mathrm{iml}$ & imp & inb & inc & inc & inc \\
\hline inc & inf & ini & ino & inp & ins & ins \\
\hline io & io & $\mathrm{ipb}$ & ipch & ipf & ipp & ipproj \\
\hline ips & iqy & irc & irobo & is & isa & ism \\
\hline iss & iss & isu & isym & ix & $\mathrm{j}$ & jacl \\
\hline jad & jav & java & javajet & $\mathrm{jbc}$ & $\mathrm{jcl}$ & $\mathrm{jcm}$ \\
\hline jdp & $\mathrm{jks}$ & $\mathrm{j} 1$ & jlc & jomproj & jpage & jpd \\
\hline js & js & js & js & jsa & jsb & jse \\
\hline jsf & jsfl & jsh & jsm & json & jsp & jss \\
\hline jsx & jsxinc & judo & $\mathrm{kb}$ & $\mathrm{kcl}$ & kdevprj & ked \\
\hline kex & kix & kmdi & $\mathrm{kml}$ & $\mathrm{kmt}$ & komodo & kon \\
\hline $\mathrm{kpl}$ & ksc & ksh & kst & kumac & 1 & 1 \\
\hline 1 & $11 \mathrm{i}$ & lamp & lap & lasso & lay & lbi \\
\hline lds & lds & less & lex & lex & $\operatorname{lgt}$ & lhs \\
\hline li\$ & lib & $\mathrm{lib}_{-}$ & licx & lisp & lit & 11 \\
\hline 11 & $\operatorname{lml}$ & $\operatorname{lmp}$ & $\operatorname{lmv}$ & $\operatorname{lng}$ & $\operatorname{lng}$ & $\operatorname{lng}$ \\
\hline $\operatorname{lng}$ & $\operatorname{lng}$ & $\operatorname{lng}$ & lnk & $\operatorname{lnp}$ & $\ln x$ & lo \\
\hline loc & lol & lp & lpr & $\operatorname{lpx}$ & $\operatorname{lrf}$ & lrs \\
\hline
\end{tabular}

Continuing next page 


\begin{tabular}{|c|c|c|c|c|c|c|}
\hline ls 1 & lsp & lsp & lss & lst & lua & luca \\
\hline lwa & lxk & 1xsproj & lzco & $\mathrm{m}$ & $\mathrm{m}$ & $\mathrm{m}$ \\
\hline $\mathrm{m}$ & $\mathrm{m} 2$ & $\mathrm{~m} 2 \mathrm{r}$ & $\mathrm{m} 3$ & $\mathrm{~m} 4$ & $\mathrm{~m} 4 \mathrm{x}$ & mac \\
\hline mac & magik & mak & mak & mak & make & make \\
\hline maki & mal & maml & map & mash & master & mat \\
\hline $\max$ & $\mathrm{mb}$ & $\mathrm{mbs}$ & ?mbtemmplate & $\mathrm{mc}$ & $\mathrm{mc}$ & $\mathrm{mc}$ \\
\hline $\mathrm{mcl}$ & $\mathrm{mcm}$ & $\mathrm{mcml}$ & mcp & $\mathrm{mcr}$ & $\mathrm{mcr}$ & $\mathrm{md}$ \\
\hline mdex & mdf & mdf & mdp & mdp & mdp & mec \\
\hline mel & mem & mex & mfcribbon-ms & $\mathrm{mfl}$ & $\mathrm{mg}$ & $\mathrm{mi}$ \\
\hline mingw & mingw32 & mis & $\operatorname{mix}$ & $\mathrm{mk}$ & mke & $\mathrm{ml}$ \\
\hline mli & mli & $\mathrm{mln}$ & $\mathrm{mls}$ & mlsxml & mlts & $\mathrm{mm}$ \\
\hline $\mathrm{mm}$ & $\mathrm{mmb}$ & mmch & mmjs & $\mathrm{mml}$ & mnd & mo \\
\hline moc & $\bmod$ & $\bmod$ & $\bmod$ & moo & $\mathrm{mp} ?$ & $\mathrm{mpd}$ \\
\hline $\mathrm{mpm}$ & mpp & $\operatorname{mpx}$ & mqt & $\mathrm{mrc}$ & mrd & mrl \\
\hline $\mathrm{mrs}$ & $\mathrm{ms}$ & $\mathrm{ms}$ & $\mathrm{msc}$ & mscr & msdev & msha \\
\hline msil & msl & msl & msl & $\mathrm{msm}$ & mso & $\mathrm{msp}$ \\
\hline mss & mss & mst & msve & msym & $\mathrm{mt}$ & mtp \\
\hline $\mathrm{mtx}$ & $\mathrm{mtx}$ & $\mathrm{mv}$ & $\mathrm{mvc}$ & mwp & $\mathrm{mx}$ & mxe \\
\hline mxmf & myapp & mzp & nbin & nbk & ncb & ncx \\
\hline nes & netboot & nlc & $\mathrm{nml}$ & $\mathrm{nms}$ & npi & $\mathrm{nqc}$ \\
\hline nrs & nse & nsi & $\mathrm{nt}$ & $\mathrm{nxc}$ & O & obj \\
\hline obj & obr & obs & ocb & odc & odh & odl \\
\hline
\end{tabular}

Continuing next page 


\begin{tabular}{|c|c|c|c|c|c|c|}
\hline odl & ods & ogl & ogr & ogs & $\operatorname{og} x$ & oks \\
\hline oplm & opt & opx & oqy & orc & osas & osax \\
\hline osg & ow & owd & owl & ox & $\mathrm{p}$ & $\mathrm{p}$ \\
\hline pag & pal & palm & param & pas & pas & pas \\
\hline pas & pas & $\mathrm{pb}$ & pba & pbi & pbl & pbl \\
\hline $\mathrm{pbp}$ & pbq & pbxbtree & pbxproj & $\mathrm{pc}$ & pcd & pch \\
\hline $\mathrm{pcm}$ & pcs & $\mathrm{pd}$ & $\mathrm{pdb}$ & $\mathrm{pdb}$ & $\mathrm{pdb}$ & pde \\
\hline pdl & pdl & pdl & pdo & pdp & pds & pem \\
\hline perl & pf0 & pf1 & pf2 & pf4 & pf? & pfa \\
\hline pfx & pgm & pgm & pgml & $\mathrm{ph}$ & ph & ph3 \\
\hline phl & php & php1 & php2 & php3 & php4 & php5 \\
\hline phps & phs & phtml & pjt & pjt & pjx & $\mathrm{pkb}$ \\
\hline pkg & pkh & $\mathrm{pl}$ & $\mathrm{pl}$ & pl1 & plc & plc \\
\hline plex & pli & plm & pls & plx & plx & $\mathrm{pm}$ \\
\hline $\mathrm{pm}$ & $\mathrm{pmp}$ & pnproj & pnpt & poc & policy & pom \\
\hline $\mathrm{pp}$ & $\mathrm{pp}$ & ppa & ppam & ppo & prg & prg \\
\hline prg & pri & pri & prl & prm & pro & pro \\
\hline proto & prx & psc1 & psd & psf & psl & psl \\
\hline psl & psm1 & psn & pspscript & psu & ptb & ptl \\
\hline ptl & ptx & ptxml & pun & pvs & pwn & pxl \\
\hline $\mathrm{pxl}$ & pxo & pxt & py & py & pyc & pyo \\
\hline pyw & pyx & qcf & qdl & qlc & $\mathrm{qml}$ & qpr \\
\hline
\end{tabular}

Continuing next page 


\begin{tabular}{|c|c|c|c|c|c|c|}
\hline qre & qry & $q x$ & $\mathrm{r}$ & $\mathrm{r}$ & raf & rap \\
\hline rapc & $\mathrm{rb}$ & $\mathrm{rb}$ & $\mathrm{rb}$ & $\mathrm{rb}$ & $\mathrm{rbc}$ & $\mathrm{rbf}$ \\
\hline $\mathrm{rbp}$ & rbs & $\mathrm{rbt}$ & rbw & $\mathrm{rbx}$ & $\mathrm{rc}$ & $\mathrm{rc} 2$ \\
\hline $\mathrm{rcc}$ & $\mathrm{rdf}$ & rdf & rdoff & $\mathrm{rdv}$ & reb & res \\
\hline res & resources & resx & rex & $\operatorname{rexx}$ & $\mathrm{rfs}$ & $\operatorname{rfx}$ \\
\hline rgs & rguninst & $\mathrm{rh}$ & rip & $\mathrm{rlz}$ & $\mathrm{rml}$ & rng \\
\hline rob & robo & robo & rpg & rpj & rptproj & rpy \\
\hline rpyc & rqy & $\mathrm{rrc}$ & $\mathrm{rrh}$ & $\mathrm{rsm}$ & $\mathrm{rsp}$ & rss \\
\hline $\mathrm{rssc}$ & rsym & rts & rul & run & $\mathrm{rvb}$ & rvt \\
\hline rws & rxs & rxs & $\mathrm{s}$ & $\mathrm{s}$ & $\mathrm{s} 2 \mathrm{~s}$ & s43 \\
\hline $\mathrm{s} 4 \mathrm{e}$ & sal & sar & sas & sas & sax & $\mathrm{sb}$ \\
\hline sbi & $\mathrm{sbl}$ & sbr & sbs & sc & sc & sc \\
\hline sca & $\mathrm{scb}$ & $\mathrm{scb}$ & $\mathrm{scm}$ & $\mathrm{scm}$ & $\mathrm{scm}$ & scp \\
\hline scp & scpt & scptd & $\mathrm{scr}$ & scr & $\mathrm{scr}$ & $\operatorname{scs}$ \\
\hline sct & sct & sct & $\operatorname{scx}$ & $\mathrm{scz}$ & sda & sdef \\
\hline sdl & seman & sen & sfx & sh & si & $\operatorname{sim}$ \\
\hline $\operatorname{sim}$ & simple & sit & sjava & sjc & sjs & skp \\
\hline sl & sl & slf & $\sin$ & slt & $\mathrm{sm}$ & $\mathrm{sm}$ \\
\hline sma & smd & sml & sml & $\mathrm{smm}$ & smw & smx \\
\hline snippet & sno & $\mathrm{sp} ?$ & spi & spk & spr & sps \\
\hline spt & spt & spt & $\operatorname{spx}$ & sqb & sql & ?sqldataprovider \\
\hline sqljet & src & src & src & $\operatorname{srp}$ & srz & ss \\
\hline
\end{tabular}

Continuing next page 


\begin{tabular}{|c|c|c|c|c|c|c|}
\hline SS & $\mathrm{ssc}$ & ssc & ssc & ssc & $\operatorname{ssh} 2$ & ssi \\
\hline ssq & st & sti & stl & stm & sts & stx \\
\hline sus & sve & SVX & sw & swg & swt & SXs \\
\hline sxt & sym & sym & sym & $\mathrm{t}$ & $\mathrm{t}$ & $\mathrm{t}$ \\
\hline $\mathrm{t}$ & $\mathrm{t} 2 \mathrm{w}$ & tab & tag & tal & tal & targets \\
\hline tcl & tcl & tcsh & tds & tec & tem & template \\
\hline texinfo & text & $\operatorname{tgml}$ & thtml & ti & tig & tik \\
\hline til & tiprogram & tk & tla & tlc & tld & tlh \\
\hline tlh & tli & tli & tmh & tokend & tpl & tpm \\
\hline tps & tpt & $\operatorname{tpx}$ & tql & tql & tra & triple-s \\
\hline $\operatorname{trs}$ & $\operatorname{trt}$ & tru & tsc & tsq & tst & $\mathrm{ttl}$ \\
\hline tu & tur & turboc 3 & txc & txl & txml & txt \\
\hline $\operatorname{txx}$ & udf & ufdl & ui & uit & uix & ulp \\
\hline umlclass & unx & uvproj & $\mathrm{v}$ & v18 & $\mathrm{v} 4 \mathrm{e}$ & $\mathrm{v} 4 \mathrm{~s}$ \\
\hline vad & vap & $\mathrm{vb}$ & vba & vbe & vbg & vbi \\
\hline vbp & vbproj & vbs & vbw & vbx & $\mathrm{vc1}$ & vc15 \\
\hline $\mathrm{vc} 2$ & $\mathrm{ve} 4$ & $\mathrm{vc5}$ & vc6 & $\mathrm{vc} 7$ & vce & vcp \\
\hline vcproj & vcwin32 & vcxproj & $\mathrm{vd}$ & vddproj & $\mathrm{vdp}$ & vdproj \\
\hline $\mathrm{vgc}$ & vi & vic & vim & vip & viw & vls \\
\hline vmx & vpc & vpi & vps & vre & vrw & vsmacros \\
\hline vspolicy & Vssscc & vstemplate & vtm & vtml & vup & $\mathrm{vx}$ \\
\hline vxml & W & W & waf & was & wax & wbc \\
\hline
\end{tabular}

Continuing next page 


\begin{tabular}{|c|c|c|c|c|c|c|}
\hline wbt & wch & $\mathrm{wcm}$ & wdl & wdx 9 & wfs & win \\
\hline ?win32manifest & wis & wix & wixout & wmc & wml & wml \\
\hline wmlc & wmls & wmlsc & woa & wod & wowproj & wpj \\
\hline wpk & wpm & wpm & Ws & Wsc & wsd & wsdd \\
\hline wsdl & wsf & wsrc & wsym & wX & wxi & wxl \\
\hline wXs & wXs & wZs & $\mathrm{X}$ & $\mathrm{x}$ & $\mathrm{x}$ & xaml \\
\hline xap & xbap & $\mathrm{xbc}$ & $\mathrm{xbd}$ & $\mathrm{xbl}$ & xbn & $\mathrm{xcl}$ \\
\hline xcodeproj & xcp & xdo & $\mathrm{xds}$ & $\mathrm{xfm}$ & $\mathrm{xgl}$ & xhtm \\
\hline xib & xin & $x j b$ & $\mathrm{xl}$ & xla & xlm & $x \operatorname{lm} 3$ \\
\hline$x \operatorname{lm} 4$ & $\mathrm{x} \operatorname{lm}$ & xlv & xmap & xme & $\mathrm{xml}$ & xmljet \\
\hline $\mathrm{xms}$ & xmta & $\mathrm{xn}$ & xoml & $\mathrm{xpl}$ & $\mathrm{xr}$ & $\mathrm{xrc}$ \\
\hline $\mathrm{xsc}$ & $\mathrm{xsc}$ & $\mathrm{Xsc}$ & xsd & xsl & xslt & xsql \\
\hline Xst & $x t x$ & xtxt & $\mathrm{xu}$ & xui & xul & $\mathrm{xwc}$ \\
\hline $\mathrm{y}$ & yab & $\mathrm{yml} 2$ & $\mathrm{yxx}$ & $\mathrm{Z}$ & zasm & zbi \\
\hline zcls & zero & $\mathrm{zfd}$ & zfrm & zfs & zh_tw & $\mathrm{zms}$ \\
\hline zpk & zpl & $\mathrm{zsc}$ & zsh & zsrc & zts & zWs \\
\hline$\sim 1 \sim$ & df & $\sim \mathrm{pa}$ & & & & \\
\hline
\end{tabular}




\begin{tabular}{|c|c|} 
Dataset & Number of Records \\
\hline Set 1 & 567 \\
\hline Set 2 & 193 \\
\hline Set 3 & 124 \\
\hline Set 4 & 528 \\
\hline Set 5 & 1073 \\
\hline Set 6 & 1041 \\
\hline Set 7 & 973 \\
\hline Set 8 & 1153 \\
\hline Set 9 & 1046 \\
\hline Set 10 & 1097 \\
\hline
\end{tabular}

Table B.6: Record counts across datasets 


\section{Bibliography}

[1] The source for fire extensions information. Website, 2012. http://www.file-extensions. org/.

[2] Barman Badan. Knol: a gateway to encyclopedic article. http://badanbarmanknol.wordpress.com/article/knol-a-gateway-to-encyclopedic-article3bexxvrdm2i7n-13/, March 2010.

[3] M. Baez, A. Birukou, F. Casati, and M. Marchese. Addressing information overload in the scientific community. Internet Computing, IEEE, 14(6):31 -38, nov.-dec. 2010.

[4] Liliana Cabral, John Domingue, Enrico Motta, Terry Payne, and Farshad Hakimpour. Approaches to semantic web services: An overview and comparisons. pages 225-239, 2004.

[5] Guanling Chen and David Kotz. A Survey of Context-Aware Mobile Computing Research. Technical report, Hanover, NH, USA, 2000.

[6] Harry Chen, Tim Finin, and Anupam Joshi. An ontology for context-aware pervasive computing environments. Special Issue on Ontologies for Distributed Systems, Knowledge Engineering Review, 18:197-207, 2003.

[7] Scott Deerwester, Susan T. Dumais, George W. Furnas, Thomas K. Landauer, and Richard Harshman. Indexing by latent semantic analysis. Journal of the American Society for Information Science, 41:391-407, 1990.

[8] Jacob Eisenstein and Randall Davis. Visual and linguistic information in gesture classification. In ACM SIGGRAPH 2007 courses, SIGGRAPH '07, New York, NY, USA, 2007. ACM.

[9] L. Fisher. How to Dunk a Doughnut: The Science Of Everyday Life. Arcade Pub., 2003.

[10] Eibe Frank, Mark Hall, and Bernhard Pfahringer. Locally weighted naive bayes. In Proceedings of the Conference on Uncertainty in Artificial Intelligence, pages 249-256. Morgan Kaufmann, 2003.

[11] Gregory Gay, Tim Menzies, Bojan Cukic, and Burak Turhan. How to build repeatable experiments. In Proceedings of the 5th International Conference on Predictor Models in Software Engineering, PROMISE '09, pages 15:1-15:9, New York, NY, USA, 2009. ACM. 
[12] Paolo Giudici. Applied Data Mining: Statistical Methods for Business and Industry (Statistics in Practice). Wiley, 2003.

[13] Thomas R. Gruber. Toward principles for the design of ontologies used for knowledge sharing. Int. J. Hum.-Comput. Stud., 43(5-6):907-928, December 1995.

[14] Mark Hall, Eibe Frank, Geoffrey Holmes, Bernhard Pfahringer, Peter Reutemann, and Ian H. Witten. The weka data mining software: an update. SIGKDD Explor. Newsl., 11(1):10-18, November 2009.

[15] Robert C. Holte. Very simple classification rules perform well on most commonly used datasets. In Machine Learning, pages 63-91, 1993.

[16] Xin Jin, Yanzan Zhou, and Bamshad Mobasher. Web usage mining based on probabilistic latent semantic analysis. In Proceedings of the tenth ACM SIGKDD international conference on Knowledge discovery and data mining, KDD '04, pages 197-205, New York, NY, USA, 2004. ACM.

[17] James Joyce. Bayestheorem. In Edward N. Zalta, editor, The Stanford Encyclopedia of Philosophy. Fall 2008 edition, 2008.

[18] M.W. Kadous, Mohammed Waleed Kadous, and Supervisor Claude Sammut. Temporal classification: Extending the classification paradigm to multivariate time series. Technical report, University of New South Wales, 2002.

[19] Marziah Karch. Annotum definition. Website, 2012. http://google.about.com/od/ experiment_graveyard/g/Annotum-Definition.htm/.

[20] Bryan Lemon. The effect of locality based learning on software defect prediction . ProQuest, UMI Dissertation Publishing, 2011.

[21] Bryan Lemon and Daniel Sloan. Graphical knowledge advantage machine. 2012.

[22] Henry Lieberman. Letizia: an agent that assists web browsing. In Proceedings of the 14th international joint conference on Artificial intelligence - Volume 1, IJCAI'95, pages 924-929, San Francisco, CA, USA, 1995. Morgan Kaufmann Publishers Inc.

[23] Pamela J. Ludford, Dan Frankowski, Ken Reily, Kurt Wilms, and Loren Terveen. Because i carry my cell phone anyway: functional location-based reminder applications. In Proceedings of the SIGCHI conference on Human Factors in computing systems, CHI '06, pages 889-898, New York, NY, USA, 2006. ACM.

[24] J. B. MacQueen. Some methods for classification and analysis of multivariate observations. In L. M. Le Cam and J. Neyman, editors, Proc. of the fifth Berkeley Symposium on Mathematical Statistics and Probability, volume 1, pages 281-297. University of California Press, 1967. 
[25] Sharon Bertsch McGrayne. The Theory That Would Not Die: How Bayes' Rule Cracked the Enigma Code, Hunted Down Russian Submarines, and Emerged Triumphant from Two Centuries of Controversy. Yale University Press, 2011.

[26] Hiroshi Motoda and Kenichi Yoshida. Machine learning techniques to make computers easier to use, 1998.

[27] Tapio Niemi, Marko Niinimäki, Jyrki Nummenmaa, and Peter Thanisch. Constructing an olap cube from distributed $\mathrm{xml}$ data. In Proceedings of the 5th ACM international workshop on Data Warehousing and OLAP, DOLAP '02, pages 22-27, New York, NY, USA, 2002. ACM.

[28] Martin L. Puterman. Markov Decision Processes: Discrete Stochastic Dynamic Programming. John Wiley \& Sons, Inc., New York, NY, USA, 1st edition, 1994.

[29] J. Ross Quinlan. C4.5: Programs for Machine Learning (Morgan Kaufmann Series in Machine Learning). Morgan Kaufmann, 1992.

[30] R. Reddy, L. Wang, S. Reddy, S. Devalapalli, G. Sasanka, S. Macha, S. Teja, R. Doppalapudi, J. Yu, and J. Yu. Vijjana: A pragmatic model for collaborative, self-organizing, domain centric knowledge networks. In IKE, pages 116-121, 2008.

[31] Irina Rish. An empirical study of the naive Bayes classifier. In IJCAI-01 workshop on "Empirical Methods in AI".

[32] Chris Rorden. ezanova free statistical software. Website, 2012. http://www. mccauslandcenter.sc.edu/mricro/ezanova/index.html.

[33] Mehran Sahami, Susan Dumais, David Heckerman, and Eric Horvitz. A bayesian approach to filtering junk e-mail, 1998.

[34] Leo Sauermann, Gunnar Aastr, Malte Kiesel, Heiko Maus, Dominik Heim, Danish Nadeem, Benjamin Horak, and Andreas Dengel. A.: Semantic desktop 2.0: The gnowsis experience. In International Semantic Web Conference. Volume 4273 of Lecture Notes in Computer Science, pages 887-900. Springer, 2006.

[35] Leo Sauermann, Ansgar Bernardi, and Andreas Dengel. Overview and outlook on the semantic desktop. In In Proc. of Semantic Desktop Workshop at the ISWC, 2005.

[36] Elizabeth Shriver, Christopher Small, and Keith A. Smith. Why does file system prefetching work? In Proceedings of the annual conference on USENIX Annual Technical Conference, ATEC '99, pages 6-6, Berkeley, CA, USA, 1999. USENIX Association.

[37] Randy Franklin Smith. The Windows Server 2003 Security Log Revealed. Monterey Technology Group, Incorporated, USA, 2nd edition, 2007. 
[38] Jaideep Srivastava, Robert Cooley, Mukund Deshpande, and Pang-Ning Tan. Web usage mining: discovery and applications of usage patterns from web data. SIGKDD Explor. Newsl., 1(2):12-23, January 2000.

[39] Werner Vogels. File system usage in windows nt 4.0. SIGOPS Oper. Syst. Rev., 33(5):93-109, December 1999.

[40] Luyi Wang, Ramana Reddy, Sumitra Reddy, and Asesh Das. A context centric model for building a knowledge advantage machine based on personal ontology patterns. In $S W W S$, pages 99-105, 2011.

[41] Geoffrey I. Webb, Janice R. Boughton, and Zhihai Wang. Not so naive bayes: Aggregating one-dependence estimators. Machine Learning, 58:5-24, 2005. 10.1007/s10994-005-4258-6.

[42] Ian H. Witten and Eibe Frank. Data Mining, Second Edition: Practical Machine Learning Tools and Techniques, Second Edition (The Morgan Kaufmann Series in Data Management Systems). Morgan Kaufmann, 2005.

[43] Wolfgang Woerndl and Georg Groh. A social item filtering approach for a mobile semantic desktop application. In AAAI Spring Symposium: Social Semantic Web: Where Web 2.0 Meets Web 3.0, pages 82-83, 2009.

[44] Harry Zhang. The Optimality of Naive Bayes. In Valerie Barr and Zdravko Markov, editors, FLAIRS Conference. AAAI Press, 2004.

[45] Hai Zhuge. Autonomous semantic link networking model for the knowledge grid. Concurrency and Computation: Practice and Experience, 19(7):1065-1085, 2007. 